Rhode Island College

Digital Commons @ RIC

\title{
A Phenomenological Exploration of How Public School Administrators Balance Teacher Evaluation and Support of Teacher Growth
}

\author{
Mary F. Slattery \\ Rhode Island College
}

Follow this and additional works at: https://digitalcommons.ric.edu/etd

Part of the Educational Assessment, Evaluation, and Research Commons, and the Other Educational Administration and Supervision Commons

\section{Recommended Citation}

Slattery, Mary F., "A Phenomenological Exploration of How Public School Administrators Balance Teacher Evaluation and Support of Teacher Growth" (2017). Master's Theses, Dissertations, Graduate Research and Major Papers Overview. 176.

https://digitalcommons.ric.edu/etd/176

This Thesis is brought to you for free and open access by the Master's Theses, Dissertations, Graduate Research and Major Papers at Digital Commons @ RIC. It has been accepted for inclusion in Master's Theses, Dissertations, Graduate Research and Major Papers Overview by an authorized administrator of Digital Commons @ RIC. For more information, please contact digitalcommons@ric.edu. 


\section{A PHENOMENOLOGICAL EXPLORATION}

OF HOW PUBLIC SCHOOL ADMINISTRATORS

BALANCE TEACHER EVALUATION

AND SUPPORT OF TEACHER GROWTH

BY

MARY F. SLATTERY

\section{A DISSERTATION SUBMITTED IN PARTIAL FULFILLMENT OF THE REQUIREMENTS OF DEGREE OF DOCTOR OF PHILOSOPHY OF EDUCATION}

UNIVERSITY OF RHODE ISLAND

AND

RHODE ISLAND COLLEGE

2017 
DOCTOR OF PHILOSOPHY DISSERTATION

\author{
OF \\ Mary Frances Slattery
}

\title{
APPROVED:
}

Dissertation committee:

Major professor:

Theresa Deeney

Committee:

Kathy Peno

Karen Castagno

John Niska

RIC: Donald Halquist

Dean, Feinstein School of Education - RIC

URI: $\quad$ Nasser H. Zawia

Dean of the Graduate School - URI

UNIVERSITY OF RHODE ISLAND

AND

RHODE ISLAND COLLEGE 


\begin{abstract}
Educational reform efforts over the past several decades have focused on different ways to address student achievement. Since the quality of the teacher that students have is one of the biggest in-school factors that impact student achievement, schools have focused on identifying, measuring, and improving teacher quality. Some of those reform efforts have focused their attention on teacher evaluation as a way of increasing student achievement. There has been much research on the components quality evaluation tools. There is also research on ways to impact and change teacher practice. Unfortunately most evaluation tools do not impact teaching practice. Rhode Island administrators are current facing the challenge of implementing a high stakes evaluation system while also finding ways to help support growth and development of their teachers. This phenomenological study used open-ended interviews to understand how six administrators in Rhode Island negotiate that complexity. Administrators interviewed emphasized the importance of developing a trusting positive climate and utilizing this climate, along with various components of the evaluation system, to provide teachers with the kinds of support that will impact teacher growth and practice in the classroom. Administrators also expressed their frustration at some of the elements of the evaluation system and limitations they have found in their ability to impact teacher change. Findings from this study have implications for those revising or creating educator evaluation systems, as well as for administrators who must use high stakes evaluation systems while simultaneously attempting to impact teacher growth, development and change in practice.
\end{abstract}




\section{ACKNOWLEDGEMENTS}

To my major professor, Theresa Deeney, it's been a long road and I'm so happy to have travelled it with you. Thank you for your faith in me and believing that I could do it even when I sometimes doubted myself. Thank you for reading all of my drafts and talking me through all of my ideas. I want to thank you not only for your professional help and support, but also for your personal concern and friendship. Not only did you guide me along this journey answering my questions and pushing my thinking, you also took the time to listen to me and support me in the loss of my father. You understood the importance of completing this process for me.

To my committee members, Kathy Peno, Karen Castagno, Janet Johnson, and John Niska. Thank you for all of your time thoughtfully reading my drafts even when you were on sabbatical, out of the state or even out of the country. Your questions and suggestions helped shaped my thinking and my final product here today.

To all of the administrators who took the time to meet with me and talk about their challenges and their successes. The best part of this process for me was talking to other administrators who want to do the right thing for their teachers and who are willing to keep on trying given any and every new initiative that they face. Thank you for taking the time to share your thoughts with me. I was inspired by your caring, your love for your job, and your willingness to take time out of your already overbooked schedules to sit and talk with me about our shared passion of supporting teachers.

To Fern Reisinger, who read every page of every draft of every part of this dissertation, many of which never even made it into the final copy. Thank you for 
believing in me and for constantly encouraging me to keep writing. You are just the right balance between boot and wind. Your encouragement and belief in me never waivered.

To all of my teachers who are my inspiration behind this. I wanted to figure out how I could be a better principal in supporting you. Throughout the past seven years you have had the "benefit" of all of the research I have been doing and you have endured my many attempts to improve professional development and support for you at school. Hopefully, together, we have made the evaluation something that works for all of us and helps us all grow.

To my family, friends, colleagues, teachers, my secretary, students, parents, cohort members, and school and district administrators, thank you for your support and encouragement throughout this entire process. 


\section{DEDICATION}

This dissertation is dedicated to God. Each morning as I prayed for your guidance, you gave it to me. You were able to keep me on track and moving in the right direction for this dissertation to be completed. When I follow your lead, it always turns out for the best.

This dissertation is also dedicated to my dad, Francis Slattery, who passed away on October 31, 2016. I know you are proud of me for doing this. I wish you were here with me to see me finish it, but you are always here with me in my heart.

To my mama. You have always been my biggest supporter and fan. I am so happy I have completed this and become the first Dr. Slattery in the family.

To my sons, Christian and Jes. Thank you for understanding how important this is to me. You are my inspiration behind all I do. I want you to know that you should always pursue your dreams; even when they are hard and take a lot of hard work and effort, you should always keep on working. I am always here cheering you on.

To my sister Maureen. It's been a rough year but I'm so glad that I always have you to understand and help me through everything. Your love and support has meant more than I can express.

To all of my family. My sister and brothers, in-laws, nieces and nephews, great nephew, cousins, aunts and uncles, and grandparents. It was Grandpa Rischitelli who taught us all the importance of education and pursuing your dreams through education.

To my besties, Kathy and Julie. Thank you for always cheering me on and supporting me through everything. Your faith in me has helped me to fly in so many different ways. 
To Andy. You make me want to me a better principal so I can inspire you and help you grow as a teacher. Thank you for taking the time to listen to my ideas, give me your feedback, and even conduct an interview with me. There is no one I would rather be interviewed by. In addition to that you are my best friend and my voice of reason in the chaos. Thanks for always being there and knowing when to talk and when to just listen. Thanks also for letting me use your house as my retreat. 


\section{TABLE OF CONTENTS}

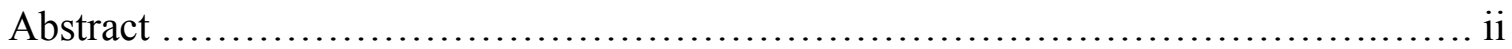

Acknowledgements .................................................... iii

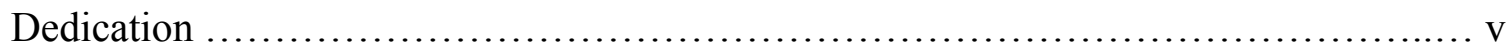

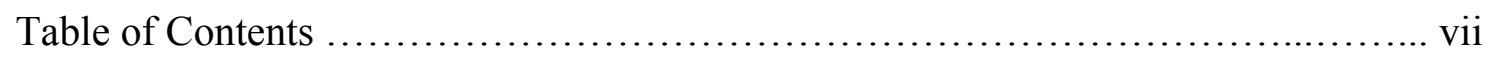

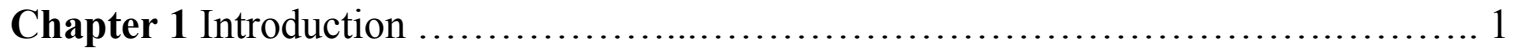

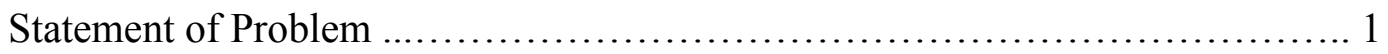

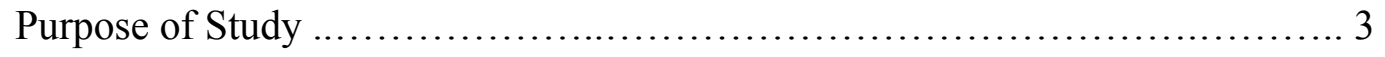

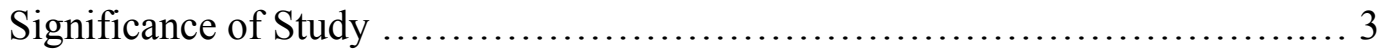

Current Reform Context ............................................ 4

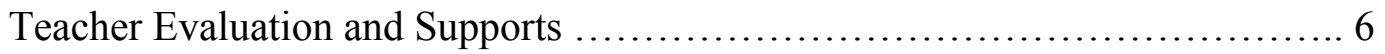

Research Questions ............................................... 10

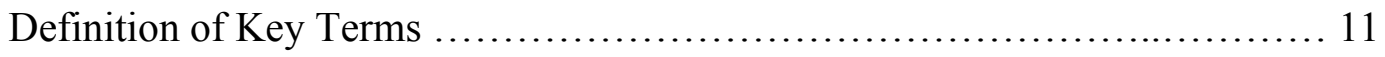

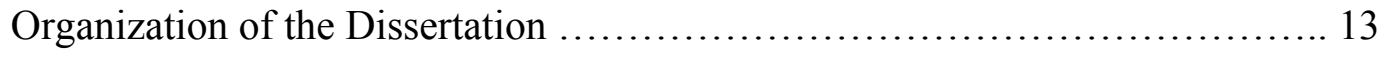

Chapter 2 Theoretical Frame and Literature Review ............................ 14

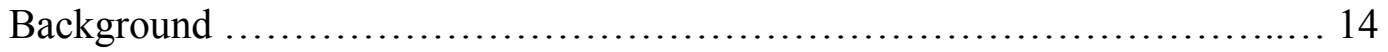

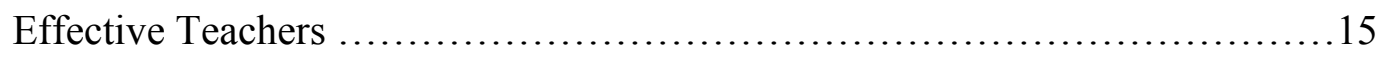

Measuring Teacher Effectiveness ..................................... 16

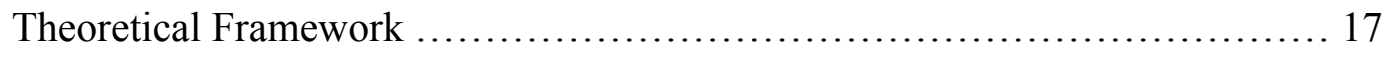

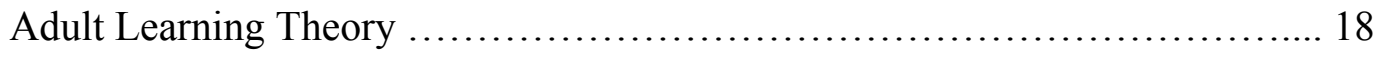

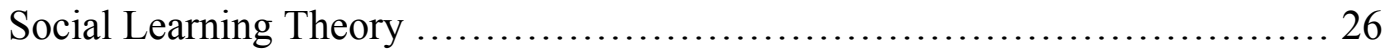

Implications of Theory and Research Supporting Teacher Growth ............ 29 
Implications of Theory and Research on Effective Teacher Professional Development ................................................... 32

Administrators' Role in Professional Development and Evaluation .............. 36

Deficiencies in the Literature ........................................... 38

Chapter 3: Methodology ......................................................... 40

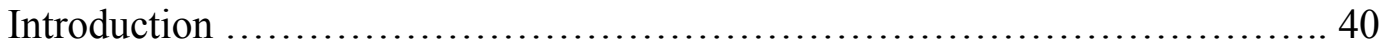

Research Questions ................................................... 41

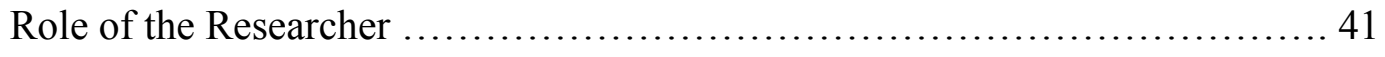

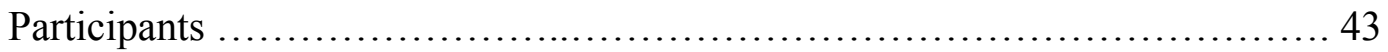

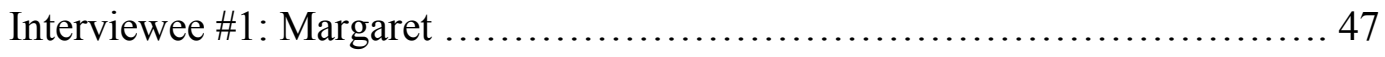

Interviewees \#2 \& \#3: Becky \& Thomas .................................. 50

Interviewee \#4: Alicia ................................................ 52

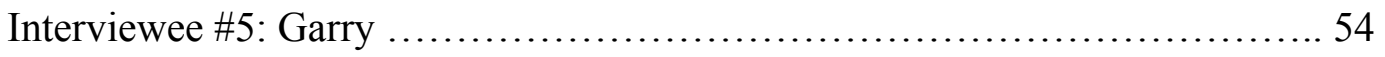

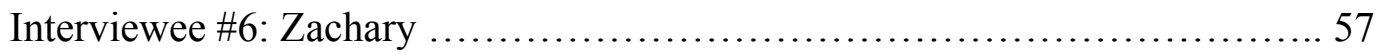

Data Collection, Sources, and Timetable .................................. 60

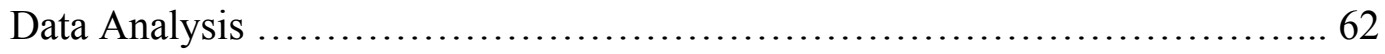

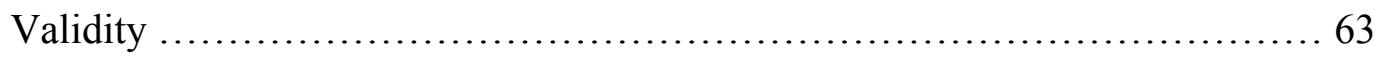

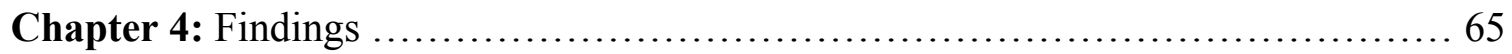

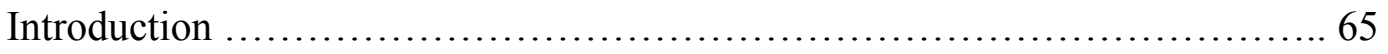

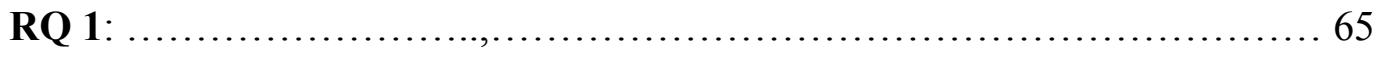

Strategic Use of Professional Development .................................. 66

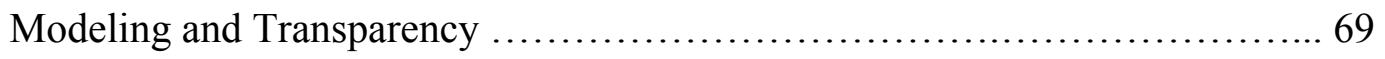

Differentiation and Personalization ...................................... 71 
Social Learning Supports ............................................. 75

Supports for New Teachers ............................................ 76

Support for Teachers Needing More Support .................................... 79

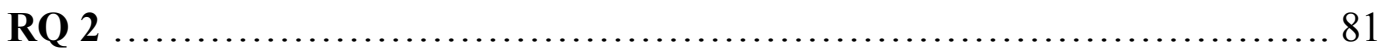

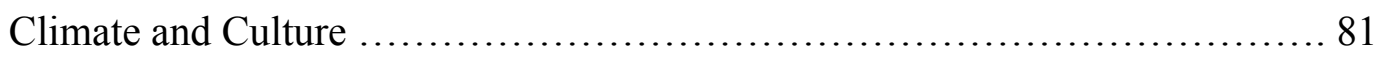

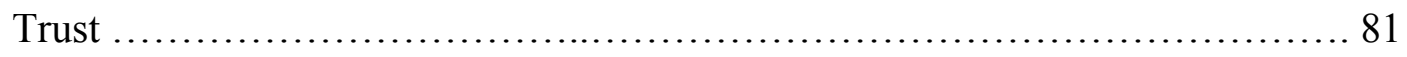

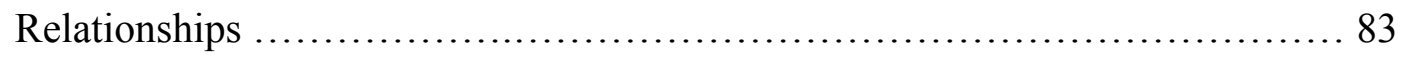

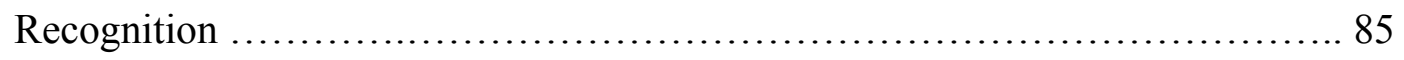

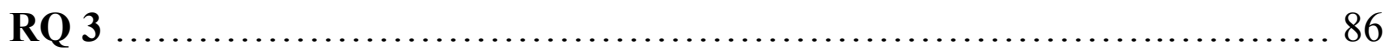

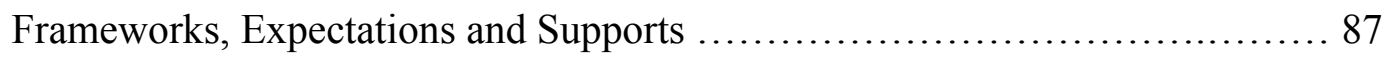

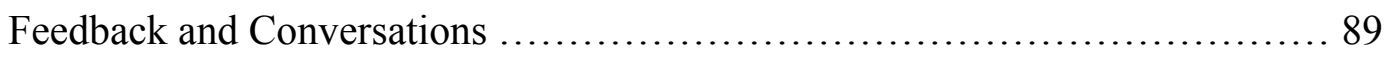

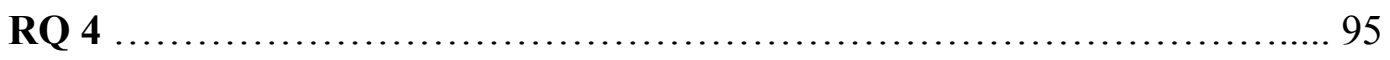

Accountability - No Teeth …........................................... 96

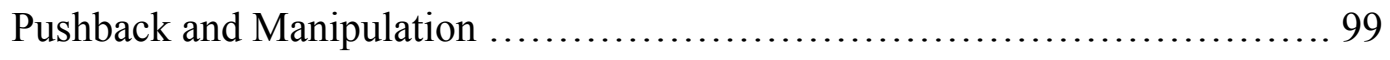

Negativity about the Evaluation ......................................... 101

Time, Paperwork, Limitations ........................................... 103

Restriction of the Evaluation .......................................... 107

Balancing Supports and Evaluation ......................................... 109

Reducing Stress Associated with Evaluation ................................ 109

Alternative Approaches to Accountability ................................ 114

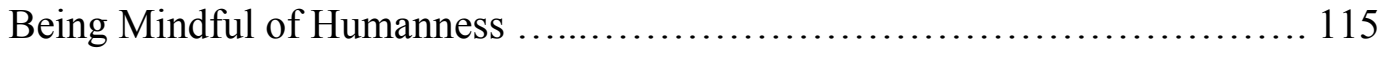

Merging Support and Evaluation ............................................. 119

Recommendation to Selves ............................................ 122 
Chapter Summary

Chapter 5: Discussion

Introduction

Summary of Qualitative Findings 124

Challenge \#1: Manipulation of Data 125

Solution \#1: Social Learning and Peer Accountability 127

Challenge \#2: One Size Does Not Fit All 129

Solution \#2(a): Differentiation 130

Solution \#2(b): Tiered Evaluation 131

Challenge \#3: Negativity Toward Evaluation

Solution \#3: Positive Culture and Building Trust 134

Limitations of the Study 136

Implications 136

Suggestions for Future Research 138

\section{Appendices}

Appendix A: Participant Email 139

Appendix B: Participant Questionnaire 141

Appendix C: Informed Consent Form 143

Appendix D: Interview Protocol 145

Bibliography 148 


\section{List of Tables}

TABLES

PAGE

Table 1.1 Comparison of Evaluation and Supervision Practices $\ldots \ldots \ldots \ldots \ldots \ldots \ldots \ldots \ldots$

Table 2.1 Adult Development and Epistemological Thinking $\ldots \ldots \ldots \ldots \ldots \ldots \ldots \ldots \ldots . \ldots . \ldots \ldots$

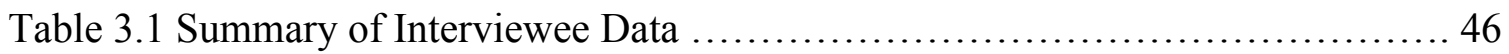




\section{Chapter 1: Introduction}

\section{Statement of the Problem}

The goal of federal and state educational reform efforts over the past several decades has been ensuring academic success for all students. Given that many students do not consistently meet expectations in core subject areas (National Assessment of Educational Progress [NAEP], 2014), targeting student success is a laudable goal. Many reform efforts have framed the issue of improving student achievement through a curriculum lens. In other words, improving curriculum will help to improve student achievement. For example, No Child Left Behind (U. S. Department of Education, 2001) sought to improve student achievement by setting challenging academic standards and encouraging the use of "research-based" instructional practices. Evaluative systems designed to assess the effectiveness of these program are often linked to reform efforts. In the case of curriculum reform, effectiveness was measured both by student achievement on state and national assessments, and by the insurance of instructional fidelity tied to continued state and federal funding.

Current federal reform efforts continue to emphasize student achievement with various measures designed to demonstrate student success. Yet the lens used to frame the problem of student achievement has shifted from curriculum to teachers. Race to the Top (U.S. Department of Education, 2013), one of the more recent federal reform initiatives, has highlighted teacher effectiveness as a vehicle for improving student achievement, as student achievement has been linked to teacher quality (Brophy, 1986; DarlingHammond, 2000; Rivkin, Hanushek, \& Kain, 2005; Wright, Horn, \& Sanders, 1997). Federal funding was made available to states that complied with 
recommendations outlined in Race to the Top. Because of its focus on teacher quality, Race to the Top mandated measures of teacher effectiveness in addition to student achievement.

Evaluation systems are at the center of measuring and identifying teacher effectiveness. The rationale behind this process is that teachers who demonstrate effectiveness on specific measures will, ultimately, produce students who achieve at higher standards academically. Yet, even when employing best practices in measuring teacher effectiveness, teacher evaluation systems have not been shown to impact teaching practices (Donaldson, 2013; Hazi \& Rucinski, 2009; Marshall, 2012; Murphy, Hallinger, \& Heck, 2013; Starratt, 1992). This may lay in the design and implementation of teacher evaluation systems. Evaluations are used to identify effective or ineffective teaching per designated criteria, but are not designed to assist teachers identified as less than effective in improving their teaching and classroom practices (Wise, Darling-Hammond, McLaughlin, \& Bernstein, 1985). Therefore, the responsibility of identifying ways to support teachers' growth in professional practice falls largely to school administrators. Administrators then serve the dual role of evaluating teachers while also supporting them in improving their practice (Berube \& Dexter, 2006; Donaldson, 2013).

Recent studies (Donaldson, 2013; Hazi \& Rucinski, 2009; Murphy et al., 2013; Starratt, 1992) have identified a conflict in the role of the administrator as the individual who is responsible for both evaluating and supporting teachers. Yet no research exists discussing how administrators manage these dual roles, and fulfill obligations of both evaluation and support or supervision. 


\section{Purpose of the study}

The purpose of this qualitative phenomenological study is to investigate how current Rhode Island (RI) administrators manage the dual roles of supporting and encouraging teacher growth, while also being responsible for evaluating teachers using a current high stakes teacher evaluation system. I chose phenomenology as the research method because it focuses on the essence of a particular experience, or phenomenon, as described by the participants (Creswell, 2009; Fraenkel, Wallen, \& Hyun, 1993). The phenomenon I am studying is implementing the Rhode Island educator evaluation system while also supporting teacher growth as a RI administrator. By using phenomenological interviews and collecting artifacts from administrators, I will be looking at the actual lived experiences of administrators and what they are doing to try to maintain a balance between evaluation and support.

\section{Significance of Study}

Although federal educational reform efforts have attempted to address the issue of student achievement in a variety of ways, many have focused, at least in part, on teacher quality. A Nation at Risk (National Commission on Excellence in Education, 1983) recognized the importance of good teachers and suggested connecting teaching salaries, as well as promotion and retention decisions to teacher evaluation. No Child Left Behind (U. S. Department of Education, 2001) also stressed the significance of "highly qualified" teachers. A recent reform effort, Race to the Top (RTTT), focuses on having an effective teacher in every classroom through developing teacher evaluation systems, and revising compensation and retention policies to identify, promote and reward teacher effectiveness (U.S. Department of Education, 2013). 
With the increased attention given to teacher evaluation and measuring teacher effectiveness, there has been renewed research on best practices related to evaluation systems and measuring and identifying teacher effectiveness (Darling-Hammond, Amrein-Beardsley, Haertel, \& Rothstein, 2012; Goe, Bell, \& Little, 2008). Comprehensive evaluation systems consisting of multiple measures, and including training and education for both teachers and administrators have been shown to be the most widely accepted and effective evaluation systems (Darling-Hammond et al., 2012; Goe et al., 2008; Murphy et al., 2013).

\section{Current Reform Context}

In 2011, the Rhode Island Department of Education (RIDE) applied for and received Race to the Top (RTTT) grant funding. Part of the grant application required RIDE to create and implement a statewide teacher evaluation system (Rhode Island Department of Education, 2010). The RIDE model utilized many research-based best practices in teacher evaluation to create a multifaceted evaluation tool, and provided both teachers and administrators multiple opportunities for training and feedback (Rhode Island Department of Education, 2012).

One of the components of the RIDE evaluation system is Student Learning Objectives or SLOs. SLOs are designed to measure teacher impact on student academic achievement through the setting of long-term, measureable, academic goals. Teachers set at least two and no more than four SLOs at the beginning of the year and monitor them throughout the year. Part of the final effectiveness rating at the end of the year is based on student's achievement of these goals. Administrators approve teacher SLOs and assign a score of Not Met, Nearly Met, Met or Exceeded at the end of the year (RIDE, 2012). 
Teachers also set Professional Growth Goals (PGGs) each year they are evaluated designed to measure teacher's professional growth during the year. PGGs may be set by the district, school, or individual teacher. At the end of the year a final effectiveness rating is determined by combining a teacher's scores on their SLOs, observations scores including both a classroom environment score and an instruction score, as well as a professional responsibilities score which includes a score for the PGG. Observations are scored on a 4-point rubric with 1 being the lowest and 4 being the highest. Final effectiveness ratings range from Highly Effective, Effective, Developing to Ineffective. Teachers will be placed on an evaluation cycle based on their rating the previous year. Highly effective teachers are evaluated once every three years. Effective teachers are evaluated once every two years. Teachers rated developing or ineffective will be evaluated annually and will also be placed on a performance improvement plan. Improvement plans are designed to provide support to those teachers in need. RIDE has also created the Educator Performance and Support System (EPSS), which is an online program to aide in the collection, sharing and managing of all evaluation related data and documents (RIDE 2012).

Seeking to recruit and develop competent educators, simultaneous to unveiling the educator evaluation system, RIDE developed an induction coaching program which provided novice teachers with an outside trained coach to support them in their induction year (Rhode Island Department of Education, 2010). This regular support from an experienced and trained induction coach was intended to scaffold beginning teachers in moving along the developmental continuum from novice to expert. Due to lack of sustainable funding after the RTTT grant expiration, the induction-coaching program was 
discontinued, but the evaluation system remains in effect for all teachers. The continuation of support for beginning and more veteran teachers, including those identified as in need of an improvement plan, has fallen to the discretion of individual districts and/or schools. As of now, there is no data measuring whether the RI educator evaluation process has produced improvement in teacher quality.

\section{Teacher Evaluation and Supervision}

Several studies show that evaluating teachers does not lead to changes in teaching practices in the classroom (Berube \& Dexter, 2006; Campbell, Kyriakides, \& Muijs, 2003; Donaldson, 2013; Starratt, 1992; Wise, et al., 1985). In a recent national survey (Donaldson, 2013), only 26\% of teachers felt that teacher evaluation was useful or effective for them. Although $83 \%$ of principals reported that "monitoring teachers' work in the classroom was a helpful practice for improving their instruction" (p. 844), only $37 \%$ of teachers found this same practice helpful.

The lack of impact that evaluation has on teaching practices may be due to differences between evaluation and supervision practices. Table 1.1 summarizes this research.

\section{Table 1.1}

Comparison of Evaluation and Supervision Practices

\begin{tabular}{ll}
\hline Evaluation & Supervision \\
\hline & Supportive \\
Rate or judge effectiveness & Provide assistance \\
Accountability & Formative assessment \\
Summative ratings & Personal development \\
Inform personnel decisions & Individual and whole school need \\
& identification \\
\hline
\end{tabular}

Note: Information complied and adapted from Berube and Dexter (2006), Donaldson (2013) and Starratt (1992) 
Evaluation practices tend to rate or judge a teacher's effectiveness in the classroom (Berube \& Dexter, 2006). They are more often focused on accountability, summative ratings, and removal of ineffective teachers than on teacher growth and development. They tend to serve an organizational purpose by informing personnel decisions and holding teachers accountable for individual student growth (Wise et al., 1985). Because of this, they can reinforce an unequal power relationship between administrators and teachers (Starratt, 1992). Conversely, supervision looks to provide teachers with support and assistance in continually improving their practice (Berube \& Dexter, 2006; Donaldson, 2013; Marshall, 2012; McBride \& Skau, 1995; Starratt, 1992). Supervision is a formative process that focuses on professional development, identifying specific needs of individual teachers as well as those of the school as a whole (Borko, Elliot, \& Uchiyama, 2002; Hazi \& Rucinski, 2009). If the goal is to impact teaching behaviors in the classroom, administrators need to focus less on evaluation and more on providing ongoing supervision (Berube \& Dexter, 2006).

Creating a plan to help teachers strengthen their teaching practices necessitates an understanding of research regarding skill development in adults, and specifically teacher growth and development. Through their teacher training programs, pre-service teachers gain content knowledge, what to teach, and pedagogical knowledge, how to teach. When they start teaching, they must learn how to combine their pre-service training in content knowledge and pedagogical knowledge reflecting ways in which students learn and can be successful in the classroom (Segall, 2004; Shulman, 1986; Shulman \& Sherin, 2004). Several areas of theory and research speak to how teachers develop as practitioners. 
Dreyfus and Dreyfus (2004) proposed a five-stage model of adult skill acquisition ranging from novice to expert. However developmental stages should not be viewed as static, but as a developmental journey that teachers are continually undertaking (Berliner, 2001; Gossman, 2008). Part of this developmental journey includes the teachers' ability to reflect on what happens in the classroom and make appropriate adjustments to his/her teaching. Dewey (1933) discussed reflection as a process of developing habits of mind the ability to reconsider thoughts, concepts, and actions. Reflective habits of mind support teachers in moving beyond the technical knowledge and skills of teaching - the knowing-in-action (Schon, 1987) or narrative-in-action (Connelly \& Clandinin, 1985) to critically examine their pedagogy and think flexibly about unanticipated problems (Jones \& Vesilind, 1996). As teachers develop along the continuum from novice to expert, and reflect on their practice, they will begin to better assimilate past experiences to use when making decisions in their classrooms.

Schön (1987) has written extensively about the importance of reflection and becoming a reflective practitioner. He focuses on the importance of reflecting on mistakes or imperfections in a situation and learning from those mistakes. $\mathrm{He}$ differentiates between two different types of reflection. Reflection-on-action is reflecting on experiences that have taken place in the past whereas reflection-in-action is reflecting on an experience as it happens. As teachers progress through the developmental stages, they need to learn how to move from reflection-on-action to reflection-in-action. That reflection may lead to changing of schema and influence ways in which a teacher approaches similar situations in the future (Dewey, 1916; Schon, 1987). It is an important 
goal for all teachers to become reflective practitioners and reflect in practice (Geerinck, Masschelein, \& Simons, 2010; Schon, 1987).

When thinking about developing expertise in teaching, social interaction and exploring shared experiences with more capable peers is also key to adult learning and development. Reflection and interaction with others is essential for all learning because learning happens in a social context when individuals are communicating with each other (Dewey, 1916). As expert teachers share experiences, novice teachers are learning from and incorporating those shared experiences. With guided reflection, expert teachers can help make explicit the decisions they make throughout their lessons, which will, in turn, help novice teachers grow (Allen \& Casbergue, 1997; Cleary \& Groer, 1994; Jaeger, 2013).

All teachers need supports as they progress through the stages from novice to expert. To maximize support for all teachers, it is essential that administrators understand the types of support teachers need at various developmental stages (Peno \& Silva Mangiante, 2012). As teachers continue to progress through the stages of adult development their supports continue to change. They often no longer need formal external structures, but all "teachers need the support of at least some nearby co-workers who are trying to do the same things, and with whom they can share notes" (Duckworth, 2006, p. 9). Therefore, to enhance development, teachers need to be provided the opportunity and environment to reflect and communicate with each other. In order to achieve a shared sense of responsibility, the administrator must create a school climate in which collaboration and growth are supported. It must be one in which teachers feel 
comfortable taking risks and challenging themselves to learn without the fear of repercussions (Colton \& Sparkes-Langer, 1993; Duckworth, 2006).

Many administrators are tasked with the challenges of identifying and supporting teachers at various stages of development, while also evaluating and rating teachers using an evaluation tool. Although there is literature that looks at best practices in development of evaluation systems, as well as literature that investigates ways in which teachers grow and develop in their practice, there remains a disconnect between the ways in which evaluation practices and supporting teacher development play out in real world schools. Given the increased push for accountability and the high-stakes nature of recent evaluation systems, this disconnect is becoming more pronounced. Recent studies (Donaldson, 2013; Hazi \& Rucinski, 2009; Murphy et al., 2013; Starratt, 1992) have identified a conflict in the role of the administrator as the individual who is responsible for both evaluating and supporting teachers. Yet no research exists discussing how administrators manage these dual roles, and fulfill obligations both of evaluation and supervision. This research study intends to fill the gap in research by investigating the experiences of current Rhode Island administrators.

\section{Research Questions}

The goal of this research is to better understand how Rhode Island administrators balance the dual roles of evaluating teachers with the current RIDE high-stakes evaluation tool, and how the use of the evaluation system has influenced their role in supporting teachers in their building while creating a supportive environment. Specifically, this study will address the following research questions: 
RQ1: How do RI administrators who are responsible for both supervising faculty professional growth and conducting educator evaluations support the growth of their teachers?

RQ 2: How do administrators set the stage within the context of their schools to be able to supervise teacher growth and evaluate their teachers?

RQ 3: How does the RI educator evaluation system assist administrators in supporting faculty professional growth?

RQ 4: How does the RI educator evaluation system hinder or challenge administrators in supporting faculty professional growth?

\section{Definition of Key Terms}

To aid the reader, key terms used in the study are defined as follows:

Evaluation: a formal process developed by states or districts, and implemented by districts and schools, by which an evaluator, often a principal or other administrator, assigns a teacher a rating of effectiveness based on agreed upon measures.

Supervision: the process of supporting teachers' growth and development to improve teaching, with the end goal of positively affecting student achievement.

Induction coaching: releasing a teacher from all or part of his/her job to spend large quantities of time with a new teacher providing intensive support. Intensive training on coaching is often provided for the induction coach.

Mentoring: assigning an experienced teacher in the school or building to supporting a new teacher typically during after school hours and/or prep times. 
Student Learning Objectives (SLOs): element in the RIDE evaluation system. It is a long-term, measureable, academic goals set by teachers for students they instruct. They are intended to measure a teacher's impact on student achievement.

Professional Growth Goal (PGG): element in the RIDE evaluation system. It is a goal written by teachers to identify a measureable area of focus for professional growth over the course of the year of the evaluation cycle.

Educator Performance Support System (EPSS): online data support system for the RIDE educator evaluation system. EPSS is a way for teachers and administrators to share, collect and manage data related to the evaluation system.

Performance Improvement Plan: element of the RIDE evaluation system used specifically for teachers identified as Developing or Ineffective on a previous year's evaluation. It includes clear action steps and timelines for achieving those steps.

Final Effectiveness Ratings: according to the RIDE evaluation system four final effectiveness ratings are given which are, from highest to lowest, Highly Effective, Effective, Developing, and Ineffective.

Highly Effective: the highest rating that can be given to a teacher using the RIDE evaluation system, allowing teachers to be evaluated once every three years.

Effective: the second highest rating that can be given to a teacher using the RIDE evaluation system, allowing teachers to be evaluated once every two years.

Developing: the second lowest rating that can be given to a teacher using the RIDE evaluation system, requiring teachers to be evaluated every year and requiring teachers to be placed on a Performance Improvement Plan. 
Ineffective: the lowest rating that can be given to a teacher using the RIDE

evaluation system, requiring teachers to be evaluated every year and requiring teachers to be placed on a Performance Improvement Plan.

\section{Organization of the Dissertation}

This dissertation is organized into five chapters. Chapter 1 introduces the statement of the problem, purpose and significance of the study, research questions and definition of key terms. Chapter 2 is a review of the literature related to the theoretical framework for the study, as well as a review of literature related to effective teaching, teacher evaluation, teacher growth, supports necessary to facilitate ongoing teacher development, supervision, and the conflicting role of the administrator responsible for both evaluation and supervision of teachers. Chapter 3 provides a detailed description of the methodology used throughout the study as well as an introduction to the interviewees. Chapter 4 presents detailed findings from the interviews conducted and the artifacts collected. Chapter 5 presents a detailed discussion of the findings, limitations of this study, and implications and recommendations for future research. 


\section{Chapter 2: Theoretical Frame \& Literature Review}

\section{Background}

Education reform efforts have long identified student achievement as of vital importance, and have approached student achievement through a variety of different lenses. Some reform efforts such as No Child Left Behind (U.S. Department of Education, 2001) and Race to the Top (RTTT) (U.S. Department of Education, 2013) have focused on student achievement through the lens of curricular reform. Educational reform efforts have also capitalized on research related to the impact teachers make in the achievement of their students.

Wright, et al. (1997) found that the most important in-school factor effecting student achievement was the student's classroom teacher. They also cautioned about the negative effect that having an ineffective teacher has on student's academic progress. The authors suggested that a major component of teacher evaluations should include measures of student growth over time (Wright, et al., 1997). Ding and Sherman (2006) dove further into the topic of teacher impact on student achievement by examining the "relationship between teacher effectiveness and student achievement as measured by standardized test scores" (p. 40). In reviewing the literature related to the impact of effective teaching on student achievement, the authors identified a multilevel model. They acknowledge that an effective teacher impacts student achievement, but also noted that it is essential to take into account the variety of other factors that influence student achievement including student motivation, parent influence and involvement, as well as factors related to the school, the district, and the leadership. 


\section{Effective Teachers}

Brophy (1986) in summarizing research connecting teaching behaviors with student achievement also identified teacher effect as being measured by student achievement on standardized test scores, but differentiated that teacher effectiveness included developing students' personal and emotional skills in addition to their academic growth. Brophy identified teaching behaviors that were common for effective teaching, including quantity and pacing of instruction. He also described effective teaching practices as providing all students with the opportunity to learn, having clearly established and defined roles in the classrooms, setting high expectations, and focusing the majority of in class time on academic activities. To capitalize on instruction time, Brophy emphasized the relationship of student engagement and classroom management, as when teachers have highly effective classroom management practices, minimal instructional time is lost. Brophy identified other characteristics of effective teachers, such as that they provide positive supports for student growth, are well prepared, are continuously monitoring students, and have developed a skill for appropriate pacing of a lesson. They continue to set high expectations and provide students with an acceptable level of challenge. Whether in whole class, small group, or individualized instruction, effective teachers are actively engaged with their students and are personally ensuring that students understand the instructional content. When providing information, effective teachers are clear, enthusiastic, well structured, sufficiently redundant, and well sequenced. They are also skilled at questioning their students and ask questions at a variety of difficulty levels. They offer frequent feedback on answers, rather than just 
praise for correct answers. They also allow for sufficient wait time and call on a variety of students (Brophy, 1986).

Brophy attempted to differentiate between effective teaching behaviors and personality traits. He recognized that the teacher's personality and their development of the emotional climate of the room are also important for effective teaching practices. In addition to the importance of the teacher in regards to student achievement, he identified other factors that influence student achievement such as grade level, socioeconomic status, and student's ability (Brophy, 1986).

Evans (2002) identifying effective teachers as those that have an impact on student's standardized test scores, concurred that effective teachers are often found to be skilled at managing instructional time effectively, establishing and maintaining clear classroom routines, being well organized, having well defined classroom management practices, and providing continuous feedback to students. The traits identified as effective teaching behaviors have been used in teacher preparation programs, staff development programs, and as guidance for teacher evaluation programs (Evans, 2002).

\section{Measuring Teacher Effectiveness}

Even though it is challenging to identify, quantify, and ultimately measure a comprehensive list of effective teaching practices, it is still important to recognize the effect teachers do have on their students' academic and emotional development. The focus on ensuring effective teaching throughout our schools is not new. Since teachers are one of the biggest in-school factors that impact student achievement, many reform efforts have included some type of teacher evaluation systems as a way to identify effective teachers and ensure effective teachers in all of our classrooms. 
In 1983, a Nation at Risk (National Commission on Excellence in Education) recognized the importance of good teachers for student achievement and attempted to use teacher evaluation as a way to recognize and reward effective teachers. In addition to suggesting that overall teaching salaries be improved, it suggested that individual promotion, retention, and dismissal should be tied to an effective teacher evaluation system. No Child Left Behind (U.S. Department of Education, 2001) also emphasized the importance of having highly qualified teachers in every classroom and required schools to communicate to families the quality status of their children's teachers. One of the more recent federal reform efforts, Race to the Top (RTTT) emphasized that quality teaching makes a difference in the academic success of students, and required all states vying for RTTT grant money to develop a teacher evaluation tool as part of the grant process. RTTT suggested revising teacher evaluation practices to include compensation and retention policies as a way to reward effective teaching (U.S. Department of Education, 2013).

While evaluation systems do provide some measures for teacher quality, and there is research that suggests best practices for teacher evaluation, there is little evidence that ties evaluation to changes in teaching practice (Marshall, 2012; Murphy, et al., 2013; Starratt, 1992).

\section{Theoretical Framework}

When developing this study, there were several areas of research that garnered my attention. When looking at ways in which administrators support their teachers, it is important to have an understanding or framework for how teachers grow and develop. If administrators do not understand the ways in which their teachers develop, they will not 
know what types of supports they require. Research that addresses teacher evaluation tools and the impact they have on change in the classroom often addresses the climate and culture of a school. Without a positive school climate administrators are faced with the difficult challenge of impacting change, supporting teachers and evaluating teachers as well. Therefore, the two main theoretical frameworks I employed while designing this study focus on 1) adult learning theory and 2) social learning theory.

\section{Adult Learning Theory}

When teachers begin teaching, they have often developed some skills through their teacher-training program related to both content knowledge and pedagogical knowledge, what to teach and how to teach. Yet all teachers will need to continue to grow and develop in their practice. Therefore, administrators are often charged with the task of helping teachers develop their teaching practice. Adult learning theory helps us understand how adults develop as learners.

Dreyfus and Dreyfus (Dreyfus, 2004) suggested a five-stage model of adult skill acquisition, the stages being novice, advanced beginner, competent, proficient and expert. In each stage, adults show different ways of knowing. Applied to teacher development, the Dreyfus and Dreyfus (2004) model suggests that teachers in the novice stage will tend to follow rules they have learned in their teacher training and must learn to apply learned information to authentic classroom situations. They see things as black and white as they attempt to apply the knowledge they have gained from their teacher training programs (Dreyfus, 2004). Novice teachers focus on following rules and need to understand the application of new skills within the context of their own classroom in order to improve (Peno \& Silva Mangiante, 2012). 
As novice teachers progress to the advanced beginner stage they begin to differentiate situations and are better able to identify important versus unimportant information. When teachers gain this situational experience, they are better able to adapt and adjust instruction based on experiences with similar situations (Berliner, 2001; Daley, 1999; Dreyfus, 2004), but often struggle seeing the connection between distinct situations and do not always connect them as being similar (Berliner, 2001; Peno \& Silva Mangiante, 2012). Teachers at the advanced beginner stage are just starting to understand the ideas of self-monitoring and self-regulation (Daley, 1999).

"Although inexperience is equated perfectly with novice status in a field, the acquisition of experience does not automatically denote expertise" (Berliner, 2001, p. 464). It usually takes approximately three to five years for professionals to move into the competent stage. Competent professionals are better able to cope with unpredictable situations and even begin to plan for common misunderstandings and mishaps in the classroom. They are less reliant on external scaffolding of situations and are beginning to move toward self-regulation and self-monitoring (Berliner, 2001). Competent professionals are better able to identify, during instruction, those situations that are important and those that can be ignored (Daley, 1999). They are better able to assess the subtle ways in which situations are different and begin to take responsibility for both their successful and unsuccessful choices in the classroom. It is important at this stage for professionals to become emotionally connected and involved in their classroom decisions (Dreyfus, 2004).

This emotional attachment and reflection on decisions helps a teacher move into the proficient stage (Daley, 1999). As teachers become proficient, they are better able to 
assimilate their experiences and view their work in a more holistic way. Proficient teachers show control over their environment and are able to self-control and self-monitor their progress throughout a lesson. They are able to view similarities in situations that might previously have been viewed as distinct and different (Berliner, 2001).

The shift from proficient to expert teacher comes when a teacher not only sees the key elements of a situation, but also recognizes how to deal with those situations effectively. An expert teacher now has an intuitive grasp of what needs to be done and "does what normally works and, of course, it normally works" (Dreyfus, 2004, p. 180). Expert teachers have created a conceptual map of their classroom for themselves and are able to make interactive decisions throughout a lesson. They are able to focus on issues that directly relate to the lesson and not on those that will not impact instructional delivery (Cleary \& Groer, 1994).

The movement from novice to expert teacher requires several changes in teachers' performance. First teachers need to begin to move from abstract theory to concrete experiences. They need to learn to rely less on the training received and more on their own experiences, as well as the experiences of others that have been shared (Daley, 1999). Second, they need to move from seeing each situation as a separate experience toward seeing all situations as part of a larger whole. Finally, as teachers move from novice to expert, they need to shift from being an observer of what is happening in their classroom to an active participant. A major shift in psyche from a novice teacher to an expert teacher is the shift from fear of mistakes to the idea that mistakes are learning opportunities that help to expand their own repertoire of experiences (Berliner, 2001; Cleary \& Groer, 1994). 
While most beginning teachers often start their career as novices, not all veteran teachers are experts. Development along the continuum of novice to expert should not be viewed as linear but rather as a continuum that can and will be revisited throughout their career (Berliner, 2001; Gossman, 2008). "The key to expertise does not seem to reside in merely gaining experience, but in how the individual uses experience as a learning mechanism" (Ferry \& Ross-Gordon, 1998, p. 107). Expertise can also be domain or content specific.

Skill development, such as reflected in the Dreyfus, model is not the only type of adult development. There have also been a variety of researchers who have studied epistemological theories about knowledge and knowing. Perry, studying college-aged men, outlined a four stage developmental model of knowing (Tedesco, 1991). In the first stage, dualism, individuals are concerned with opposites such as right vs. wrong, good vs. bad, correct vs. incorrect. They see truth as inarguable and coming from authorities. Therefore, they see learning as a passive process. The second, multiplicity, is where individuals begin to see truths not as absolutes. They become functional skeptics and doubt authorities. Individuals in the third stage, relativism, begin to see truths through context. They see the importance of evidence or supporting material to defend a claim or belief. In Perry's fourth stage, commitment with relativism, individuals are willing to take a stance. Even though they understand there are no absolute right or wrong answers, they are able to use evidence and experience to make decisions and support their claims.

Belenky (Tedesco, 1991) identified stages of development known as Women's Ways of Knowing. Stage one, known as silence, is one in which women have no voice. Stage two is called received knowing, in which authorities are a source of truth and 
knowledge and there is only one right answer. Stage three is called subjective knowledge, in which truth is personal and private. Stage four, known as procedural knowing, is one in which individuals are looking for evidence to support their opinions and interpretations. Finally, stage five, called constructed knowledge, is the stage at which women are more intuitively constructing knowledge by integrating her thoughts and feelings (Tedesco, 1991).

King and Kitchener developed the Reflective Judgment model, which describes the developmental reasoning from adolescence to adulthood. The authors identified seven stages, clustered into three larger categories. The first category is pre-reflective thinking, where knowledge is absolute. Knowledge is given by authority and does not require justification or justification is provided through authority. The next category is quasireflective thinking in which individuals begin to question authority and knowledge becomes more situational. Knowing is justified through situational experience or context. The final category is reflective judgment in which knowledge is constructed through the evaluation of evidence and experience. Beliefs are justified based on the given evidence and experiences of the individual (King \& Kitchener, 2004).

Perry, Belenky, and King and Kitchener's (2004) models can be similarly compared. While Belenky's progression includes a pre-stage where women have no voice, the progression through the other stages is similar and moves from an initial stage where knowledge comes from authority, to a final stage in which decision making is based on situational evidence and an individual's own personal experiences.

These stages can also be compared to Dreyfus and Dreyfus' stages of skill development. At the novice stage, as well as the pre-reflective stage, individuals rely on 
set rules provided by authority. As individuals move through various stages of development toward quasi-reflective thinking, they begin to question authority and rely on personal experiences and context to make decisions and value judgments about a situation. Expert, reflective professionals use what they know to intuitively make and support their decisions (Dreyfus, 2004; Hofer \& Pintrich, 1997; King \& Kitchener, 2004). Table 2.1 shows the developmental stages of adult development identified by Dreyfus and Dreyfus alongside the epistemological stages identified by King and Kitchener, Perry, and Belenky as a means of comparison across the stages. 
Part of the developmental journey teachers must undertake entails their ability to reflect on what happens in the classroom and make appropriate adjustments. Dewey (1933) emphasized that reflection is a way of developing a practice of thinking and reconsidering all aspects of what happens within a teacher's classroom. As teachers develop reflective habits, these habits assist them in moving beyond the level of technical knowledge, to the application of that knowledge during in-class situations, to eventually being able to apply that knowledge to any type of situation (Clandinin \& Connely, 2000; Schon, 1987).

Schön (1987) has written extensively about the importance of reflection and becoming a reflective practitioner. He focused on the importance of reflecting on mistakes or imperfections in a situations and learning from those mistakes. He describes two different types of reflection. Reflection-on-action is reflecting on experiences that have taken place in the past whereas reflection-in-action is reflecting on experiences as they happen. Beginning teachers first need to develop skills related to reflecting-onaction in which they reflect on their lessons once completed. As they become more skilled at reflection, they will learn to reflect-in-action that is, reflect in the moment about potential strategies that might work to address situations as they arise (Schon, 1987).

It is an important goal for all teachers to become reflective practitioners and reflect in practice (Geerinck, et al., 2010; Schön, 1987). Experienced teachers are more aware of what is happening in their classroom and are better able to reflect on, and make adjustments to, their own teaching in the moment (Cleary \& Groer, 1994). Novice teachers often struggle with reflecting on situations accurately. They need guidance deciding between the important and unimportant details in their classes, as well as how to 
make in-class adjustments to teaching (Berliner, 2001; Dreyfus, 2004). While selfreflection is an important part of developing as an educator, it is also important that teachers have the opportunity to reflect with others (Allen \& Casbergue, 1997; Cleary \& Groer, 1994; Colton \& Sparkes-Langer, 1993).

A teacher progresses through the various developmental stages of learning and it is important for the administrator to know and understand where each teacher is developmentally in order to provide support at the level appropriate for the individual teacher (Peno \& Silva Mangiante, 2012). It is also important to note that teachers are in various stages on the novice to expert skill model (Dreyfus, 2004) throughout their teaching career, but teacher evaluation models tend to focus on expert teaching skills leaving little room for identifying and supporting growth for the novice or advanced beginner. In addition, receiving a rating of less than effective is seen as negative by many teachers (Starratt, 1992).

\section{Social Learning Theory}

While like adult learning theory provides a framework for studying how teachers learn, social learning theory provides us with a basis for how adults learn within a social environment. Social interaction and exploring shared experiences with more capable peers are keys to learning and development. Reflection and interaction with others is essential for all learning because learning happens in a social context when individuals are communicating with each other (Dewey, 1916). Dewey identifies the idea that learning is social and emphasizes the notion that communication is a vital part of learning for all members of society. "Communication is a process of sharing experiences till it becomes a common possession" (p. 9). Dewey recognizes that more mature members of a 
community, such as expert teachers in a school, must assist in the development of immature, or novice members. As expert teachers share experiences, novice teachers are learning from and incorporating these shared experiences into their experiences. Expert teachers can help make explicit the decisions they make throughout the lesson, which will, in turn, help novice teachers grow (Allen \& Casbergue, 1997; Cleary \& Groer, 1994; Jaeger, 2013).

Vygotsky (1978) also emphasizes the social construction of knowledge and how it helps us understand how learning occurs. He states that learning is a social activity and is most effective when it happens through everyday experiences. He talks about the difference between actual developmental level and potential developmental level. Actual developmental level is the independent level at which an individual can accomplish a task. The potential developmental level is that which can be accomplished through collaboration with more capable peers. Vygotsky (1978) identifies the distance between that actual developmental level and the potential developmental level as an individual's Zone of Proximal Development (ZPD). Teachers learn when working with colleagues who are more advanced than their own ZPD. Thus, Vygotsky's work emphasizes the importance of individuals developing new skills by interacting and collaborating with more capable peers. The more capable peers provide the scaffolding necessary for less experienced learners to achieve success at new tasks and in turn helps them to progress developmentally (Kim \& Baylor, 2006; Peno \& Silva Mangiante, 2012; Vygotsky, 1978).

As more experienced or expert teachers share experiences, less experienced or novice teachers are also learning from the shared experience. This shared experience helps all teachers grow (Allen \& Casbergue, 1997; Cleary \& Groer, 1994; Colton \& 
Sparkes-Langer, 1993; Munby, Russell, \& Martin, 1997). There are a variety of models of ways of developing social learning opportunities for teachers, fostering a collaborative culture, and developing a feeling of collective responsibility.

Lave and Wenger have used the term Community of Practice (CoP) to describe a group of professionals working together around a topic of common interest. They brought the idea of $\mathrm{CoP}$ to the field of teaching and learning stating that they are naturally occurring throughout society because we, as humans, are constantly developing relationships with others to address and solve problems of interest to us. They believe that teaching and learning are social events that occur formally and informally in society. Using Lave and Wenger's idea of a CoP, a specific community of educators working together on their practice of teaching would focus not only on the social process of learning but on the learning and sharing of new skills (Printy, 2008; Smith, 2003).

CoPs can be naturally occurring or formally created, but is defined along three dimensions: 1) what it is about 2) how it functions and 3) what capability it has produced. CoPs are developed around ideas that matter to a group of people. People are brought together to address shared problems or concerns and provide support and mutual sharing of knowledge and skills (Smith, 2003). Leadership within a CoP is often distributive and naturally occurring. It is emergent and fluid with different members taking on the leadership role at different times based on the problem to be solved. Being active participants in a CoP implies a level of mutuality in which all individuals within the community of practice benefit from the shared resources as well as make active contributions to it (Printy, 2008). Administrators take an active role in the CoP through the way in which they shape and lead conversations, the type of environment that is 
created in the school within which the CoP functions, and the type of support and direction provided for the CoPs. In a properly functioning CoP, administrators take a backseat to the naturally occurring leadership within the CoP (Printy, 2008).

\section{Implications of Theory and Research on Supporting Teacher Growth}

Administrators are often responsible for both supporting and evaluating their teachers. This dual role requires that administrators have an in-depth knowledge of teacher development and understanding of the types of support teachers require at various developmental stages.

The Dreyfus and Dreyfus model of skill development has been applied to classroom practices in a variety of ways. Studies have looked at the various ways novice and expert teachers differ (Berliner, 2001). When making decisions about the importance of what happens in the classroom related to students, content, and procedures, expert teachers have been found to make many more interactive or "inflight" decisions about what happens in their classroom than novice teachers (Cleary \& Groer, 1994). This interactive decision-making is something that needs to be directly modeled, shared, and taught to novice teachers.

Teachers who are at the beginning stages of development often need external support such as mentoring or induction coaching (Moir, 2010; Peno \& Silva Mangiante, 2012). The major difference between induction coaching and mentoring is that often times induction coaches are released from all or part of their job to spend large quantities of time with their new teachers, while mentors are usually other teachers in the school or district and work with the new teacher during after school hours and/or prep times. Programs such as mentoring and induction provide support for new teachers by allowing 
them the opportunity to work with and learn from experienced teachers (BambrickSantoyo, 2013; Moir, 2010; Rhode Island Department of Education, 2011).

The success of these programs varies depending on the structure and design of the mentoring or induction program itself. This support can take place in a variety of ways but might include observations -both the new teacher observing the experienced teacher and vise versa, co-planning, co-teaching, reflecting together, assessing and analyzing student work, sharing documents for students and/or parents, and helping the new teacher set up his or her classroom. Many schools have mentoring or coaching programs in place for new or novice teachers and they have been shown to be beneficial in supporting and retaining new teachers (Bambrick-Santoyo, 2013; Moir, 2010).

A novice teacher can get caught up in the novelty of a situation because they do not know how to apply the rules they learned in pre-service, while an expert teacher uses that novelty to reflect on what has worked for them in past situations and adds the new situation to his/her existing schema. When working with a novice teacher, the more experienced teacher, as mentor, can ask guiding questions about unfamiliar situations and help the novice teacher to further develop her/his own schema to address such situations (Peno \& Silva Mangiante, 2012). The goals of mentoring novice teachers are to assist them in examining rules they have been taught in training programs, guide them through reflection, and provide specific and timely feedback (Peno \& Silva Mangiante, 2012). While novice teachers can often benefit from support such as mentoring or induction coaching, as teachers gain more experience the types of support they need differ. Teachers in the advanced beginner stage are in a transitional phase in which they require less external support, but scaffolding should still be available. Peno and Silva 
Mangiante (2012) offer a model for ongoing mentoring for teachers at all stages, the Purposeful On-going Mentoring Model (POMM). The model focuses on ways to assist teachers from moving from novice toward expert teacher. The model emphasizes the importance of looking at the developmental level of mentors and mentees as well as intentionally setting goals and providing scaffolding to assist movement along developmental levels.

When attempting to support teachers from novice to expert, mentors must be intentionally chose and provided with guidance on how to support their mentees. It is essential for mentors to understand the needs of their mentee and the types of supports they need in order to help them progress developmentally (Peno \& Silva Mangiante, 2012). When choosing mentees, it is important to keep in mind Vygotsky's emphasis on the importance of interacting with more capable peers within one's Zone of Proximal Development (ZPD). Administrators understanding the various developmental stages of their teachers will assist them in pairing teachers with peers who are at the appropriate ZPD to help them to develop their skills (Colton \& Sparkes-Langer, 1993; Vygotsky, 1978).

As teachers continue to progress through the stages of adult development, the supports they need continue to change as well. All "teachers need the support of at least some nearby co-workers who are trying to do the same things, and with whom they can share notes" (Duckworth, 2006, p. 9). Social interaction and exploring shared experiences with more capable peers is key to learning and development. Reflecting on these experiences with others is also an important part of teacher development (Vygotsky, 1978). Given the social nature of learning and the importance of experienced individuals 
reflecting with less experienced individuals, schools need to provide teachers with the opportunity and environment within which to reflect with their colleagues. An experienced teacher has a more vast and varied schema to reflect on and can make more informed decisions than a beginning teacher (Allen \& Casbergue, 1997; Cleary \& Groer, 1994; Jaeger, 2013) and therefore can assist these more novice teachers.

Reflection with others can also be used as a tool for an entire school community to move from focusing on individual teacher success in the classroom, to building a climate and culture of shared responsibility for the success of all students' achievement within the school (Borko, et al., 2002). In order to achieve a shared sense of responsibility, administrators must work towards a school climate in which collaboration and growth are supported. The school climate must be one in which teachers feel comfortable taking risks and challenging themselves to learn without the fear of repercussions (Colton \& Sparkes-Langer, 1993; Duckworth, 2006).

\section{Implications of Theory and Research on Effective Teacher Professional}

\section{Development}

Professional development (PD) models have been created to help teachers grow as professionals. For PD to have an impact on teaching practices, it is important to have teacher buy-in (Gurskey, 1986). When reviewing PD models, it is important to recognize the work of Knowles (1973) on Andragogy, the art and science of teaching adults. In his theory on Andragogy, Knowles identifies six key principles delineating how adults differ from K-12 learners. These principles include the role of experience, self-directedness, a need to know, readiness to learn, orientation to learning, and intrinsic motivation. Adults are motivated to learn based on problems or situations that arise in their own work or life 
experiences that cause them to want or need to learn something. Adults are more likely to want to learn something when they can take their own experiences and discuss them with others. They also want to be able to choose what they are going to learn or study and want it to be applicable to what they are currently doing on their job or in their lives (Knowles, 1973).

Teachers are more committed to learning that which they consider valuable and important to them. Teachers need to be a part of the planning and development process of professional development programs and activities. When creating and implementing professional development that focuses directly on teaching practices, it is important to understand that change of practice takes time and teachers should be afforded that time to work on improving their practice (Knowles, 1973; Wise, et al., 1985). Programs should be clear and have personal connections to what the teachers are doing in practice. It is also important to provide teachers with on going feedback and support to ensure that new learning is being practiced and incorporated into regular teaching practices (Gurskey, 1986). Administrators need to understand the social nature of learning and allow teachers the opportunity to learn and share ideas with each other (Gurskey, 1986).

The type of activity that teachers engage in during professional development is important to its success (Garet, Porter, Desimone, Birman, \& Yoon, 2001). When activities are presented as one-shot workshops, they are less likely to impact teachers' practice (Donaldson, 2013). Therefore not only the style of the activity is important, but the duration of the activity is also key to lasting reform (Borko, et al., 2002). Professional development activities should promote active learning from teachers. Some ways in which to do that include observing and being observed by peers, providing time for 
planning and then reviewing classroom implementation of ideas with peers, or reviewing student work (Clarke \& Hollingsworth, 2002; Garet, et al. 2001). For administrators, it is important to get teachers involved in creating, leading, developing, and sharing their own professional development activities as a way to impact teacher growth in their classrooms. It is essential to focus on collective responsibility of the school and helping all teachers collaborate to impact teachers' behaviors in the classroom (Garet, et al., 2001).

Borko et al. (2002) emphasized, while professional development is key to helping teachers change, it often receives inadequate support in terms of time and resources, both financial and material. When looking at reform efforts, they recommended that schools focus on: 1) individual teacher knowledge, skills and disposition, 2) professional community and 3) program coherence. Teachers need to feel as if they have the opportunity to influence school practices and policies as part of the school professional community. Administration must support teacher growth through professional development activities deemed essential by the teachers and the professional community. But often reform acts are presented as pressure to change and teachers are held accountable through evaluation programs rather than supported via professional development (Borko, et al., 2002).

Clarke and Hollingsworth (2002) have also emphasized the importance of developing a supportive culture as a way of encouraging teacher change. The authors encourage the shift "from programs that change teachers to teachers as active learners shaping their professional growth through reflective participation in professional development programs and in practice" (p. 948). They also recognize the importance of 
both the sustained nature of professional development and the social aspect of helping teachers change. While it is easy to recognize that students learn differently, it is also important to recognize that teachers learn differently and are at different places developmentally therefore they require differentiated support and PD opportunities (Clarke \& Hollingsworth, 2002).

The concept of developing a community of professionals working together is essential to the growth of all teachers (Gurskey, 1986; Printy, 2008). When addressing ways to help teachers continue to improve and develop their practice, it is important to engage teachers in a community of learners. A community of learners needs to have teachers actively involved in addressing their own concerns in their classrooms.

Developing a professional community within the school in which there are shared goals, collaboration, and opportunity for reflection, will increase teachers investment in their own growth within their school. When staff is provided with both a centralized and decentralized approach to professional development, they are able to participate both personally and as part of a group and guide their own learning (Borko, et al., 2002).

Professional development opportunities for teachers should allow for ongoing collaboration with peers that focus on a sense of collective responsibility for the success of all students. Allowing teachers the opportunity to work with peers who have the same students, or teach the same content, or have the same professional growth goal, creates a more engaging environment in which teachers have investment in their own professional development (Garet, et al., 2001).

Social learning and opportunities to reflect with other teachers to work on addressing problems or concerns are vital for teacher growth (Gossman, 2008; Kim \& 
Baylor, 2006). Teachers need to identify the conflicts or challenges they are facing, discuss and reflect on those challenges, try something new, then come back and reflect and discuss again. By putting theories into practice, teachers are better able to see the results of what they are doing and will continue to grow in their own professional development (Clarke \& Hollingsworth, 2002).

\section{Administrators' Role in Professional Development and Evaluation}

Administrators serve many roles within their school communities but two of the main roles are evaluating their teachers and facilitating teachers' professional development. There is research (Borko, et al., 2002; Clarke \& Hollingsworth, 2002; Garet, et al., 2001) about the ways in which adults learn and how that applies to teachers' development within their profession as well as the role of the administrator in that development (Berube \& Dexter, 2006; Donaldson, 2013; McBride \& Skau, 1995). Research emphasizes the importance of creating an environment of trust and empowerment for teachers as a foundation for growth and both individual and collective development (Donaldson, 2013; McBride \& Skau, 1995; Starratt, 1992; Sullivan \& Glanz, 2000).

The role of the administrator as a supervisor is the role of helping a teacher to develop professionally, while the role of an evaluator serves more of a personnel function of rating teachers and making recommendations for retention and hiring (Hazi \& Rucinski, 2009). Measuring teachers and developing teachers serve two different purposes (Marzano, 2012). This conflict of evaluation and supervision is long-standing and must be navigated by administrators on a daily basis.

Berube and Dexter (2006) provide clear, distinct definitions of evaluation and 
supervision. Evaluation is seen as a formal process developed by states or districts, and implemented by districts and schools, by which an evaluator, often a principal or other administrator, assigns a teacher an end of the year rating. It can also be used to determine whether a teacher will return to that school the following year, whether a teacher will be put on an improvement plan, and the degree to which s/he will be evaluated the following year. The evaluation process is intended to ensure quality teaching. It is a summative system in which the various elements of the evaluation system combine to provide a final judgment or overall rating (Berube \& Dexter, 2006)

Supervision is the process of involving teachers in professional growth with the purpose of improving teaching and increasing student achievement. Quality supervision is an ongoing process that often includes school administrators, central office personnel, department chairs, teacher leaders, and, most importantly, teachers themselves. Supervision is designed to promote growth, development of all teachers, and a culture of problem-solving and collective responsibility. Supervision is formative, focuses on ongoing development, includes differentiated approaches for different teachers (Berube \& Dexter, 2006).

Even with identified best practices of teacher evaluation and extensive knowledge about adult development, we are still struggling with how to impact teacher growth and development in our schools. It is the job of the administrator to ensure that effective teaching is happening in their schools. But current evaluation systems are viewed as "flawed, contested and problematic" (Hazi \& Rucinski, 2009, p. 3). Through the evaluation process, administrators are only able to witness a tiny amount of instructional time in an isolated setting, which often leaves them with an incomplete picture of what is 
happening in the classroom. High stakes evaluations shut down adult learning and reinforce teacher isolation (Marshall, 2012).

Donaldson (2013) identifies limitations evaluations have on impacting teaching practice in the classroom, and factors that might contribute to their lack of impact, from evaluations, such as the evaluation tool itself, training provided for the tool, oversight for the evaluation process, a culture of "nice," limited impact on removing or remediating ineffective teachers, and extensive time needed to complete the evaluation tool effectively (Berliner, 2001).

High stakes evaluations are present in most educational systems today. But research has shown that teacher evaluation as currently constituted has little impact on school improvement (Murphy, et al., 2013). "Building principals are charged with helping teachers improve instruction and learning while holding teachers accountable for student achievement” (Ferry \& Ross-Gordon, 1998, p. 12). Administrators need to identify ways to support their teachers while also conducting high stakes evaluations.

\section{Deficiencies in the Literature}

Research suggests why we should evaluate, as well as provides guidance in what makes the best evaluation practices. Educational policies and practices have defined and refined what it means to be an effective teacher and have attempted to align those best practices with evaluating and identifying effective teaching. But there is a disconnect between evaluating teachers and helping them to become effective teachers. Even the best evaluation systems are flawed and problematic (Hazi \& Rucinski, 2009). Evaluation causes a negative power dynamic between teacher and administrator (Daley, 1999). Little 
evidence has been provided that links teacher evaluation to a change in teaching practices (Daley, 1999).

An additional complication is the fact that the individual evaluating the teacher, often the administrator is also the individual who is charged with creating a supportive environment that encourages the teacher to improve practice in her/his classroom. Recent studies have identified this conflict for administrators acting in the dual roles of both supporting and evaluating their teachers (Daley, 1999; Ferry \& Ross-Gordon, 1998; Hazi \& Rucinski, 2009; Sullivan \& Glanz, 2000). The school administrator plays a key role in both evaluating and supporting teachers, but given the increased push for accountability and the high stakes nature of recent evaluation systems, this conflict is becoming more pronounced. This study intends to look at how current Rhode Island administrators are balancing these dual roles. 


\section{Chapter 3: Methodology}

\section{Introduction}

The purpose of this qualitative, phenomenological study is to gain an in-depth understanding of how Rhode Island administrators construct their reality of implementing the Rhode Island Department of Education (RIDE) high stakes evaluation system, while also supporting teachers toward improving practice and professional growth. The research questions have a social constructivist worldview in which there are multiple meanings and constructions of an event. Social constructivism is focused on how people within a particular setting or environment construct or create their own reality, which is best examined through qualitative research practices. This type of research lends itself to open-ended interviews that allow participants to share and retell the stories about their experiences (Creswell, 2009; Merriam, 2009). The specific qualitative method this study uses is phenomenology. Phenomenological research focuses on the essence of a particular experience, or phenomenon, as described by the participants (Creswell, 2009; Fraenkel, et al., 1993). The phenomenon to be studied is implementing the Rhode Island educator evaluation system and supporting teacher growth as a Rhode Island administrator.

Phenomenology utilizes in-depth interviews with individuals who have experienced the phenomenon to best understand their experiences (Patton, 2002). The data from those interviews is then analyzed to identify any overall commonalities of the experience. The researcher uses the data to describe the ways in which most, if not all, participants experienced the specific phenomenon. Themes and essential ideas are reported out as a narrative description of the phenomenon (Fraenkel et al., 1993). 


\section{Research Questions}

This research study is designed to better understand how Rhode Island administrators balance the dual roles of evaluating and supporting their teachers with the current RIDE high stakes evaluation tool. It examines how the use of the evaluation system has influenced their role in assisting teacher growth as well as creating a positive and trusting environment. Specifically, this study will address the following research questions:

RQ1: How do RI administrators who are responsible for both supervising faculty professional growth and conducting educator evaluations support the growth of their teachers?

RQ 2: How do administrators set the stage within the context of their schools to be able to supervise teacher growth and evaluate their teachers?

RQ 3: How does the RI educator evaluation system assist administrators in supporting faculty professional growth?

RQ 4: How does the RI educator evaluation system hinder or challenge administrators in supporting faculty professional growth?

\section{Role of the Researcher}

As both the researcher and an insider to this research, it is important to clearly state my role. I am an administrator in Rhode Island who is tasked with the dual roles of evaluating my teachers using the RI educator evaluation system while also supporting my teachers' professional growth by developing, providing, and fostering professional development activities and creating a culture that supports growth. This is my seventh year in the role of administrator at my current school, and the fifth year that I have been 
using the RIDE-created evaluation tool to evaluate my teachers. In addition to being an administrator in RI, I am also the main researcher for this study and the primary individual conducting interviews and analyzing data for this study.

Researcher bias is a common concern when conducting research using phenomenological interviews. One way of dealing with researcher bias typically practiced in phenomenological studies is engaging in a process called epoche or bracketing (Moustakas, 1994). Epoche is a Greek word that means to refrain from judgment. In phenomenological research it is the specific act of the researcher becoming aware of his or her personal bias and putting aside, or bracketing, those feelings. It is a process the researcher engages in to remove, or at least identify and become aware of, her own prejudices, viewpoints or assumptions regarding the phenomenon under investigation (Moustakas, 1994; Patton, 2002).

Moustakas suggests a researcher should engage in epoche by going through the interview process that her research participants will go through before conducting the interviews (Moustakas, 1994). I asked a colleague to interview me using the proposed interview protocol. The process of completing the interview protocol helped me in several ways. First, it allowed me to discuss the various ideas and concepts that I am researching and document my own feelings and beliefs about the evaluation system and ways of supporting my teachers through a formalized process. Second, it allowed me the opportunity to review my questions and determine if they were prompting an interviewee to discuss the key concepts I was interested in learning about. After going through the interview myself as the interviewee, I realized that the ordering of questions was critical. Placing question about evaluation system first within the protocol led the interviewee to 
then discuss later questions about support through the lens of evaluation, rather than discussing support outside of the evaluation. I reordered the interview protocol and put the questions about support and professional development first, following them up with questions about evaluation, then with questions about conflicts or successes, and finally questions about ways interviewees have found to balance support and evaluation.

Another way I worked to identify my role as a researcher and make my beliefs transparent was in keeping a journal of field notes. This helped me to document in my own words my feelings and reactions to interviews, as well as events that happened during the interview process, transcription process, and throughout the research process (Merriam, 2009).

\section{Participants}

Participants for this study were selected through purposeful sampling, which is the process of selecting participants because of their experience with the topic and the information they can share (Patton, 2002). I was specifically looking for current RI administrators who are using the RIDE developed RI educator evaluation system, and who have been at their schools for at least two years. I chose two years because it is important for administrators to have had the opportunity to fully experience the evaluation cycle more than once in order to best identify how they can balance the ongoing benefits and challenges presented by the use of the educator evaluation system.

To obtain my sample, I first determined which of the fifty-six schools/districts in RI use the RI Teacher Evaluation System. Although RIDE created one system that most districts use, there are other RIDE-approved options that districts can use. I contacted RIDE to obtain a list of all districts/schools in Rhode Island, and which teacher 
evaluation system they are using. Based on the information provided, I eliminated nine schools/districts that are not using the RIDE model. I also eliminated my own district, due to potential issues with bias. That left forty-six remaining districts. From those I eliminated eighteen charter or specialty schools to focus specifically on public schools that have more similar profiles and guidelines, leaving twenty-eight districts. Finally, I eliminated five districts that had less than one hundred people total being evaluated in the district, because the number of people evaluated in the district will have a direct impact on how an administrator will balance evaluation and support of teachers including time devoted to evaluation and support.

This gave me a final list of twenty-three districts that I deemed eligible to participate in my research. I then went online and searched for the names, email addresses, and contact numbers of each school and administrator in each of the qualifying districts. There were a total of ninety-six schools at all levels. However, I was unable to get contact information on all of the administrators. As a first course of contact, I directly emailed all thirty-six administrators whose email addresses I was able to collect (See Appendix A).

In the initial email sent to administrators, I explained the study and included a link to a survey that those interested were asked to complete. The survey (See Appendix B) requested the administrators' contact information, school and district information, and consent to be contacted. It also posed questions to ensure that the administrator met the qualifications of (a) having been at their school for at least two years, (b) using the RI educator evaluation system, and (c) being responsible for both roles of evaluating and 
supporting teachers within their district. From the initial email, two administrators responded immediately. I contacted both administrators via email and phone.

Through interviews with those initial administrators who agreed to be participants, I then used snowball sampling to reach out to other administrators who might be willing to be interviewed. Snowball sampling is the process of asking individuals to provide names of other individuals they think might be knowledgeable about the topics being covered in the research study (Patton, 2002). The first administrator interviewed suggested two other administrators at two different schools who both qualified for the study. I contacted both administrators to see if they were interested in participating in the study.

The first administrator referred responded positively to my email request, and also recommended a colleague, an assistant principal for teaching and learning. I arranged an interview with both the principal and assistant principal, who requested that they be interviewed together since they work as an administrative team and wanted to have the opportunity to answer questions together. The second administrator recommended to me from the first participant also completed the survey and qualified for the study. I contacted the administrator and we set up an interview time.

The remaining administrators were identified through snowball sampling. When contacting each individual administrator, I explained the informed consent process and offered to send the informed consent form via mail (See Appendix C). All administrators elected to receive the consent form at the time of the interview and signed it before the interview began. Administrators were given a copy of the signed form and I retained a copy for my records. 
There is no specific guidance for sample size in qualitative analysis. Given the indepth nature of the interviews, a smaller sample size is more manageable and tends to provide in-depth information relevant to the phenomenon being studied (Patton, 2002). When determining the number of participants for the study I was looking for representation from administrators from a variety of grade levels. The six administrators I interviewed covered all grade levels from kindergarten through twelfth grade.

Six individuals participated in the research study, four principals and one principal and assistant principal pair. Table 3.1 summarizes data related to administrators interviewed.

Table 3.1

Summary of Interviewee Data

\begin{tabular}{llllll}
\hline Pseudonym & Gender & Grade level & $\begin{array}{l}\text { \# of } \\
\text { Teachers }\end{array}$ & $\begin{array}{l}\text { \# of } \\
\text { Students }\end{array}$ & $\begin{array}{l}\text { Administrative } \\
\text { Team }\end{array}$ \\
\hline Margaret & Female & High School & 120 & 1350 & $\begin{array}{l}\text { Principal \& } \\
\text { 2 Assistant Principals }\end{array}$ \\
\hline Becky & Female & High School & 115 & 1350 & $\begin{array}{l}\text { Principal \& } \\
\text { 2 Assistant Principals }\end{array}$ \\
\hline Thomas & Male & High School & 115 & 1350 & $\begin{array}{l}\text { Principal \& } \\
\text { 2 Assistant Principals }\end{array}$ \\
\hline Alicia & Female & Elementary & 25 & 275 & Principal only \\
\hline Garry & Male & Elementary & 20 & 200 & Principal only \\
\hline Zachary & Male & Middle & 110 & 950 & $\begin{array}{l}\text { Principal \& } \\
\text { 1 Assistant Principal }\end{array}$ \\
& & & & &
\end{tabular}

Three interviewees were female and three were male. All of the administrators interviewed are responsible for evaluating their teachers using the current RIDE educator evaluation tool. The number of staff evaluated by administrators varied from approximately twenty to approximately one hundred twenty. Two of the administrators with the smallest number of teachers, both under thirty teachers, are the sole administrators in their buildings. The other four administrators all have over a hundred 
teachers to evaluate and have an administrative team of one principal and either one or two assistant principals who help with evaluations. In addition to evaluating, all of the administrators are also responsible for supporting their teachers' growth, planning professional development activities, and acting as instructional leaders in their schools.

Five of the interviews took place at the administrators' schools at a time that was convenient to them. One interview was conducted at a local coffee shop at the suggestion of the administrator. Interviews were audiotaped on my iPad and later transcribed on my laptop. Most communication was done through email with emails being sent to confirm interview time, follow up on completed interviews, request or receipt of professional development documents, and sharing and discussing transcription, analysis and findings.

Confidentiality of participants and their schools was maintained throughout the research study. Pseudonyms were used for all participants. General information about all of the schools, grade levels and districts, was complied. There was representation from two high schools, one middle school, and two elementary schools. All of the schools were located in suburban districts.

Below is a brief introduction to each of the administrators interviewed (all names are pseudonyms).

\section{Interviewee \#1: Margaret}

Margaret has been the principal of a suburban high school for four years. She has approximately 1350 students at her school and approximately one hundred twenty faculty members, inclusive of teacher assistants, secretaries, and other support staff. She recognizes that the size of the school allows for a variety of clubs, sports and activities for students. "Everyone has a group or people that they can enjoy them so nobody is by 
themselves." She and her faculty always work on personalization for the students to best meet their needs.

Margaret's administrative team is made up of one principal, herself, and two assistant principals. She and her administrative team split up evaluations amongst the three of them. They meet to communicate expectations and ensure that they are consistent in conducting and completing evaluations.

Margaret identifies the administrative team in her school as a positive for her teachers. She emphasizes the importance of having the same administrative team together and having an opportunity to meet and plan. "We met last week for three days." Whenever the administrative team meets and plans, "it always, always revolves around the students." Having a consistent administrative team is important to help plan and organize the activities and supports they provide for teachers. "This is the first year, I'm going into my $4^{\text {th }}$ year, that I think I'm finally kind of planned out."

Margaret has shifted her staff meetings away from the more traditional, informational faculty meeting and instead uses technology to share information with staff. "We haven't done informational faculty meetings for a few years. I do a blog on Fridays and I send all my information out on the blog." Her meetings are centered on professional development focus areas the administrative team has determined for the year, shifting the meeting format. The meetings are run either by the administrative team or the instructional leadership team. "We're going to always be there to help them, but it's not going to be us standing up front presenting."

Margaret identifies herself as a "yes first" leader. "The thing about my leadership style is that I'm basically 'Yes, let's see how it fits. Yes, let's see the policy. Yes, let's 
see this." She starts with a yes "if it makes sense" and then checks with the policy to ensure that what is proposed can actually happen. She feels that "always saying no first" would limit the things that happen in her school and the risks that her teachers would be willing to take.

While Margaret identifies as a yes-first leader, she also states that she holds her teachers accountable. She does not allow her teachers to "walk all over" her. She stresses that, "there is nobody here in this building that thinks they can skate." She feels that her teachers know that she is, "here to totally support them $100 \%$." She often asks teachers, "What do you need? How can I help? Do you want to be on this committee? Would you like to do this?" But she recognizes that if teachers are going to wait to be paid for every extra opportunity that arises, than "a lot of opportunities are going to pass them by." She sees herself as a change agent and emphasizes that she is, "not going to stay business as usual."

Margaret also recognizes the importance of displaying a positive attitude to her teachers. Part of her leadership style is that she makes a conscious decision to be happy at work. 'I am a happy person and one of my blogs was, 'I choose happy.' I do. I choose to be happy and it's work." In addition to choosing to be happy, she also chooses to not focus on the negative aspects of her job. "I don't sit there in the hallway and talk to people about what my mandates are." She also tries "to value people's time and I feel like they should too."

Margaret is very passionate about her school. She repeatedly states, "I love my school." She loves the positive attitudes of the faculty and students at her school. She identifies a positive attitude among "the teachers and the administration and the students 
and the staff, everybody, just, it doesn't matter who walks through the door, that is why I think I love this school so much." If she could describe her school's mission statement in just "a couple words, I really think our mission statement around here should be student success." She feels that is what she and her faculty focus on at all times.

Margaret also has a positive attitude about her school and her job, "I happen to like what I do. I really love it. I have my times, I'm just not going to sit there and bitch all day." As an administrator she recognizes the importance of being visible in her school. She states that early in the year it is easy to get into everyone's classroom for nonevaluative purposes, but as the year goes on she tends to get busy. She says, "one of my goals last year was $50 \%$ of the time to be either in people's classroom or side by side, shoulder to shoulder, helping them with their professional development." She admits that she did not meet that goal, but continues to try to be present and spend her time with teachers when she is in the building.

Margaret identifies her central office administration as supportive. She states, "My assistant superintendent and superintendent are so supportive, and the business manager who holds all the money, so, so supportive when we do these things." She also identifies that when she brings an idea to her superintendent or assistant superintendent, "and I bring evidence and specificity, they are $100 \%$ 'go for it,' but it has to get through the school committee body. School committee can be tough, but also when they support you they are supportive."

\section{Interviewees \# 2 and \#3: Becky and Thomas}

Becky is the assistant principal and Thomas is the principal of a suburban high school with approximately 1350 students, one hundred fifteen teachers, and 
approximately two hundred faculty, inclusive of TAs and other support staff, at their school. They requested to be interviewed together after they discussed with each other the fact that they had both completed my interview survey. They worked together to coordinate the interview time. Due to scheduling constraints I began the interview with Becky and Thomas joined us twenty minutes into the hour and fifteen minute long interview. They recommended including their other assistant principal as well, but he was away on vacation at the time of the interview.

Becky recognizes the importance of making sure all students are connected to adults within the school. "We do a students connections survey to see who students are connected to adult-wise and student-wise in the building. Another district kind of rolled it out and we kind of dovetailed it and it's helped us a little bit, actually it's helped us a lot in making sure that kids are connected. They have also been able to move past that and find out, at the beginning of the year, who's not connected and connecting them with support staff to then support them."

There is a third assistant principal who also works with them as part of the administrative team. They split up evaluations between the three administrators and they also have "one special education department chair that [evaluated] a lot of the special education teachers this year. She does the whole thing and we sort of finalize it."

Thomas always tries to be supportive of his teachers by being a "yes first" leader. When somebody "comes in here and says to me, 'I want to do a color run fund raiser.' 'OK.' Or 'We want to try this with the freshman.' 'OK, sounds good.' Every once in a while obviously there is a question or two depending on what it is, if it would cause chaos or something. But other than that, just allowing people to do that. They are pretty good." 
He tries to be supportive of his teachers trying new things and he feels they are more likely to try new things because he has worked hard to build trust and a positive culture at the school.

Becky recognizes that their school has the benefit of a lot of time dedicated to PD. "Every Wednesday the school has early release time for two hours of PD so we are fortunate in that way." As an administrative team they make every attempt to structure that PD time to meet the needs of all teachers. Taking advantage of the time that they have with their teachers, Becky states that they have changed the direction of their meetings. "The faculty meetings are very PD focused, so it's very seldom they just sit and get, it's much more."

\section{Interviewee \#4: Alicia}

Alicia is the principal of a suburban elementary school. She has been at her current school for eight years. She has approximately two hundred seventy-five students and twenty-eight faculty members in her school. She says, "I just have a great staff that we talk about making improvements and they are really active on learning. I would say that they are a fairly younger staff, they probably range in age, well, fairly young, they are all in their tenth year teaching, ten and fifteen years teaching. Even some of the people who are older, had taken a break, raised kids, and even my older staff are only about fifteen years in." She also recognizes "Most of my teachers have a decent amount of seniority that they wanted to be here and they chose to be here." She is the sole evaluator in her school, "being a small school, I share my art and music and librarian, and I do theirs as well." 
Alicia identifies her administrative style as being supportive of her teachers. She tells her teachers, "Let me know if you need more time. I can arrange that. I'll cover your class." She recognizes the dual benefits of helping teachers by covering their class for them. First, it provides them the time they need to work with others or plan. Secondly, it also allows her the opportunity to be in her teacher's classes and get to know her students and classes better, as well as identify things her teachers might need. "Being in the classroom that much I have a better idea of what they're doing and so I'm apt to say, if there's an article in educational leadership or education weekly that really pertains to what they are doing, I'll actually photocopy it and hand it to them." In addition to articles or ideas, she also notices, "if a supply or material was missing and I see them trying to work around that in their lesson I can say, 'you know we can order that for you, let's order that, it looks like that would make your life easier."

Alicia describes herself as someone who focuses on sharing new ideas with her teachers. "My staff would tell you I'm definitely a reader, and I'm definitely a thinker, I constantly am asking questions, thinking about what we might want to do next." She also comments, "I read a lot more research than they do, I read a lot more non-fiction than they do." Therefore she is always thinking of new things to try and likes "to plant little seeds and let them grow." For the upcoming school year she has "already put some seeds out about what could happen for professional development." She feels her teachers are very comfortable coming to talk to her. "I would say just about everybody on my staff shows up at my door to talk to me about what's going on at least every other week, if not once a week." She recognizes the importance of open communication and that her style of "leadership contributes to a less stressful environment." 
Alicia identifies time as one of the supports that the teachers at her school have. "I would say this district, specifically this school, has the support of time. In the sense that these teachers have a lot of time to work together." Some of the times she identifies include, "common planning time once a month. Then we have faculty meetings, once a month as well. Then we also have 4 professional development days throughout the year." Another time that her teachers have opportunities to work together is lunchtime. "Everybody here has the same lunchtime as well. They have fifty minutes while the kids have lunch and recess." At her school she identifies that she also has "a school improvement team meeting once a month that I would say about $80 \%$ of my staff regularly attend."

Teachers have also given their own time to Alicia on occasion, "One time I ran out of time and I was like, 'I'm sorry I didn't get to that at the faculty meeting and I wanted to share that.' And they are like, 'Why don't you come to lunch today and share, finish it then? Why don't we finish during that fifty minutes?' So they are very willing to give time, and I respect that, so I wouldn't take advantage. I don't usually go into the faculty room during those fifty minutes, that's their time."

\section{Interviewee \#5: Garry}

Garry is the principal of a suburban elementary school. He has been at his school for twelve years. He is the only administrator in his building and has approximately three hundred students, twenty teachers, and thirty faculty members at his school. He states about his school, "It's a nice population. Really highly involved parents, in a positive way." He recognizes the importance of involved parents, "Standing room only at open house. Parents, grandparents, I mean everybody comes, you know.” He does not limit 
who can come to events, so they often have over six hundred or seven hundred people at school functions. "So in a lot of ways we are blessed with that. We can't keep people away. Which is great."

Garry is the only evaluator at his school and is responsible for evaluating all teachers as well as teacher aides and secretarial support staff at his school. "Obviously the first couple years we had everyone in the building going all at the same time." "Specialists like our school psychologist and social worker, they're done through central office. I can aid, I can add info, I can add my perspective to it but that's been a little bit of a lighter load."

Garry identifies his administrative leadership style as one of working with his teachers. When he stated the evaluation process he remembers, "I kept projecting, 'we're in this together. We're learning this together." When he first arrived at his current school it took some time to get everyone onboard with his philosophy. "I spent a good two years getting everybody, my tag line was 'everybody's welcome." He emphasizes the importance of being supportive but also holding his teachers accountable. He focuses on working together and supporting his teachers, but understands there are times he has to say no to them. 'I'm generally thought of as the nice guy, but then I've had to be not so nice. It's actually like when you're parenting, if you yell all the time, who listens? That's kind of my style so when I finally get upset and when I have to put somebody in their place, or I disagree with them, or they need to know that I have final word, they listen." When he does have an issue or needs to speak to someone, "it kind of reverberates through the school. Then they all have lunch, 'He did this' or they say 'He was so upset, but you know he was right."” 
Garry says that when something new gets introduced in his building, he works hard at keeping positive and focusing on all the things his teachers are already doing. "You are hitting a lot more than you think. That was one of the things we kept trying to tell people early on, even with the common core transition, it was 'You already do this, it just looks different; it's just worded differently. Don't get so uptight and stressed."' But even with his encouraging words people were feeling the stress. "We had to work on that a little because I felt the stress level in my building, I was like, 'Come on guys, we always do well. Just keep doing what you're doing. We are just redefining everything, just reorienting yourself to a new system. It's no big deal."”

Garry also focuses on maintaining a positive attitude with his teachers. "They would say to me, 'Why aren't you so stressed about this?' What are you going to do? You can't. I can't. I'm not going to walk around the building projecting my stress. Because then you're stressed, the kids are going to be stressed. You're projecting your stress on them. So everybody just needs to take a step back and calm down a little bit." He tries to remain positive and encouraging.

Garry has limited time devoted to full day PD. "Unfortunately, full day PD is at the beginning of the year, one day at the very beginning and we have one day in the spring." They also have "some PD after school, some of it is voluntary PD." In addition to the after school time "beyond the voluntary, we're lucky, we do have one faculty meeting per month, and we have one curriculum based meeting per month and I have that flexibility." Garry also has some flexibility to adjust the time and then break up the curriculum meetings. "That curriculum meeting, because we're an earlier school, we can call it before school. So I have the flexibility to make that either grade level or curriculum 
for school wide types of things." He has two monthly meetings for covering PD or other topics that he can use at his discretion. Garry's PD time during his faculty meeting and curriculum meeting times is not always whole school. "I would say some whole school, if I had to do percentages, it would probably be $50 \%, 25 \%, 25 \%$ break up of PD whole school/ small group/ individual."

Garry recognizes the importance of electronic communication saying, "I do a lot of email. We have an internal newsletter, an internal list serve. I'm constantly sending teachers things. I review it at those faculty meetings." He also knows how important it is that he be visible throughout his school. He is always willing to meet with teachers, or parents. "I think we meet all the time. I'm constantly booked. I never say no."

Garry feels he gets support from his superintendent and other administrators in his district. He states, "My superintendent is really good about giving us autonomy." There is also another same grade school in town he works with. "The nice thing is that I work closely with the other school's principal. We've been together right along, with each other the whole way. So whatever her faculty is doing my faculty is doing and vise versa." He also feels supported by other administrators in his district. "We do meet as an administrative council once a month, sometimes it's twice because there is always a lot to talk about." All of the administrators in district "try to be as consistent as we can with PD.”

\section{Interviewee \#6: Zachary}

Zachary is the principal of a suburban middle school. He has been at this school for twelve years. There are approximately 950 students, one hundred ten teachers, and one hundred forty faculty members at his school. He is facing declining enrollment in the 
past twelve years since he arrived at the school. As his "numbers continue to decline, it makes it harder to run the building." As a fairly large middle school, they "offer the full range of sports, we have a lot of extra curricular activities."

He has one assistant principal at his school and they split evaluations. Zachary and his assistant principal "do the bulk of the observations. We do all the evaluations and the beginning, middle, and end conferences." There are two teachers who assist with observations in their building. "They do one unannounced, so what will happen is if we have a concern about someone we may ask them to do two and I may do one. What happens is they get different view points." Between the two building administrators, "we each take on a certain amount of the caseload. The assistant principal will do a couple more than I do, but I do all the non-certified. We pretty much divide it. Then there are some people like school psychologists, social workers, and self-contained teachers that we don't do, special education does that."

Zachary tends to have his assistant principal "do more of the non-tenured teachers and spend more time in there. I tell him I don't care if you need to do a fourth and a fifth observation, I don't care if you are doing three that don't wind up in EPSS [Educator Performance and Support System] or you're doing walk-throughs and you stay there. In the first six months of hiring someone I need to gauge, I need a good gauge. I'm not worried about classroom management. I'm not worried about stuff like that that much. I'm worried about can they teach, do they love kids, and are they passionate about it?' Zachary's assistant principal at his school is focused strictly on teaching and learning. The assistant principal, "does nothing that is not teaching and learning. Absolutely nothing." Zachary states his teachers "look at me as the moneyman, the 
political guy, when there's a jam who's going to get them out of it. When there's an issue with a parent, they come to me. When they have broken furniture and they need it fixed, all that stuff."

Zachary identifies himself as a leader who encourages his teachers to take risks and make mistakes. While he pushes his teachers "hard for results." He also tells them, "if you fail I will be the first one in front of the school committee saying, my fault." $\mathrm{He}$ encourages his teachers to try something new. 'Let's try it. If it doesn't work we'll go from there." He wants teachers to be willing to try new things and says, "I will take the blame for the failure. Then I say we have to learn from it. What can we do differently?" He encourages his teachers to try new things and learn from their mistakes. "What I tell people all the time is we can't keep making the same mistakes. If I have a teacher doing the same thing over and over again, and they come in and say, 'You said we could fail.' But we can't be stupid about it. If you keep doing the same thing over and over again and you get the same results, if you're expecting a different result, that's the definition of insanity." He wants his teachers to be willing to try new things and if they do not work, he encourages them to learn from their failures.

Zachary also identifies time devoted to professional development as a positive support for the teachers at his school. "Once a month we have an early release day." In order to get longer meeting times "we took our early release and our staff meeting and we put them on the same day." This provides them with three hours of PD once a month. They set up their PD plan for the year at the beginning of the year. The "assistant principal meets with our content leaders and sets the PD calendar up and we give it to them in September for the year." Zachary says they have shifted their PD focus as well. 
At the beginning and the end of the PD meetings "we will meet as a staff very quickly." Meeting at the beginning sets up the activities for PD. Coming together at the end of the meeting is when "a lot of the celebrating takes place. That's where sunshine will say who got married and stuff, and it's a time where they're congratulating each other or just say things. We give them that time if they need it. And then if we're done early we send them home."

Zachary emphasizes the importance of electronic communication to share information with his staff. "We send out a weekly staff email, that is produced by a teacher, everything is sent to the teacher. It's organized. It's sent to me, and then I send it out. $95 \%$ of our staff meeting is in that weekly update." This allows him to focus more on PD during meetings instead of dissemination of information.

Zachary recognizes that after many years at the same school he "can spend more time on evaluations" because he has some school supports in place. "I have a School Improvement Team [SIT] that is incredible. A PTO [Parent Teacher Organization] that anyone would die for, a PTO that I do not get one headache from; zero amount of work, and every meeting they ask, 'What can we do for you?' [I have] a School Improvement Team that actually makes changes and makes recommendations that are good for our school." He also states that he has a good working relationship with the teachers' union at his school.

\section{Data collection, and timetable}

In this phenomenological study, I collected data through face-to-face interviews with participants ranging from forty minutes to one hundred minutes long with the average time being seventy minutes. I conducted the interviews over a period of two 
months, from July to August 2016. In addition to the interviews, I asked participants to share documents related to professional development they conducted at their schools as well as relevant evaluation documents. I used a general interview protocol (See Appendix D) to guide the interviews and ensure consistent topics were discussed in all interviews (Moustakas, 1994). Interview questions were open-ended and I encouraged participants to share their experiences as a way to allow them to expand on their ideas. I asked follow-up questions using the interviewee's own language, for clarification purposes or to expand on ideas presented. (Moustakas, 1994).

I also asked participants to provide artifacts of their practice related to support and/or evaluation, as collection of documents is an important way to triangulate and validate data collected in an interview (Merriam, 2009; Patton, 2002). Related to support, administrators provided calendars, activities, notes, outlines or presentations, common planning time (CPT) schedules, agendas, and/or minutes. Related to evaluation administrators provided notes from observations, conferences, websites, feedback provided, sample PGGs (Professional Growth Goals), schedule of evaluation dates and expectations for the year, as well as other documents that demonstrate their role evaluating teachers.

The quality and consistency of documents varied for each of the interviewees. Only three of the administrators provided me with documents, and each of those were emailed to me after the completion of the interview. Some administrators do not routinely collect and keep copies of PD they have delivered while other administrators have an electronic website that was shared with me where PD documents, videos, and other resources were stored. One administrator provided me with a generic PGG that all new 
teachers in the building must use that addressed the building wide expectations at that school. Most documents reinforced conversations and comments that were made by administrators during the interviews. Much of the information shared was access to electronic documents used by the administrators.

\section{Data Analysis}

Each interview was audiotaped on my iPad and then I transcribed each one. Once transcribed, all identifiable information was removed and saved as a clean copy.

Interviews were analyzed in several phases using a modified from the Van Kaam method of analysis of phenomenological data (Moustakas, 1994, pp. 120-121).

The first step in analysis was bracketing. During bracketing I read through the entire interview and identified phrases or statements in the interview transcript that were relevant to the topics of evaluation, support of teacher's growth, and administrators balancing these dual roles. I then re-read through the phrases and identified themes based on those phrases. I then grouped the direct quotes by theme and saved them in a separate document.

I then wrote a summary of the themes, using direct quotes from the interviews and shared them with the participants via email to ensure accuracy (Moustakas, 1994). Only one of the participants responded to the email with a brief reply of "Looks good." I resent emails to participants but received no further replies.

Once themes were identified individually for each interview, I then looked for overall themes that were present in all of the interviews. Each individual interview was color coded to identify the speaker and then all direct quotes were collected based on the identified themes. I then created a textural synthesis for each theme that included direct 
quotes from each of the participants used as supporting evidence throughout (Moustakas, 1994).

\section{Validity}

There are several types of validity that are important to address within a phenomenological study. Member checking or respondent validation is a common form of descriptive validity used in phenomenology. Member checks are built-in and conducted throughout the data analysis process. After the individual interviews were conducted and descriptions were created, these descriptions were shared with participants, allowing them the opportunity to provide feedback on the intent behind the words and meanings from the interview ensuring interpretive validity (Creswell \& Miller, 2000; Merriam, 2009; Mertler, 2016; Moustakas, 1994). Individual summaries of the themes for each participant were shared with the participants via email, but only one replied to the email.

A significant part of the phenomenological approach to conducting research is the concept of researcher reflexivity. This is the act of taking a critical look at my own stand on the given topic. Phenomenology formalizes this through the process of epoche. Before conducting the interviews, I engaged in the interview process myself and had a colleague interview me. This helped me to address the concern of researcher bias by actively identifying and bracketing my own feelings related to the topic of teacher evaluation and support (Merriam, 2009; Moustakas, 1994). I also kept a journal of field notes throughout the process in which I logged all of my contact with interviewees and wrote about my impressions after each of the interviews. 
I also attempted to address the concerns of evaluative validity by repeatedly reviewing the themes and original interviews to ensure consistency between themes and participants' intent (Moustakas, 1994; Creswell \& Miller, 2000).

The concept of generalizability, or transferability, is somewhat limited because a qualitative study by nature looks in depth at a small group of participants. Therefore, generalizability lies with the level of description with which the data is presented. In Chapter Four I will share with you the themes identified through the interviews and quotations from the interviewees to support those themes. Through this description, individuals can use the information shared to make connections to their own situations (Creswell \& Miller, 2000; Moustakas, 1994; Merriam, 2009). 


\section{Chapter 4: Findings}

\section{Introduction}

The purpose of this qualitative research study was to explore the perceptions and experiences of administrators tasked with supervising their teachers' professional growth as well as evaluating them using the RIDE created educator evaluation system. Data was collected through phenomenological interviews with six RI administrators. The data collected through the interviews helped in addressing the proposed research questions that were designed to better understand the supports provided and the challenges faced by administrators implementing the RIDE educator evaluation system.

The data was organized into themes for individual participants and then larger themes and categories that addressed the ideas and experiences of the participants related to each of the four research questions. Themes and experiences are reported out to explain the general ideas and similarities of experiences for all or most of the participants through the interviews. Data will be reported out for each of the individual research questions.

RQ 1: How do RI administrators who are responsible for both supervising faculty professional growth and conducting educator evaluations support the growth of their teachers?

Research question one looks at administrators' strategic use of professional development supports. Administrators used modeling and transparency to help demonstrate best practices for their teachers as well a providing differentiation and personalization throughout PD activities to help teacher be engaged and invested. They have also attempted to make use of social learning supports for their teachers through 
PLCs or focus groups to allow for choice. Administrators also discussed the various supports they provide for new teachers and improvement plans for struggling teachers.

\section{Strategic Use of Professional Development}

All of the interviewees discuss ways in which they provide support for their teachers' professional growth. They mentioned how they make careful use of the PD time and intentionally structure their meetings to maximize the impact of the PD provided.

Administrators discussed some of the PD topics they presented in recent years. Some of the PD topics that Zachary and his administrative team have focused on include blended learning, specific teaching and learning strategies, how to use devices to move into the twenty first century, as well as evaluation specific PD.

Margaret offered PD on topics such as growth mindset, blended learning, and the evaluation system among others. She is always pushing herself and her administrative team to be involved in learning about new professional development activities and ideas. "I really wanted to think about professional development, I wanted to think about educational leadership and culture, all things that kind of integrate with one another."

Alicia states she is "definitely the person who is driving the professional development" at her school. She recently focused on PARCC [Partnership for Assessment of Readiness for College and Career] data analysis. "Last year I did a share out on our PARCC data and then I did released items and we took them, as a staff, and we talked about that. And then we did a 'what's working' comparison to what are we doing now that would meet this. Then what would we need to add to raise the rigor. That was over a series of three meetings. The year before that I did a lot with growth mindset." Alicia also works with her teachers on setting goals with students. After reading an article 
about eight effective practices in schools, "we talked about which ones do we see at our school and which ones do we see, but we need to increase."

Garry states, "We try to stay cutting edge, we try to stay on top of things." Some of the topics he has covered in recent years include growth mindset, differentiated instruction, blended learning, flipped classrooms, inclusion, study of the standards for common core, training for a new math program, intervention programs for reading and math, and training for science kits. They also use meeting time to discuss the evaluation.

Garry uses his "monthly faculty meetings and curriculum meetings to try to individualize." He structures his meetings so that he can have each grade level meeting on different days. "I had different days of the week, which I had to get permission from the union for. Our meeting day is Tuesday morning before school, but if somebody would agree, I would have maybe fifth grade Tuesday, fourth grade Wednesday, and third grade Thursday. And then we would individualize it for the grade level. That was really nice."

This is the third year that Becky and Thomas have used an essential question to guide their PD. They set an essential question at the beginning of the year and spend that year working on ways to answer that question. PD for the year focuses on the essential question and teachers are given personalized learning time to also answer the question on their own. Some of the topics they have discussed include technology, personalization, and data usage. Becky recognizes that some of the teachers are still "grappling" with the first or second essential questions, while others are ready to "dive into this next step" of a new topic, while others still want to "go deeper into" one of the previous topics. Becky encourages teachers to be involved in the planning of PD. "Actually this afternoon I have 
a group of teachers coming in that went to a training recently and they kind of helped us to craft what the PD will look like next year a little bit."

In addition to the topics they discussed during PD, administrators also talked about the ways in which they structured their PD sessions including their own active involvement in PD sessions and the use of protocols.

Thomas identifies autonomy as a positive about the professional development at his school, especially for their most effective teachers. Thomas and his administrative team focuses their PD "on the most effective people and we make decisions based on those most effective people so I think it's had this real impact." He identifies that the teachers in his building "used to be so frustrated because they weren't" growing. With his new PD system, he targets those teachers and he recognizes, "we are at least growing those sort of highly effective, totally motivated people."

Margaret recognizes the importance of providing teachers with the support necessary to be successful with new initiatives and topics. When introducing a new topic or skill for teachers, she states, "I will never, ever tell you you have to do something without telling you, showing you how I can do it, and giving you the supports to learn how to do it for yourself." Margaret ensures that her entire administrative team is supportive of teachers. "We, the administrative team, are trying really hard to make sure we don't ask anybody to do anything without providing them the stuff and showing them first that we are going to do it too and that we are rolling our sleeves up and we are going to do it together."

Margaret also finds that the use of protocols during PD meetings helps keep people on task and professional. "Protocols work like a charm. Nobody can go off on 
their own little rant. You have to stay with the protocol, and when I only have forty-five minutes or fifty minutes to do things, they are beautiful." She has established and employs protocols and "if teachers are not going to be professional about it I am going to put a pin in that thing and move on to the next person."

Thomas also finds "the use of protocols to professionalize the dialogue and the culture when teachers are meeting together" is helpful.

\section{Modeling and Transparency}

Modeling what is expected of teachers is something that several administrators identified as important. Margaret states, "What I feel we have to do is modeling it. Modeling it. Modeling it, and living it." Zachary states he is not active in planning PD activities at his school, as that is the responsibility of his assistant principal. But he is an active participant and believes it is important to model the engagement he expects from his teachers. "I tend to always go to the beginner technology workshops. And I think that's good because we have a lot of teachers that will be there. But they see me in there learning with them, they see me struggling with it." By participating with the teachers in the training he models active engagement and states, "they see that it's real and that they aren't the only one struggling, but they also see that we need it."

Becky identifies that it is very important to present PD for teachers modeling best practices that teachers can than use in their own classes. "When things come up that we have to get out to the faculty, I'll structure the PD meeting so that it's like a station rotation model or it's blended in some way. Watch a video. I put it on edpuzzle [electronic tool for sharing videos for educational purposes], so even if the topic of the faculty meeting is low in content, I'll structure it so we can model some sort of best 
practice or way to deliver it so that it's not just the sit and get. It's a neat kind of relationship in that way." Through PD activities Becky and her administrative team model expectations they have for teachers in their classrooms. "The students should know the question ahead of time, just like you know your essential question at the beginning of the year, this is what you are working towards, how you answer it can be different." As an administrative team "we are really trying to model that with them." Administrators also felt it was essential to be transparent in their actions and even spend time explaining and reflecting on activities to help teachers understand and apply some of the practices they used. Margaret says she sets high expectations for her teachers, but is also transparent about her expectations and priorities. "I'm really honest about that with people and they really know that my priorities are set." Alicia recognizes the importance of being transparent as well. "I'm very transparent. No one has ever come in here [her office] and heard something that they were surprised about hearing." When meeting with teachers, she is clear on her expectations. Transparency is something that Thomas identifies as an important focus. "I think that we are pretty transparent about why we are doing something during PD." He finds "that kind of transparency builds some trust." Becky says they also focus on setting clear expectations. "What does it mean when you get there? What does good look like?" She and Thomas have created a document for their staff entitled: This is how we do it. "One of the things we realized with the faculty is that they want to know what to do." She comments, "It's part of that transparency again.”

Becky realizes that they need to take the next step with their professional development for staff. She states, "the next step for me professional development-wise, is 
being transparent about the process and giving teachers time after to reflect on the process." For example, the teachers "went through this station rotation model, but only a third of the people saw the model and then used it immediately in their classroom. Because they thought, 'Oh, that's a great strategy. I get it. I get why she put the chairs in this way in this particular room and why she structured it.' I've always done things in that way, because as soon as someone structured it in a PD meeting [for me], I would take that to my room. But now I'm cognizant of the fact that that was me. We just need to be much more transparent and open about the process and make time at the end to reflect." Becky states they try to "talk about the practice that was used, or the strategy that was used to get to that content. That's going to be more of a focus this year and really giving time for teachers to be able to reflect on that afterwards. How did that model work? What worked about it? What didn't work about it? To move them to the next step."

\section{Differentiation and Personalization}

When asked about how they differentiate PD for their teachers, administrators interviewed discussed various strategies they use. Margaret discussed that during one full-day PD, she created a differentiated Bingo card activity. There were activities, like on a Bingo card, for the different content area teachers to choose from. But there were "some non-negotiables right in the middle and whatever you do [has to be] on that Bingo card." Staff could choose the activities they wanted, while Margaret was still able to get certain things completed. This helped all staff remain engaged and invested in the activities and they were all able to be "working at their own pace."

Margaret also recognizes that when differentiating for teachers, some teachers need to be left alone to do their work. "So that's part of my support too, leaving you 
alone. Letting you just do what you do best if you do best. You don't need somebody, some chin on your shoulder all the time. I feel like sometimes you just need to sit and reflect without having somebody there.” By providing differentiated supports, Margaret finds that, "people accept what we are doing."

Margaret also notes, "even if you ask what they want to do for PD, they often will not have their own suggestions, because it's too obtuse, I think, so when you give the three choices maybe that's better, like station rotation type thing." She finds that providing teachers with a few options and then allowing them the opportunity to choose works best. "I would say, these are the main ones, if you have something different, you have to explain what you are doing and why. That's it. So, most people pick the main ones." This allows teachers to personalize their learning with support and guidance from administration.

At Becky's school they also spend a lot of time differentiating PD for staff. Each staff person has eight hours of personalized learning time. For the past several years, the administrative team has developed an essential question that teachers need to focus on for the year and teachers are expected to use their personalized learning time to answer the essential question. They are encouraged to answer it in any way that works for them. When teachers ask about an outside conference or PD activity, Becky states, if "you think that PD activity is going to help you answer the question in the end, or help you out in your classroom, then go. If you want to go to, whatever it is, as long as you log that time." After completing their personalization hours, "they reflect on their time and how it supported them as a learner ... and then I keep track of that." The personalized learning 
time lets teachers "do their own thing, as long as it helps them" answer the essential question.

In addition to the personalized learning time that the teachers have, Becky and her administration also organize PD meetings to address the essential question for the year. Whole-school PD time is often differentiated for teachers. "If we have the full staff on PD days, those days are usually around choice. Often we start with an assessment or a pre-assessment and then there are a number of sessions that they can choose from based on their pre-assessment." Becky states, "we offer choice many times. We will use choice boards and things like that for them to be able to have some choice around where they're at because the goal is we don't want you sitting for something that is a waste of your time, that's not what we're here for." Becky also uses a badging system to help with differentiation. "The other thing we are trying to do as far as differentiation goes is a badging system. I just started a professional badging system for these things that we said are important."

Thomas encourages personalization for his teachers for PD. "Because it's so personal. If we are talking about personalized learning, it's personal for that individual teacher too." His administrative team is "trying to create pathways for teacher and teacher leaders on some kind of continuum." The goal would be as teachers move to a certain level, they would allow teachers to have, "full autonomy in your classroom and make decisions the way you want to around assessments and this that or the other thing. We just haven't figured that out."

Garry also tries to differentiate and personalize PD options for his teachers based on their interests and needs. "I do have pockets of people who know what they want and 
we've tried, as the evaluation tool changed, to individualize things a little bit more. We really do try our best to ask many times and survey to kind of figure out what do you want and what do you think you need. So we have individualized more the last couple of years." Garry has tried to give teachers more of a say in their PD, but, like Margaret, has found that they sometimes struggle identifying their own needs. "They never really felt like they had a say in their PD, like what their needs were, but then we had to kind of gradually ease into that, because it's hard to say what you need. And sometimes even if you want to, you don't know."

Zachary also tends to do a lot of differentiation at his PD sessions. "It tends to be like a playlist or a menu. So they are going one of ten places or one of eight places." They divide up the time differently. "Sometimes we do an A and a B. Like 1:00-2:00 will be one activity, and 2:00-3:00 will be another, so they're getting two. Or sometimes what we will do is one activity and then tell them the following month they will have other options. And sometimes from 2:00-3:00 we will give them an hour to now produce work based on what they've learned." Another way Zachary splits up time is, "Sometimes we let them go back to their own rooms and do their work and sometimes we have them work right there and the lead person or people now become the facilitators." Most of their PD is done in small groups. "We will do whole group if we absolutely have to. We try not to because there's teachers, school psychologists, social workers, plus teacher assistants are there for part of it sometimes. You're talking, first off, on any given day one hundred twenty people and some of them aren't classroom teachers." They try to have a variety of activities that pertain to individuals or groups of people. 


\section{Social Learning Supports}

Several administrators identified the importance of having their teachers work together to take advantage of social learning opportunities. Some of the administrators use PLCs (Professional Learning Communities) or focus groups to help their teachers select topics of interest to them and work with other like-minded colleagues.

Margaret combines differentiation with social groups to help teachers work together on topics that are of interest to them. She typically sets focus groups for the year and teachers choose the focus group they want to participate in. "So they can choose whatever they want. If they don't want to do one of those three focus groups, they can then choose something outside of that. And say to me, 'I'm interested in X, Y and Z...I want to teach and I want to use that for my kids in my classroom,' I say, 'Wonderful, thank you, awesome." While providing several focus groups she also allows teachers to choose something outside those focus groups, if they have something they feel is relevant.

Margaret also set up model classrooms with some of her teachers when she introduced new technology. "This past year we had seven model classrooms in the high school." This helped other teachers see what was happening in their peers' classes. For the model teachers the administrative team asked, "What are you doing? How are you modeling it? Do you mind teachers coming into your classroom? And you know some of the people were clamoring and others were like, 'I'm not all that comfortable yet. Can you give me time? Can it be like, yes they can, but can it be third hour on this day?' 'No problem, whatever you want, that's what we are doing." She works with the model teachers to help get other teachers in to observe what is happening in their classes. 
Becky and her team provide choice workshops as well as create Professional Learning Communities (PLCs). "We are going to form PLCs this year, which will be different around each of those areas of personalization, blended [learning], or data use. So we'll see. It's quite intricate, I guess, and it's really personalized for the teachers and then at the end of the year we have a sharing event." They are also offering more choice with the PLCs this year. "This year will be more choice in that they will be able to decide on their PLC and within those PLCs they are going to decide small groups based on the broader topic we differentiate a lot based on choice and where people are at."

Alicia also identifies the importance of teachers working together. They have common planning time. "All of my teachers have forty minutes of common plan time. Four times a week they have forty minutes of common plan time, plus that fifty minutes that the whole school has lunch together." Because of the time that her faculty have together, "we have a lot of networking that happens in our school. It's a really nice small school in that regards."

\section{Support for New Teachers}

The administrators interviewed use different programs and supports for new teachers at their schools. Some have formal mentoring programs established by their districts while others try to offer informal supports for teachers on their own at the school and individual teacher level.

Alicia has a district-wide mentor program for new teachers. "We do have a formalized mentor program. So they meet once a month, I think. Once a month for the first year, but then after the first year you really are expected to build that network, so it's a catch in that regard." 
Garry's district also has a mentoring program. "We've got a really solid mentor program. And some if it was through induction, the training and what RIDE provided. We've always had a mentor program, which has always been a positive thing." $\mathrm{He}$ recognizes that new teachers in his school are fortunate in that, "they have had real strong veteran partners, so they [the veteran partners] have really taken over mentoring, so that's really great." In addition to the formal program in his district, he also has more of an informal mentoring partnership. "Free of charge my fourth grade teacher supported another teacher willingly. Gave up a lot of time, volunteering her time, to do that.” Zachary's district has "a mentor program in place" for new teachers. They have a district-wide mentor coordinator "but the person is part time doing that and part time something else." He states, "I meet with her quarterly on each new employee and I tell them how it's going. I will get into concerns with the mentor coordinator. Then I meet with each mentor. The mentees I don't meet with unless they ask to or unless we have a problem." He recognizes "it's a top down approach." He meets with the mentors and expects the "mentors will bring issues or problems to the mentee's attention." Zachary mentions that, in addition to the formal mentor program, "We try to give new teachers a better schedule. We try to make it lighter if we can. We try to make it more ideal. We know certain classes are just killing new teachers so if we can avoid it we do. If we can't, we can't."

There is no formal mentor program at Becky and Thomas' school. Becky states, "Our department heads really do work almost as a mentor. Speaking of, one of the things we don't have here is, when induction went away, now there is nothing district-wide to support new teachers. We don't have mentoring." The department heads at their school 
provide supports for new teachers. "Our department head structure works well. Yes, we [administrative team] are here to support teachers, but we have department heads." She also notes that amount of support given by the department heads varies depending on the department. "I think about one department who has had a lot of new people in that department; it is tight. We don't support those teachers; their colleagues support each other. And so having a school that does that for each other is really important, and we're lucky to have a culture that does that. Other departments? Not as good. Not as welcoming."

Becky and the administrative team focus on other informal ways of helping and supporting new teachers. "We give our new teachers a duty off. They have that time so they can go into a colleague's classroom, they can sit with one of us administrators, make appointments with one of us, have a little extra time to kind of dig into what they want to do." They also split the roles of the administrators when it comes to new teachers and the evaluation. Becky states that the principal, "would be the evaluator for new teachers, so that would kind of put me in a role that is not as threatening maybe, so that I could go in and support." But not all new teachers take advantage of those supports. "There were two new teachers who took me up on it this year where I said, 'Happy to come in, give you feedback, go through the eval with you. I'll focus on anything you want me to focus on.' So to be able to give them that support. And we had, one teacher was really good, one teacher who was always inviting me in. 'I really want to know what you have to say, give me some feedback.' So that allowed me to have that relationship with him. And then the principal could be the heavy when it came to the eval, to try to take a little bit of that off. 
So we did that. But some teachers were more open, some newer teachers were more open to it and wanting to do that than other teachers."

\section{Support for Teachers Needing More Support}

Whereas administrators discussed many different types of professional development, and having opportunities for whole-group and individualized PD, in discussing support for teachers who struggle, administrators turned to the evaluation system. Some of the administrators mentioned making use of the improvement plan included within the RIDE evaluation system to help address identified areas of concerns with teachers who are struggling. For example, Margaret uses the evaluation system to help teachers by providing supports where she has identified issues in the classroom.

"There have been a couple of years now where I feel there has been some unprofessional behavior going on in that particular classroom. Anyway, he is going on an improvement plan, because you can put anyone on an improvement plan. He does not have to be observed as far as his SLOs [Student Learning Objectives] and stuff like that but he's going to be observed as far as what's going on. It's the first time I'm doing this [new RIDE improvement plan]."

Zachary similarly discussed the evaluation system improvement plan as helpful for dealing with teachers who struggle, citing that his district has a formal system for improvement plans for teachers. "Once they are on an improvement plan it involved the union president and the superintendent. They are pretty hefty plans. They are pretty aggressive. And we tend to meet at least quarterly if not monthly. And when we do that it's the superintendent, it's the union president, it's the teacher and it's myself. And you know, they agree to A, B and C and before they leave the meeting I always say to them, 
'Do you know you are agreeing to read in this book, you're agreeing to go to this conference, you're agreeing to do your lesson plans this way. When we meet next month, chapters one through four needs to be summarized, this conference needs to be registered for. And this lesson plan in this format, I need evidence to show you are doing it."'

While Zachary recognizes the importance of improvement plans he admits that he tries to avoid them because, "they always feel like they are more punitive." In his district they used to have a policy “that you couldn't stay on an improvement plan for more than two years. Then the second year if you are on an improvement plan you are terminated regardless of anything. But that is no longer in the contract." If they have a teacher who is struggling, they "make sure the assistant principal is not evaluating them, and we put him on them. He will work with them. He will meet with them once a week. He will go in and observe their lesson. He will go in and give feedback. He will co-teach with them. He will design a lesson for them. He will teach a lesson for them. And when we do that, it's really easy because it's not punitive because he has nothing to do with the evaluation. And then I tend not to talk to him about that employee. And then, the hope is you see the growth."

Alicia also discusses thinking outside the improvement plan to help teachers who needed extra support. Alicia says, "In the beginning [of conducting evaluations] there was a couple of times I went in and I didn't write up a lesson. I often give everybody one chance to not to have that written up; that's just a conversation. But if it happens again, I feel like that's where the evaluation system could have strength, if you really need to evaluate somebody who was not having a lot of success in their grade." Although Alicia does see a strength in the evaluation system, helping with some teachers, she emphasizes 
that, "I'm not in there to not support them, so when they need a lot of support, I don't write up that lesson. I work with them for the first time.” Therefore, she gives teachers an opportunity to improve before it gets written up. 'I'm not saying I've never written up a lesson that was not successful. But the very first time I do not do that."

$\boldsymbol{R Q}$ 2: How do administrators set the stage within the context of their schools to be able to supervise teacher growth and evaluate their teachers?

In order to be able to supervise teachers' professional growth and conduct evaluations, administrators need to ensure that they have laid the right foundation for their schools. All six participants discussed the importance fostering a positive culture and climate at their schools. They also discussed the significance of developing trust, building relationships, and recognizing faculty. The development of a strong culture was something they employed to assist in implementation of the educator evaluation system.

\section{Climate and Culture}

\section{Trust}

The administrators interviewed emphasized the importance of developing a trusting climate in their schools and discussed various ways in which they built trust. Thomas talked about trying to build trust, saying, it's "relational, it's personal, just being out there, you know, trying to be supportive." One of the ways he works on building trust is by the PD and other supports they set up for teachers. "We are trying to create this, sort of, non-threatening information data that will hopefully spark some kind of growth and interest in the stuff we are doing with PD and everything else." Another way Thomas builds trust is by addressing with teachers right away any issues or concerns that he receives. "Whenever I get any kind of complaint about a teacher, an email or whatever 
else, I share it right away. They were kind of freaked out about that in the beginning. But in the past I think the administration used to save up ten emails and then come after somebody, that type of thing."

Garry notes, "the trust overall, I have to say, I'm really lucky that the building as a whole has a really great, incredible supportive culture," and that the school has an “overall environment of caring, compassion." He recognizes that it takes time to get to know the people and develop trusting relationships. "I had five or six years of building trust with this faculty, and it was pretty much the cluster, the chunk of faculty staying together. They were already a pretty tight-knit group, so I was the new person on the block. So I built a lot of trust." Garry's whole school works together on developing a positive culture. "We worked a lot on that whole school culture piece." And because of the work that was done on the school culture, Garry feels that the teachers trust him and move forward with him on new initiatives. 'I think that's helped, all these new initiatives, kind of fall back on trust. You know everyone generally likes each other too, we are a family, we fight, you duke it out a little bit and then it's over, so we've been able to kind of sustain that over the years."

Becky also realizes that all of the administrators in her school work on building trust by "being visible, and personal" when working with teachers. They also have the attitude of "encouraging teachers trying different things and it's a no-risk, just try it. So what doesn't work, not a big deal." This helps teachers to be wiling to try more things and take risks while trusting that the administration is not out to get them.

The importance of culture is also reflected in the success Zachary has doing evaluations at his school. He identifies that "what principals have to do with the whole 
evaluation system is make sure they set up a culture of, 'we're going to make mistakes, we're going to learn from our mistakes, we're not going to hammer you for your mistakes.' What I tell people all the time is, 'Let's try it, if we fail we fail together.'” Alicia also identifies the importance of trust when evaluating teachers. She recognizes that because of the trust she has built within her school, she is able to move forward with the evaluations. "My staff has thanked me a lot. No one has voluntarily left in the last five years since the evaluation came in, and our school scores have just continued to grow so, we must be figuring something out right."

\section{Relationships}

Building culture is a lot of work, but the administrator identified the time spent working on building trusting relationships as essential.

Margaret states she spends a lot of time working culture at her school. "It's a ton of work, culture is a ton of work." But she emphasizes that focusing on culture is important work to do. "My big thing this year is culture. It's always my big thing. Everyone thinks I'm ridiculous, but you know, I really feel that relationships are everything. So now because culture is finally okay to put as a big overarching thing, that's my big overarching thing." Margaret recognizes that working on relationships and building the culture allows her to have a positive school climate. "That is one of the best things about this school is that everybody comes to work, to work to have students be successful." Because everyone at the school is focused on helping students succeed. Margaret identifies, "I feel like its just good energy."

Becky also emphasizes the importance of relationship building within her school. She notes that relationships help her develop a positive climate and culture at her school. 
"What I think we have going for us is a pretty good culture where they do take care of each other."

Alicia identifies the importance of building relationships to create a positive culture within her school. Part of that positive culture is the importance of respect. "If you don't have that respect throughout the whole school, you'll know it." Because of the positive culture, her teachers are willing to work together on their own time, "So, I would say that's a pretty significant amount of time that they are allowed to work together, they don't choose to work together on all of those times. However, they do it more than once a week, they do it more than twice a week."

Zachary identifies the importance of developing a strong climate and culture in his building. "I don't think that happens that easily if you don't have the relationships already in your building, if you don't have the knowledge of your building, if you don't know how to do things so you're not taxing that many people. You have to make sure your best people you don't burn out." Because he takes the time to build relationships within his school, Zachary knows his building well. "You've got to know your building. You have to know when to call a meeting. You have to know when to address something and when not to. You have to get the key people involved and you have to keep them involved. You have to keep them engaged." He also knows "that can be tough to do when there's some, there's a lot of hostility." Knowing your building and your teachers is not an easy task. "Really, in a building this size, it took five years to figure out the culture, and another three to change it." He says now that he knows his building so well he "can tell you, before we do something, 99\% success rate or not. Cause I know the building so well." 


\section{Recognition}

Margaret identifies that another part of relationship building is taking the time to recognize important events that happen in the school or for people who work there. "So that when somebody has a baby, becomes a grandma or whatever, my secretary takes the time, culturally, to make sure we have a luncheon, and all the clerks come over here." She wants to ensure that everyone feels recognized, important, and part of the school community. "It is very important that the secretaries and clerks are recognized and that they feel like it's their school just as much as it's my school." She also celebrates events that happen in the lives of her teachers during meetings.

Another way Margaret recognizes her teachers is through a "pay it forward type thing," where staff recognize each other for doing something good. "The Assistant Principal started the first one and she gave [a bag with a ribbon it in] to somebody because she noticed they were doing something [good]. Then she had to write about it. I take what they write, they email me, and I put it on my blog that Friday. I take their picture and it has nothing to do with me, or any of us [administrators]. It's all colleague to colleague and it is such a hit!" There were twenty-one ribbons in the bag and once you get recognized, then it is your turn to find someone else to recognize. "I'm only doing it second semester because if you do it the whole year everybody gets a ribbon and what the hell is that worth, right so...half a year. You would be surprised [who they recognize] on another floor, around the corner. The custodian got it from the maintenance department chair. Just lovely, lovely gestures. And that was really huge this year."

Garry also takes the time to host celebrations for various things that happen at the school and recognize staff. He had one teacher who went out of her way to help a new 
teacher. "Then of course I would do things for her to make her feel good about it, and just to try to build up and recognize that you are giving up a lot of time to meet with her. But they like each other. They could meet like this at the coffee shop. I remember one question was, 'Do we have to meet at school?' No. I don't care. There's Starbucks down the road. And they were like, 'Oh, Let's go.' And they really ran with it.” He tries to find ways to support his teachers and recognize what they do for him and for each other throughout the school.

Something Zachary does to help with the culture of the school is "We take our content leaders out for dinner as our last content meeting. We feed them. We appreciate them. And then we don't do it [recognize staff enough]. We don't say thank you enough. We don't do everything we should. We don't have time. We should make more time but I think the culture's important."

$\boldsymbol{R Q}$ 3: How does the RI educator evaluation system assist administrators in supporting faculty professional growth?

When discussing the ways in which the educator evaluation has helped them support their teacher's professional growth, administrators noted that the evaluation system itself had some positive elements that they have used to establish and communicate clear expectations of what good teaching is. The administrators used those elements as a framework for providing professional development and support for teachers. When delivering feedback on the various elements, administrators would cycle back to the PD provided to engage in conversations about good teaching practices. When they noticed something happening in the classes, they would use this information to 
inform decisions about future PD activities for the whole faculty or target specific individuals for additional support.

\section{Framework, Expectations, and Support}

Initially, all of the administrators provided PD about the components of the evaluation system, and specifically the observation rubric. This created a set of common expectations and developed common language for teacher and administrators to use.

Margaret used PD time to support her teachers' understanding of the observation rubric. "That's our PD of the beginning of the year too. How you are going to go from a 3 to a 4 [on the rubric]? This is what we saw this year and this is what we have learned from these particular mistakes." The strength of the rubric language is also helpful for new administrators. "My assistant principal was brand new so she relied on the rubric, so it took her a lot longer, but she really relied on the rubric and she would highlight things for people and she would say, 'I'm between a 2 and a 3 here you know."' Her assistant principal "likes it because it's very rigid and she was able to use that rubric to help her in her first year. So as a first year principal it really keeps you on the straight and narrow."

When Alicia began evaluating teachers she looked at the 4-point observation rubrics and "started at a place where really everybody was a 3." She said she also "had people who were already doing $4 \mathrm{~s}$," but as they explored the evaluation system and the rubrics as a faculty, "we talked about that, we showed some examples. That whole year we were talking about best of the best lessons." When looking through the lens that her teachers are already doing a good job, her focus shifted. "So on that regard I come at it from, they are doing a really great job, so how can I coach this to give some feedback to continue to improve?" 
Becky finds that "the content of the evaluation is good. It really does hit most of the best practices." She also identifies that the evaluation system has "helped with the common language. I've said that for a little while that this is what good teaching looks like. I don't care if you are talking math, science, English, social studies." It allows her administrative team and her teachers to have conversations about the "whole idea of good teaching is good teaching," as well as looking at "best practice." By using the evaluation system, she "was able to bring that conversation back to some of that best practice stuff that transcends no matter what department you are in. So I think it's helped in that way."

What Becky sees as next steps is, "do a little more looking at some of the language around what does it look like here." By focusing on her school specifically she says, "We're not talking in general. We're talking in our school this is what it looks like." She feels like "the bones of the evaluation system, of what's in that rubric, are pretty good." Using the rubric "helps open people's eyes." She uses the rubrics to help set common expectations among all staff and create a common language they can use throughout the school.

Garry recognizes that the current evaluation system has set high standards. "The evaluation system is much stronger than what we had at the district level." Some of the positives of the evaluation system that Garry notices are the conversations that he can have based on the rubric. "We at least have nice, clear criteria, and we know how to achieve it."

Zachary identified that the evaluation tool helped set clear expectations for teachers. "You know I think a lot of times they see what they're doing as being above and beyond, and sometimes they don't realize that our expectations have changed." 
Expectations now are different than what they used to be and the rubrics clearly outline what is expected in the classroom. "And what we expect teachers to do now is grossly different than even ten years ago."

\section{Feedback and Conversations}

Administrators use the language of the rubrics to facilitate conversations about good teaching, justify scores, and encourage teacher reflection and self-assessment.

Margaret always attempts to get her feedback related to observations to teachers as soon as she can so they know what they need to improve. "I try not to leave their room until I have sent them their stuff. They get it within twenty-four to forty-eight hours, so that they can try to make it better for the next time." Margaret's entire administrative team works on supporting teachers through their feedback and supports. They are all about "supporting you, you might feel like you have a big shiny light, but I'm not trying to get you, I'm trying to make you better so I that don't have to do anything else. I'm trying to get you prepared." They also work on setting clear expectations about what they do and don't want to see in the classrooms. "I need that to not be that in the classroom when I walk in there." They work with the teachers not against them "I need to know that you're not fighting me all year long."

Margaret and her administrative team use the observation rubric as a way of discussing observations and supporting decisions made regarding ratings. "What you can do is you can pull out stuff from the rubric and show them that there is much more of this happening in your classroom. Just because you use a Popsicle stick doesn't mean you are a 4 in that area." She uses the rubric to help clarify scores with teachers. "If you tell me 'I have used Popsicle sticks,' I will tell you to go pound tar, but if you literally have done 
all these things and I just didn't see some stuff, I will come back in." She is willing to discuss teacher scores she assigns during an observation, but relies on the language of the rubric to support her decisions. "I will go back in and look at it, but I will also say, 'See the rubric, I'm seeing a lot of this and I'm only seeing a little bit of this stuff." She always strives to get it right the first time but is willing to have conversations with teachers about scores. She will tell her teachers, "Thank you for advocating, that's great. I'm not above being wrong." This flexibility and conversation have helped to make the evaluation a positive experience for everyone involved.

Alicia spends a lot of time with her teachers discussing the rubrics and focusing on ways to support her teachers' growth. Utilizing the observation rubric, she notices, "all the $3 \mathrm{~s}$ are what teachers are doing, all the $4 \mathrm{~s}$ are what are the kids doing." She also states, “we talked about how a 4 doesn't just happen in those areas without a lot of work."

Becky feels like focusing PD around the evaluation system and setting clear guidelines around what good teaching looks like at her school gives her "something to go back to. I am able to say things like, "having a clear posted "Do now, target, and closure," would have helped you in this particular area.' Or 'Consider using your closure, consider talking about the closure at the beginning of your lesson.' So I'm able to at least have something to go back to when I'm giving that feedback and that helps, a lot." She sees value in using the school expectations "as a basis and then go to those next steps. At least it gives us that common language, and allows for that peer evaluation and self evaluation."

Becky also has conversations with her teachers clearly defining expectations. "I say, 'I didn't see this at all. Next time I come in I would like to see it,' or, 'can we have a 
conversation about assessment because I'm having a hard time finding enough evidence.' I'll tell them that, and then we will have a conversation. That's when I've had the best conversations, when I put comments to the side like 'Assessment? Is there something I'm not seeing?' Then all of a sudden people will email me, 'Can we meet? There was a preassessment and here's what I did.' When I put something in a comment that is not so favorable, they'll come and we'll talk at that point." By using the specific rubric language she is better able to clarify expectations for what should be happening in the classroom and have conversations with her teachers about how to improve practice.

Thomas also tries to use the evaluation tool to have conversations with his teachers. He meets with his teachers and says, "I want to focus on the two strengths you have and let's make them even better." He also meets with them stating that it, "make[s] sense to have this common data point based on where students are and where we want them to be and have those conversations about growth, and to concentrate on those practices that will get us to that point." He recognizes that the data provided by the evaluation helps in these conversations. "I think there has been some success, you can see we are still trying to focus on data, but to start to focus on where students are as far as baseline and sort of have an ongoing look at what they are doing." He used the data from observations as well as SLO data to help guide teachers' practice.

With expectations clearly defined, administrators were able to see the change in practice from some of their teachers. They identified that teachers wanted to do well and were committed to making changes in their practice. They saw increased accountability as well "a-ha" moments when teachers were able to identify what they could do 
differently. The data they collected also helped the administrators to reflect on their teachers' skills.

Even though there are limitations to the rubric, Margaret sees it as a way for teachers to think more about engaging students in the learning process. "That rubric, although it is not perfect, has really made teachers have these engaging conversations with students. It really has been student led because to get that 4 , I tell the teachers, a 3 is great, a 3 is great, they don't care, they want that 4 . And so if you want the 4, this is how you get to the 4."

Becky encourages her teachers to self-assess and evaluate themselves on the rubric, then she will meet with them and discuss. She encourages teachers, "'Evaluate yourself, what numbers do you think, before I give you scores. Let's go through it together. What do you think you would have gotten in those areas?' Those conversations have been the best. Because then we have that, 'Well, I have a 2 and you have a 3 . Why? Talk to me about that.' That's been really good." She uses the evaluation to help support her teachers' reflection about their own practice and feels like she has "a small group that's excited about having grown."

Thomas identifies the rubric as a positive resource and uses it to define good teaching and encourages his teachers to work towards those practices. "You hope that the rubric, too, is pushing people towards a vision, toward more student-centered learning." While he sees limitations from the evaluation system, he also feels, "we have done a very good job, I think, at moving the people that really make the difference, they have grown a lot." Thomas uses the positive culture of his school to have difficult conversations with his teachers. He tries to use statements supported by data such as: "This data we are 
giving you here, it isn't about blame. The data in your department shows you spent $80 \%$ on lower order questioning and discussion in your class. Okay, you say you want to be at $60 \%$, how do we move the needle on that and let's have a conversation and let you guys have that conversation. Let's try something and let's look at it again."

Garry notices that with the clearly defined rubrics, "people are looking at that a little bit more on their own." He notes that teachers are beginning to review and revamp their familiar lessons more now. "Sometimes as a veteran, too, you kind of fall into a pattern, and this assignment is great and I do it every year. It's awesome and people love it. The kids love it. The parents love it. Yeah, but then how can you redefine it a little bit? Especially getting standards in there maybe, and then it makes them think about the rubric and that's really what it comes down to. And then you have things you didn't even think you were doing in there." The evaluation rubric has made people look at the rigor of their lessons. "So maybe rethink that assignment a little bit."

Garry also commented on the additional accountability provided by the evaluation. When he is in classes now he recognizes, "They know I'm around more but it's not just me popping in, it could be some other reason and when I say, 'hey I noticed about your questioning...' when I say things like that now, it's like 'ding, ding' a bell might go off, 'that might go on my evaluation now."” This has helped with teachers holding themselves more accountable.

Garry also finds that his teachers "are dedicated, and they really want to do well, which I think is one really big plus." He finds that "people overall are buying in." And he sees that "people are used to the evaluation model, I think they are a little bit more open to some input and feedback." They want to see improvements. He also comments "no one 
got 1s and 2s, like it's not punitive really." His teachers "want to grow" and he is able to use the rubric and the evaluation system in general to help with that.

Zachary states he has been able to use the rubric to have some reflective moments with his teachers. "I will tell you I've had some really good moments with teachers around the evaluation system, like "a-ha" moments. Very positive where they've seen something, or we've both learned from it, or you know, where we both realized that there's a weakness then how do you build on it. It's really nice when a teacher will say, 'I now understand that this is a weakness. I now look at this rubric. How do I get better? What do I do?"' But he also recognizes that teachers are not always as reflective on their practice as they could be. "I think sometimes their reflection is not as strong as they can be. Or sometimes I wonder if we've taught them to reflect at the level that we need them to. I guess it's hard to look at yourself and say you're average in certain areas."

Zachary also states that although he's not sure if "teachers are looking at the evaluation and reflecting on it the way you would want them to," it provides him with an opportunity to identify strengths and weaknesses with them and review data. "The evaluation has been good at pointing out some of our weaknesses, some of our strengths. And data. The data collection helps with that. I think it's also made teachers dig a little bit deeper in their profession." The evaluation tool has also given him some language to help teachers who want to do their best. "Teachers in general are such perfectionists and they always want to do well and they always want to be the best of the best."

Zachary has seen some positives from the evaluation system around using data. "I've seen some "a-ha" moments with SLOs." He has seen some teachers "looking at their data saying, 'I cannot believe they aren't doing well.' Yes, very positive around data 
use." He identifies the new evaluation system, "made our teachers not only do a dog and pony show with data but also understand it better, and not only understanding it better, they can take action and reflect on it." So for some of his teachers, he believes that "it has been a big eye opener."

Zachary identifies that he has also been able to reflect on his teachers' skills. "Every once in a while I think I have a strong teacher in an area and through the evaluation system we have found they're weaker than we thought they were. And that's been good. Because they're still strong teachers but in an area where I might have wanted them to do PD, I don't because I'm starting to say, 'wait a minute, that teacher's not as good at problem solving as we thought they were.' But you know, sometimes you have rosy glasses too. They are the best teachers and you don't realize where their defects are."

RQ 4: How does the RI educator evaluation system hinder or challenge administrators in supporting faculty professional growth?

Administrators in this study were faced with several challenges with the implementation of the evaluation system and trying to provide support for their teachers. Many administrators felt that the evaluation system itself did not provide the level of accountability that they felt was intended. They recognized that some teachers seemed focused solely on scores and not on growth. They faced pushback from those who were resistant to change. Other restriction they felt were the limitations of time and paperwork involved in completing the evaluations, their ever-increasing roles, and all of the additional mandates and initiative they have to focus on in addition to the evaluation. 


\section{Accountability - No Teeth}

Lack of accountability was a major area of concern for the administrators. An early concession that was made to the RIDE evaluation system was that teachers were put on an evaluation cycle depending on their evaluation rating the previous year. Alicia does not like the change in the evaluation system that makes it cyclical for teachers. She notices a lack of accountability for teachers the year(s) they are not being evaluated. "I have to say that I think the worst part of the evaluation system is the fact that, that they did it like this, that they made it cyclical. I thought the year that we all had to do one, and everybody had two academic goals and one professional goal and one classroom evaluation, I thought that was perfect."

Margaret also finds that when teachers are not being evaluated they "don't have to prove anything so the accountability went backwards. So that was a bummer for the principals, I think, because I had everybody looking at the data, now I have just the people who are on."

Garry agrees that the cyclical nature of the evaluation takes away accountability. "The other people who were not evaluated didn't have to do anything." Since so few people were on the evaluation cycle teachers were missing the teamwork and togetherness. "I think people were like, 'Oh, I'm completely by myself,' and they were used to being more like, think-tank, let's really go through this. 'Oh, I like your goal, because I might need some of that,' or they could tweak somebody's goal based on what they were doing."

Another change that was made to the evaluation system was made to the improvement plan. Margaret feels that the current structure of the improvement plan is 
not robust enough. She states, "Well I'm pissed because he [teacher not performing well on evaluation] needs more than the improvement plan. I think I'm going to have him do like two improvement plans."

Becky also sees an overall lack of accountability on the part of the teachers and does not find that the evaluation itself holds teachers accountable. "The evaluation is not helping with the accountability piece. We recognize the importance of accountability, the evaluation is not helping us in that way." Becky recognizes, "They care once they get their scores. Does it change their practice, no? They'll complain once they get their scores." Despite the complaints there is not always change to classroom practice. She states, "They aren't nervous that they are going to get a 2 on their evaluation. It's not the evaluation. The accountability needs to come from peers, from inside."

Garry identifies that teacher ownership of the evaluation is also a problem. "I still feel like teachers need to own it a little bit more. Because I'm showing them the documents. I'm going through their handbook. They still haven't embraced it at all. It's like, 'I'm going to do what you need me to do. I'm going to do what you tell me to do.' But then come time for pre-conferences or those initial conferences this year, no one had even looked at their handbook. 'What do you mean the forms changed?'” He tries to keep his teachers informed, but they don't own it and 'they don't read the evaluation documents until they need to, but then it is stressful for them because they don't really know."

Thomas also identifies lack of accountability as a limitation of the evaluation system. "We are trying to drive accountability, but you can only drive that with a percentage of your faculty." Most people "just give us that freaking data at the end." He 
also said, "I asked some department leaders not too long ago, what percentage of people are really zoned in saying, 'I've got to get better outcomes and I've really got to work on these couple of areas to try to really be intentional about my practice so these outcomes are better.' I mean, $25 \%$, definitely under $50 \%$."

Thomas identifies, "personally I don't always see the bang for the buck with the formal evaluation. I think a lot of it is still a dog-and-pony show." He struggles with the evaluation in the sense of assigning scores. "I think it is very difficult, I mean you can be honest with it and give people $2 \mathrm{~s}$ and $1 \mathrm{~s}$ and this, that, and the other thing, but once people see scores it's like this punch in the gut and it doesn't do much for them." Even when identifying less-than-effective teachers, there are limitations, "Are you going to get rid of them in five years?"

Margaret also struggles with the limitations and challenges of terminating a staff member with the new evaluation system. About one teacher who is in need of improvement she states, "Not that he wants to change anything. He wants to agree to disagree, and I'm not okay with putting twenty-eight kids in his classroom if he's not changing. Now it's hard to get rid of somebody." The system does not have the impact that administrators are looking for in that way.

Zachary also identifies that, "it's very hard to terminate under the new evaluation system." And while he recognizes, "maybe evaluations shouldn't be about termination, but at some point, you have to bring a name to school committee to terminate, and it's very hard to do when their attorney or their union says they've been effective the last three evaluation cycles. We don't terminate effective teachers. Right. But they are always developing or lower on their observations but they're effective [on their overall 
evaluation] because of their SLOs." Zachary sees this contradiction between observation scores and SLO scores as one of the ways in which teachers manipulate data, which I will discuss next.

\section{Pushback and Manipulation}

Because teachers identify their Student Learning Objectives (SLOs), teachers can create one they are sure students will meet. In this way, administrators feel that these data are easily manipulated. Garry recognizes that there was some inflation of scores. "I felt like after the first couple of years people were getting higher scores because their student learning objectives were coming out really, really high, and I felt like, 'I would have given them a 3 for their final rating,' because that's just normally, just my gut, and they got a 4 and our superintendent was like, 'Well, what are you going to do? This is it, this is how it shakes out." He believes that final effectiveness ratings for some teachers were inflated. "We had a lot of 4, and they are not 4s."

Becky identifies one of the "challenges behind the evaluation. I just I hate, I hate SLOs." When the focus is on the outcome and the numbers, Becky has seen manipulation of data. In relation to the badging system that she created she states, "You don't know if it's really moving people. What happened at one of the PD days was, 'OK, you can get a badge if you get an $85 \%$ or better on this particular assessment.' They were cheating! They were cheating! The teachers were cheating."

Zachary also recognizes the challenges of SLOs. "SLOs are tricky. We go back and forth whether or not they are rigorous enough, what is considered rigorous enough." He finds that with the high-stakes nature of the evaluation, especially in his district where the evaluation rating counts as part of seniority for retention, promotion and tenure 
purposes, teachers are focusing on the number they need to get rather than the process. "People are just looking at their numbers, cause that's what they need to be highly effective. 'Did I meet the number? Did I make it? I need to get two more points here.' It's a points game." He notices that teachers are well aware of the numbers before he goes in for an observation. "I don't do the math in advance but they obviously do. They are constantly saying, 'I need to make sure this, I need to be effective here.' Or 'I need two more points here.' So it's not about the teaching and learning." This focus and pressure on numbers and final effectiveness ratings, "it really impacts your ability to evaluate them."

Margaret identifies some pushback comes when teachers receive scores from observations. "I've had people challenge me on the scores." The pushback is "really between the 2 and the 3." She states, "When someone gets a 3 instead of a 4, I don't really hear a lot unless they really think they should have had it. But between a 2 and a 3 , I hear it. I hear it."

Becky also recognizes the teachers' focus on scores. She tries different strategies to shift the conversations so they are focused on the information presented in the rubric not the score. During the observation "I send them the script and I send them feedback as I'm thinking. So I'm scripting and off to the side I'm putting my little bubbles and my comments and I send it to them and I say, 'These are the notes that I took during your evaluation. Please review, if there are any changes or things you want to see made, please let me know prior to my posting the scores.' Because I didn't put any scores, so I let them kind of stew on it for a week or so, and then go in and put the scores." 
Garry also identifies the negative reaction of teachers to the evaluation. "People felt like a little more threatened the first year, you know, when you give them some advice or some feedback and you get a little bit like, 'oh, I'm insulting you."' One specific, challenging situation he relayed was, "I had one teacher two years ago who did not do all that well. It wasn't pushback. It was more surprise, more like, 'Really? I can't believe you rated me this way?' It was a lot of coaxing and talking and feeling it through, but it was only really one person."

\section{Negativity about Evaluation}

Another area of concern administrators identify is the issue of negative feelings that teachers have about the high stakes nature of the evaluation. Margaret identifies that she has some staff that are resistant to change and they want to do things "the way they've always done it. I think what they need to understand is yes, they are doing a great job, but if they add this piece their great teaching will be even better." Margaret recognizes those negative feeling and tries to offer support. "And when a teacher is feeling scared like that, that's when they talk and I say, 'Come see me. Really, I really do meet with people. I really do talk to you. I talk to you in the hall or the class."”

Alicia notes that if a teacher does not do well on part of the evaluation they feel embarrassed. "Teachers are always ashamed. I don't know why nobody can get help. It's always shameful." With a high-stakes evaluation, "they feel like it's their job on the line and they are feeling scared." She also identifies a lot of pressure to do things right, "I think unfortunately a lot of the additional things, the stress that principals were under to make sure it was done correctly, put a lot of stress right back onto their teaching staff." 
Teachers were apprehensive about the evaluation. "I would say, that they were more resistant of the system than of me.”

Thomas tries to shift the focus of the evaluation from being punitive toward a more supportive and growth opportunity. "I think we are still dealing with some of the residual effects of 'You lose your job, you lose your certification for life' being the main focus." Thomas notices that his teachers focus on the scores, "That's all they care about." He tries doing things differently with the evaluation. "I did a thing where I didn't give them scores at all until the end to see if we could just concentrate on the feedback." He also tried to "do it all verbally, because I think tone is everything."

Garry did get some pushback from teachers for "anything new, anything that was either labeled RIDE at one point or labeled new." He recognizes that, to some teachers, "it was more of RIDE was a bad word kind of thing." This distrust of RIDE, district administration, and school committees was echoed by others as well. Margaret feels that some of the negative attitudes that she is dealing with are left over from previous administration or resistance that the teachers feel toward central office administration. "I've been trying to say that for the last two years, but teachers do not believe me because they do not trust administration or the school committee." Some teachers have had issues in the past “from so long ago but they couldn't get over it, so we had to work with them literally for two years, meetings, meetings, meetings." And as hard as she works, "there still is distrust. I can do what I can do." She continues to work on it stating, "And they might be untrustworthy of the administration as a whole but I'm working really hard because when they say, 'they, but not you,' no. I'm them; I need to change that culture. 
So if we are going to have a better relationship here, I don't want people to fear me like I'm going to come down like a hammer."

Thomas also recognizes that there are some issues with trust of administration and central office administration from the past. "I think this district has very little trust for central office or high school leadership at the time, so we've been trying to develop a lot of that." He also states that sometimes decisions made at central administration do not reflect what the local administrative team would do, but it is hard to separate from that. "You know our central office might act in a way we wouldn't sometimes. We are caught up in it. They operate in a way we wouldn't sometimes with people, and so that sort of hurts trust a little bit. Because the teachers obviously think we're in those conversations and wrapped up in that kind of stuff, but not always." He also recognizes that the evaluation undermines the trust they are working on developing, "all that trust goes down the toilet as soon as you give them that score."

While there are some teachers who have responded positively to the increased PD and supports related to the evaluation system, Becky recognizes, "you still had those same people sitting in the back that aren't listening and aren't doing." She still struggles with the idea of "tapping into the unmotivated [teachers]. It's like any classroom, am I tapping into the unmotivated [learner], nope. But you have eight hours of your own personalized learning time and if you're choosing not to use it to your benefit. I can't make you."

\section{Time, Paperwork, Limitations}

Zachary does not see the benefit in the evaluation system for the time that it takes to complete it. "The evaluation system is a bear that is not producing the results that you 
would want. That's what kills me. If I walked in the classroom and saw improvement in teaching and learning based on the evaluation system, I would be thrilled. I would say it's all worth it. I don't see that."

Zachary notes, "I do think that EPSS [the online evaluation documentation program] is not the end of the world, now that I know how to navigate through it and I know how to beat the system at it's own games." Within the evaluation documents he does "a lot of cut and pasting, there's a lot of 'see mid-year." He believes, "the forms are redundant. I believe there's too many." One example he gives is "end of year, I have to go in [to the system] and do every teacher that wasn't evaluated that year, and I have to go in and check off 'not evaluated.' Now that doesn't sound like a big deal, but when you're doing it for seventy teachers. I timed it. It took about six hours, from start to finish to do it over the course of a couple of days."

Another issue Zachary identifies is the actual time required to complete the evaluation process. "It's extremely time consuming." After conducting the evaluation, "anyone that is in question of not doing well, none of that's going on EPSS until I write it." If his assistant principal "has a tough evaluation, many times he will send it to me on a Sunday and he'll send me a text and say, 'Hey can you read it.' And that's not a fifteenminute read. It takes an hour to read something like that, then an hour to respond. And takes him an hour to figure out what to do with my response. Then he has to respond back to me." The paperwork needed to compete all aspects of the evaluation is very time consuming.

Zachary and his assistant principal "spend a lot of time at the beginning of the year" approving SLOs. They "approve all SLOs together," no matter who evaluates the 
teacher. They "probably set aside four hours a day for a week to approve SLOs -- just to approve them, and then when we don't approve them it's emails. It's back and forth; it involves both of us, like detailed. Same thing happens with mid years. We meet on every one for professional responsibilities, so that we're in agreement. We sat at this table and did it together. And we shut the door and it takes hours." Observations are also time consuming. "We made a commitment to our people. We don't stay for twenty to twentyfive minutes. We stay from start to finish. What happens when we don't, teacher says, 'you nailed me on, on assessments. But I gave an exit question, a great exit question.' And then they show it to you and you're like, 'Oh my God he's right.' So we stay from start to finish." In addition to doing the observation there is also the documentation for completing the observation in EPSS. "Ideally, if you do it right, you do an observation and you come back, and that day you write it, it's all fresh in your head. What about when you do three in a day, and you have that irate parent that comes in demanding to see you?"

Margaret also struggles with trying to balance the new mandates and initiatives with current expectations. She recognizes that, "of course it's layered over top of all the other crap. It's not like I'm taking anything away." She also tries to recognize that, "people are going to shutdown if you want them to do too much." Given all the additional mandates and initiatives, there is not always a lot of time to focus on everything. Margaret identifies that while some of the initiatives are good and may already be happening in her school, documenting them also takes time. "But then to take the time to formalize it, takes a lot of time. So then you get your eye off of student success so that could be a struggle." 
Garry has noticed that his role as an administrator continues to grow. "It gets a little frustrating just because I'm responsible for the day-to-day building things, but I'm also the LEA for all special education meetings and I do all the RTI [Response to Intervention] meetings." That has become a larger part of his job. "We had thirty kids that had RTI plans last year. I had thirty-five IEP students so it's huge, I mean two days a week I'm in meetings." He states that his role has shifted to become more managerial. "Maybe three years ago now, I felt like I've had to do a lot more managerial duties. I felt like more of an instructional leader in my first seven years, more of a manger in my last three. It's just this shift. I've just felt like I've done a lot more management rather than leadership. Where I feel like now is the time for more leadership. And we just met about that this week as an administrative group, and we've got to get back into it. It's funny, all of us are feeling that way." He notices a shift even more so once the evaluation started. "I just felt like I was spending more time on paperwork. So with one came the other." Garry references the issue of not having enough time to get things done. That includes the additional expectations added because of the evaluation system.

Zachary identifies the challenges of balancing managerial roles and supportive roles while also recognizing the importance of both. "I don't care how your teaching and learning is. If your building is dirty and your toilets don't flush, kids don't feel respected. They don't want to work. It's not a good learning environment." Zachary states much of his time is spent on managerial duties. He identifies that he can't spend all of his "time doing evaluations or this building wouldn't run." He recognizes that "to run a building is time consuming. I know you know the philosophy that we're educational leaders, but we're still managers too." Many of the tasks that he attends to daily have "nothing to do 
with teaching and learning. It has nothing to do with the educational leadership of the building."

\section{Restrictions of the Evaluation}

Zachary feels the current system is not as efficient or effective as past systems. He discusses that, for some districts the evaluation has "been a godsend." But in Zachary's district they have "been evaluating teachers beyond what the state has required for twenty years probably. Or has had a system in place where no teacher goes thee years ever without an evaluation. With checks and balances in place. We don't hire content leaders unless they're highly effective. We've used our evaluation system the twelve years I've been here, for not only accountability but to make sure our best people are in the best spots."

Zachary also feels the observation requirements of the system are restrictive. "I don't believe every teacher needs three observations. I have some teachers I walk in they're outstanding. And you're like, 'I really need to observe this person?' I went in unannounced and it's all 4s, why do I need to go in two more times?" By knowing his building, he is able to know which teachers need support and which ones don't. "When

you walk in unannounced and you have a teacher nail everything, you start talking to kids and they answer everything, and you walk in for a five minute walk-through three more times and you see the same thing, and you know every kid, every parent in the sixth grade wants their kid to have this teacher. Every teacher wants to work with this teacher. Every time you ask, they step up. Why do I need to do all that? Why do I need them to show me their professional foundations?" There is no differentiation for teachers at 
different places, "the evaluation system has set it up that we have to look at everyone the same."

Zachary sees the current system as restricting his vision of a productive system. "I will also tell you before the new evaluation system was in place, I visited every classroom every day. I don't do that any more. I have fifty classrooms. I used to get into every classroom every day. Can't do it any more. Because of the paperwork." If Zachary could design his own system, it would look different. "I don’t think we would spend any less time evaluating people, it would just be more productive and it would be where it is needed. Where now I'm just spread thin...I don't think we have the bang for the buck... You're not getting your return on what you put in. I don't think it's worth it...there are so many places that we could be spending our energy that would improve teaching and learning, or impact kids lives."

Thomas and his administrative team put a lot of time into doing evaluations and they try to make the evaluation work. But he emphasizes, "That's when I say bang for the buck. I don't think it's in this evaluation." He also states "imagine if we put that time into that other system that I was talking about where we have that vibrant culture of teachers looking at themselves on video and having those conversations and we got into those groups as teachers, small groups of teachers watched a video and had conversations."

\section{Balancing Support and Evaluation}

Administrators identified positive and negative aspects of the evaluation system, as well as discussed how they provided professional development to support teacher growth. When asked about balancing the two, administrators discussed working to reduce 
stress associated with evaluations, developing other methods of accountability, remembering to use relationships and to consider the human side.

\section{Reducing Stress Associated with Evaluation}

With the implementation of the evaluation system came the introduction of many new evaluation elements. The administrators identified ways that they tried to use systems that were already in place to help their teachers with data collection for the evaluation.

Margaret spends time during professional development helping teachers navigate the online evaluation tool EPSS. "One of the supports that I have to do is I have to show everybody EPSS again." In addition to supports on EPSS from the administrators, "all department chairs were expected to know how to use EPSS." Because of some of "these little nuanced changes in the rubrics, people didn't realize what was expected from the last time they did it." She also offered supports "in our computer labs, people who are doing their artifacts. I had people in there just to help teachers upload materials, so they would know what they are doing."

Thomas said they try to simplify the process for teachers as much as they can. "We usually do a group beginning of the year conference. We set for the school a professional growth goal that has been common. As much as it's common, it's personalized in the sense of those eight hours they can answer however they like." They try to make the evaluation process part of the PD and supports they offer their teachers.

One of the things that Alicia tries to do to reduce the stress of the evaluation is to make the paperwork part of the evaluation easier. "Instead of people putting together binders of what they learned, we shared out at faculty meetings what people learned." 
This also helps because, "people all worked together and then we did share-outs. So that just brought our whole school forward about what other people were learning and doing." Alicia also states, "I never asked for a lesson plan." Many teachers found the lesson plan writing stressful. "A lot of principals were asking teachers to write lesson plans. And I looked at my staff and I said, 'I would hope that you aren't writing a lesson plan every night. If this is what you were producing every time you taught a lesson...no."”

Alicia tries to use systems they already have established at the school as evidence of documentation for the components of the evaluation. "I remember the first year, two years, people had to give in binders around the district. And then I was asked how come my teachers aren't doing a binder. Well, I explained our thirty-day plan and in my mind, this is what I get out of this thirty-day plan documentation." She limits the documentation her teachers have to turn in. "I also laughed at people who were printing out emails upon emails upon emails. I was like, if you print all that, I have to read all that, that's awful. Haven't you already taken care of that? I can tell you when you haven't taken care of that, because the very next email comes to my email box, I got that. So I don't want to. But if there is something you want to show me because you are particularly proud of that, then feel free to show me. I want to see that. But please, don't wait until May to give me that. Give me that when you get it." Because of the ways she tries to support teachers and use systems that were already built into the school as documentation, she feels that teachers were less stressed about the evaluation. "In our school it wasn't as stressful, I don't believe, as it was in many other schools."

Alicia identifies her time as a support that she provided her teachers when the evaluation first began. "I would say that I also give my teachers resources of time in the 
sense that when we were doing the evaluation for the first time, it wasn't a gotcha system, if you didn't remember how to upload it, come see me and we will upload it. It's not a gotcha kind of thing. Yeah, they definitely get that." She would meet with her teachers as often as they needed to support them with the evaluation process.

Garry also provides some flexibility with the amount of paperwork that is collected. This flexibility comes from the superintendent. "You stick to the evaluation but the superintendent is really more of a, 'I know what you do. Don't get caught up in all of this paperwork and all of the other stuff that goes with it, but we're going to stick to it. But let's have an honest conversation. I'm going to tell you where I think you are. We can go back and forth and maybe you can prove otherwise.' And he gives us full say on what we need." Because of this flexibility from the superintendent Garry has, "kind of weaved that into [his] style" as well and not required his teachers to collect a lot of documentation as evidence for the evaluation.

When the evaluation first came out, Garry spent time developing a feeling of togetherness amongst his staff. It was the positive climate of the school that helped take the stress out when the evaluation system came into play. "And we literally that year, every faculty meeting, every curriculum meeting, before school meetings, we spent so much time on the evaluation, just little by little and easing into everything." He focused a lot of his time helping his teachers with the evaluation system.

Zachary and his assistant principal also try to structure the components of the evaluation to make it easier for their teachers. "At our first staff meeting at some point, we do our beginning of the year conference, for teacher evaluations. We do everyone at once unless they are on an improvement plan or unless they are brand new. Then they get 
individual." They always allow teachers to have an individual conference if they prefer, or they can just participate in the group one. When developing the "Professional Growth Goals we tend to do, we try to do one. The assistant principal and I try to put it out to the building. You have an option to do this or do your own. If you chose this, this is the support that is going to be put in place, or here's the PD, that is going to cover that. So if you attend the PD this is the evidence you can use." They also provide "building-wide SLOs. The assistant principal and I do them, so those are ours and then we put them onto the staff and then we ask them, we give them the option to do them or to do their own. The last couple years they have been reluctant to do ours because ours have been set very high. And honestly one was not attainable and staff knew that so they didn't take it."

Alicia works hard at trying to take the stress out of the evaluation for her teachers. "I'm not going to stress you out about evaluation." She tries to always focus evaluation on improving learning for students. "I think that really kind of talking about what do kids need to do; how do we get kids excited about learning; and how to we get kids to ask those questions? So that is really all about improving learning." She also identifies that being upfront and honest with the teachers helps to relieve some of their stress as well. "I think the teachers had a lot less stress. They can enter into it as a much more willing participant in that conversation because they know I'm not trying to write them up, I'm not trying to get them. I'm just trying to have a conversation about what do I want moving forward." Alicia works hard to create an environment in which stress related to the evaluation was removed and teachers were working together. "So they know, I think, when you do things like that, that takes a whole bunch of 'nobody's perfect, everybody's 
in this to just be a learner and do what's best for the kids.' And definitely know that, they know that everyday I come here I put kids first and I never waiver from that."

One thing Alicia tries to do differently to ease the stress related to the evaluations is differentiates the Professional Growth Goals (PGGs) for teachers. "The district every year comes up with one PGG, and we can select it or not select it, depending on where that meets the needs of our school." Alicia states, "I have not done that at our school. We have not done a whole school PGG at all." When it comes to a whole school PGG, "I don't see that one PGG usually, even if it does, even if one is that generic, how it will take place in different groups will be very different." Instead she states she works with teachers individually and "will suggest different things and different books and then talk to them about what they want for their PGG."

Alicia also approaches the evaluation with the attitude that all teachers have good intentions and spends time recognizing the things that they do well. "In the beginning we talked a lot about evaluation, I said, 'you guys, respect and rapport is just part of our school. Let's not even worry about that." She finds that starting with the assumption that teachers want to do a good job helps her with evaluations. "When you go in through that light teachers can hear that more. Hardly a teacher gets to work and says I really want to do a bad job today. Let me get up so I cannot help kids learn.”

\section{Alternative Approaches to Accountability}

Administrators found that the evaluation system provided limited accountability, so they created alternative approaches to accountability within their schools. Thomas emphasizes trying to balance teacher professional development with personal accountability. One strategy he has used is allowing teachers to tape themselves 
delivering a lesson in the classroom. What he finds is "teachers tape themselves, watch it themselves, don't like it, tape themselves again, say 'I can do better than that,' and tape themselves again. They are actually watching themselves maybe three or four times. Which is good, because they have never done that before." This process helps teachers become more accountable for their own development as they watch and critique videos of their own classroom practice. "I think it ends up being more powerful because the teacher watches themselves four times. I think it's probably more powerful than the dog and pony evaluation."

At Thomas's school they also have an "I-Walk through process, which is a quick, look for frequency of some of the things like questioning techniques or use of technology or room configuration or stuff like that. So we did close to a thousand visits last year." The teachers do the walk through and see each other's classes. This also helps with accountability. "I think there is accountability there that we could never create, from their peers and from everything else."

Becky has created a culture where teachers share out best practices that are happening in their classrooms. "We have done it [teacher's sharing out best practices] at the end of the year for the last two years, but the teachers are saying maybe we should do it two times a year now." She finds this helps with teacher accountability for what is happening in their classrooms. "The teachers share their answer to the essential question, so there's the accountability piece. Because our message is that we care that you are learning the skills as opposed to just coming in and being taught at."

Alicia also created a program where teachers share out their best lessons. It was born out of the evaluation process where she recognized, "I'm learning tons and you guys 
aren't learning anything. I was like 'This is, this is just, this is great for me and I get to see awesome teaching.' And so I floated some ideas out about how we were going to change things and they liked the idea of bringing their best lessons. They wanted to share their best lessons. They didn't really want to share their best lessons, but they really wanted to hear about the best lessons." Teachers asked Alicia to share out some of the best lessons that she saw while observing. "I do have a great memory, so on the spot I went around to every teacher and said something amazing that is happening in their classroom. And they were like 'Ok, so we can do this.' I was like, so you can share what is happening in your classrooms because you all have something to share." She was able to share out with teachers and help them to learn more about what was happening in each other's classes. "We showed some examples that whole year we were talking about best of the best lessons." Then as a faculty they were able to have conversations about their best lessons.

\section{Being Mindful of Humanness}

In the end, administrators need to find a way to balance the supportive culture they are working on creating within their schools with the accountability piece of evaluation. They try many different ways to do this, all the while keeping the relationships and human element of being an administrator at the forefront of their decision-making.

Margaret recognizes that she has some negative staff that are resistant to change. "But the other ones that want it just like it's been always, are very eager to wait for something bad to happen to that really positive person and then they are, 'Oh yeah come, come on over here and let me tell you all the bad things and why you should just close 
your door." She recognizes the negative people in the building, "it's very vocal yet smaller population in the building, yet they are really running the show." But she tries to use the culture in her building to counteract their negativity. The positive people in the school "don't feel like arguing, they're not arguers." She feels that most of her staff are on board and working with her but states, "I want it to be $u$ s and that we are all going the same direction, but there are a few rogue players." She describes push back from an individual teacher "who was like, 'what does this have to do with me?"' In the midst of being frustrated, dealing with negative people, “I don't ever play to the emotions because everybody is watching, so I basically just go back to the data and what the research shows and that this has been helpful."

When teachers are being negative in her school, Margaret uses the trust and supportive culture that she developed to help challenge and address their negativity. She recognizes that there are very negative people who are "just going to do everything they can to bring their classroom, whatever people around them down." But she doesn't let that go, she addresses it, "If some guy is walking around here muttering and muttering, there is a conversation, 'Listen, I get there is some work you have to do, but I would really appreciate if you were not muttering in the hallway, mutter after school, send something on your union email, enough. I can't see you in the hall anymore."” Margaret and Alicia both explicitly state that they would not wait until an evaluation to confront a teacher with concerns they have about teaching. They can use the evaluation tool as a way of starting a conversation, but they want to be transparent about their actions and deal with a situation as it arises. Margaret expresses any concerns she has directly with her teachers. "I called teachers into my office and said, 'I'm not happy 
with what I'm seeing."' Alicia says that she wouldn't use the evaluation system to try to get change in her teachers' classrooms. "I feel like as a principal if I needed immediate change, I wouldn't use the evaluation system to get that change. I would just have that conversation."

Alicia also stresses that she does not use the evaluation as a "gotcha" in the sense that "if I had a problem with something happening in your classroom, I wouldn't be waiting until the evaluation system to tell you about that." She wants to address any issues that she has as soon as she notices them. "My staff knows if I've already had an issue, we've already had the conversation about the issue. I'm not waiting for evaluation time to have that conversation about something I saw that needs to be changed."

Another approach Alicia uses is that she focuses on the positives when she writes up an evaluation. "All the lessons here are strong, but if I want to coach on a point, I often don't write that point down, I just say, 'I have an idea the next time you do writing workshop come see me.' And so that will be kind of a conversation." She prefers to have conversations about areas where she wants to do coaching about a lesson. "Then we will talk about it. I think that becomes daunting when they read about that idea, instead of just having it be, 'I was excited when I saw you do this because it made me think that next time you could do that,' and bring up the whole rigor of that lesson." She finds that writing down positives and talking through improvements "helps ease people and make them more comfortable."

After an observation Alicia had a conversation with a teacher. "I expected something different when I saw a reading classroom. So we had our conversation and you know, it was brought to the level that I expected and that's when I went in to write it up." 
She is also upfront about the way in which she makes her decisions. "I tell people how I make my decision is, kids first, teachers second, parents third." She is consistent in making decisions that way. "And every single decision I make is with that, including when I evaluate a teacher so, really if it works for the kids in that room then there is nothing but positives to say and the only reason I will coach that is if it isn't really working for the kids."

When doing an observation Alicia identifies the purpose of the evaluation. She states when writing evaluations "this isn't a letter of recommendation, if you need a letter of recommendation, I will write you one, this is an evaluation of what's happening in your classroom right now." She emphasizes that the focus of the evaluation tool is for teacher growth and not a letter of recommendation.

While Becky and her administrative team spend time working on culture, they also have to balance that with the evaluation. "What we have to fight against is, "we are not using this to evaluate you, the purpose of us giving you this assessment is so that you can manage your own growth. It's not for me." When giving teachers a pre-assessment or encouraging them to try something new, Becky struggles with teachers seeing her evaluator role separate from her instructional leader role. "That's hard, being an evaluator. 'I don't care that you got only three right on this test. I'm not giving it for my purpose, I was giving it for you.' And that's a different mind-set and that's one way, with that growth and that differentiation, that we are trying to help them at their own level." They have spent time on PD, "really driving down to classroom practice, which when you talk about trust is really, raw for a lot of people because now you're talking about 
what I'm actually doing in my classroom, you're not talking about assessment, now we are talking about where the rubber hits the road."

Garry notes that there have been issues related to the evaluation. "There have been a couple of edgy, more administrator vs. administrator, when we have our administrative council meetings, there have been different styles.” They do not always agree on evaluation approaches. "Some administrators are really hard-core. I don't want to say no-nonsense, because you want to abide by the system, but you kind of lose your humanity of it all, you need to keep the culture high. You can't just knock people down." One instance he recalls was, "I remember somebody said once, 'I walked into her classroom and this, this, this, and it was questioning and...' they just destroyed this person. What is that going to do? I mean have you always had that issue? That person's been in your building for twelve years, so you just walked into that lesson and either you

just noticed it or you're just going to take that snapshot." Garry said, "I remember saying, 'Would you want the superintendent to just walk into your building and you are having that one bad hour of the week and he writes you up for that?"' He felt that "some people, I think kind of took the evaluation the other way, really took the opportunity to back hand a couple of people around. I kind of felt like what goes around comes around. I didn't want to do that at all."

\section{Merging Support and Evaluation}

Through the work done on creating a positive culture a their schools, the administrators try to balance the accountability of the evaluation system with the formal and informal supports they have provided for their staff. This is a daunting challenge. 
Margaret identifies the importance of culture and climate of her school, and understands the responsibilities and limitations of the evaluation system. "I'm trying to marry evaluation and support, because I feel like, culture and climate is the feeling. And I do not accept negativity, I just don't. I talk about positive energy." She continues to try to "merge those two together, and it's because of the time I spent on the culture that I can have those difficult conversations and they know I care."

Thomas is trying to find a way to balance accountability and support. "I've always said that there needs to be an equal amount of accountability and support. You always hear that thing about pressure and support. You need capacity-building and you need accountability structure in place." He recognizes the need for balance, "I believe we have high levels of support, I believe we have somewhat high levels of accountability, but I don't know (laughs) so you have both, then what?" He understands the importance of balancing both and states, "we probably just concentrate more on the support side because that's the side that hasn't been there for so long." He recognizes the importance of a supportive climate/culture, "Imagine what it would be if the support side wasn't there. And we were just doing what we do with evaluations. I know people take a punch in the gut now when they get a bad score, but if the support stuff wasn't there, at least we have a leg to stand on." Without the support "there would just be anarchy, if you gave bad scores and didn't have any of that support. And there are buildings like that. So at least we are sort of trying to give top shelf PD and go from there." Thomas recognizes the challenges of balancing both. "How do we balance? We don't (laughs)."

When trying to address the issue of balancing evaluation and support Garry mentions that "educational leadership is where I want to spend more time, but I feel more 
managerial, it's an interesting dichotomy I guess." With the increased expectations of the evaluation, he spends more time on that. "I would like to spend more time, not necessarily on the accountability piece, but on the teacher leadership piece, the educational leader piece, which kind of dovetails right into the evaluation." He also mentions that balancing evaluation and support is an interesting idea to him "because, maybe I don't see them as different." "I've tried to kind of keep it as one. When we had gone back to that other person I mentioned, she's been evaluated three times now. I can see that it's paid off. And she did well this year. She did really well."

One of the challenges for balancing both evaluation and support Garry identifies is time, "It's juggling the time." But he also recognizes that the evaluation is "necessary, it's needed, and I think overall it's a good system." He says that he is always working on evaluation and on the culture of the school. "I mean technically you are always doing it, but it's more of the time. It's less paperwork now as they pulled that back and refined it. It's more palatable and less time consuming, but if you don't keep up with it, oof, you've really got to budget your time." And he is constantly working on the "culture of the building. Like you don't want to lose ground on other things, for this to happen. That's the other piece."

The overarching frustration is for Zachary is, "I think it would be worth it if we saw results from it. But I'm not feeling as if we are seeing results. I'm always frustrated because I feel the amount of time we put in, we're not getting the results." Zachary is trying to figure out ways to balance the evaluation and support and the other responsibilities of an administrator. But when asked how you balance the evaluation 
system with supporting teachers he comments, "You can't balance evaluation and support. You can't."

\section{Recommendations to Selves}

Because balancing evaluation and supervision is so taxing, it seems to prompt administrators to consider how to make things easier. One realization that some of the administrators mentioned was that they were fortunate to have good teachers in their building who wanted to do well and do the right things. It also made Zachary realize the importance of hiring right.

Alicia recognizes that she is lucky to have good teachers. "Luckily being ranked high in the state, we have a lot of great teachers here. So in that regard, if you are ranked that high, your teachers are doing a really nice job.” She also identifies that her teachers "have a decent amount of seniority that they wanted to be here and they chose to be here." This helps with the climate of the school because teachers are invested in the collective success of the school. With such great teachers her challenge is supporting all of her teachers. She states, "How do I help her so this is not a waste of her time? And so that was really the challenge."

In addition to the managerial tasks and educational leadership tasks of a school administrator, Zachary also recognizes, "what this whole evaluation system has done is make smart principals or thoughtful or reflective principals realize that you have to hire right." He recognizes "hiring is the most important thing you can do. The problem is we tend to do it in the spring when we're trying to close the building, or in the summer when we're trying to open the building. We're rushed." But, "I'm very lucky. I have a very good staff. So that gives me more flexibility." 


\section{Chapter Summary}

The crux of the study was to try to understand what administrators are doing now to balance the often-opposing roles of supporting teacher growth and evaluating teacher performance. The administrators interviewed identified that balancing these roles is a difficult task, but they also discussed the many ways they are trying to do just that. The overarching theme that came from all of their efforts was their attempts to humanize the evaluation. They took steps to reduce the stress associated with the evaluation, they sought alterative types of accountability, and they laid a firm foundation of positive and supportive climate and culture through which they were able to be direct and supportive of their teachers. Despite their best attempts to humanize the evaluation, they also felt restricted by the evaluation system and the tool itself. They were unable to commit the time necessary to the parts of the evaluation they felt would be more beneficial for themselves and their teachers. 


\section{Chapter 5: Discussion}

\section{Introduction}

This qualitative phenomenological study was designed to investigate how current Rhode Island administrators are managing the dual roles of supporting and encouraging teacher growth and development, while also being responsible for evaluating teachers using the current RIDE high stakes teacher evaluation system. Through open-ended interviews, administrators discussed the type of climate and culture they try to establish at their schools, as well as the benefits and challenges they face implementing the RIDE evaluation system. They also discussed how the evaluation system has hampered or reinforced their ability to support teachers' professional growth. Administrators also addressed the various ways in which they have attempted to balance teacher growth and support with the evaluation system.

In this final chapter, I will summarize key findings from the administrator interviews. I will then address the research questions (balancing support and evaluation, supporting teacher growth, challenges and supports of evaluation system). I will then summarize those findings through the lens of all of the research questions, focusing on administrators' commonality of experiences. Lastly, I will discuss implications and limitations of these findings, as well as make recommendations for future research.

\section{Summary of Findings}

The administrators I interviewed are trying to find ways within their existing systems to merge or dovetail the RIDE evaluation with differentiated and relevant support for teachers. They identified the importance of accountability and recognized that some elements of the evaluation system are positive and helpful, while others are not 
beneficial. All discussed the importance of laying a foundation of a supportive and trusting climate in their schools. They noted that the foundation of a climate of trust assisted them with the implementation of the evaluation system. Yet, even with a strong culture, they still struggled with the high stakes nature of the evaluation system. When asked how they balance evaluation and supervision for their teachers, the frustrated response that came back was quite frankly, "we can't."

Despite the challenges administrators faced trying to balance evaluating and supporting their teachers, they all attempted to find ways to make it work. The administrators interviewed were not focused on the end results of the evaluation tool or even the specific supports they provided for their teachers. Rather, their goal was to positively impact teaching practices in their teachers' classes. Through the analysis of their interviews, I found there was a push and pull of accountability and support with an underlying foundation of a trusting culture. It was also important to retain a human element in the evaluation. In the next sections, I will discuss the important themes related to some of the challenges administrators faced, and how they attempted to balance their competing roles of evaluating and supporting their teachers. In each section I will first discuss the challenge identified, then the ways in which the administrators attempted to mediate that challenge, or research that addresses those challenges.

\section{Challenge \#1: Manipulation of Data}

When the RIDE evaluation system was initially introduced, it was proposed that teacher certification would be tied to evaluation ratings and any teacher who was rated ineffective for two years in a row would lose their certification. Additionally through the RTTT grant application, retention and promotion policies for teachers were also supposed 
to be connected with evaluation ratings (RIDE, 2010). While these components of the evaluation system were never put into place, there was a level of unease about the evaluation system before it was introduced and as it was rolled out (Borg, 2013b).

Administrators reported that teachers set SLO targets that were easily attainable, taught directly to their SLO targets, or manipulated the testing data to ensure the highest rating on their SLOs. Administrators also noticed that teachers were highly conscious of the scores they needed to ensure an effective or highly effective rating on all components of their evaluation. Administrators identified that these kinds of manipulations made it extremely difficult for them to rate teachers accurately using the evaluation tool. Because aspects of the evaluation system, like SLOs, were manipulated, administrators felt that many of the final effectiveness scores they gave were inflated. Administrator concerns were echoed by Linda Borg, education reporter for the Providence Journal, who stated that over two-thirds of RI principals using the RIDE Educator Evaluation system recognized that teachers were getting higher ratings then they felt were accurate. Borg also noted that over ninety-five percent of teachers were identified as effective or highly effective by their principals. The article states that over two-thirds of principals recognized that teachers were getting higher ratings then they felt were accurate. She criticized the system as being problematic, and identified these high ratings as "grade inflation" (Borg, 2013b).

Concerns of "grade inflation" are not without merit. Social science research has shown that as something becomes more high stakes, it is more likely that it will be subject to corruption. Berliner \& Nichols (2005) linked data corruption with the increase of high-stakes testing, identifying that in order to ensure higher scores, teachers would 
teach to the test, low-performing students would be excluded from testing, and administrators and teachers would misrepresent data. They also linked test corruption to teacher evaluation and teacher incentives, noting that if a teacher's professional career and reputation rested on the results of a given test or measure, it would be more challenging it will be to interpret the meaning of those test scores fairly and accurately (Berliner \& Nichols, 2005). When teachers were faced with a high stakes evaluation that was linked to their certification, and in some schools seniority, they were more likely to attempt to manipulate data or shift their focus toward achieving a specific rating or obtaining a certain score, rather than focusing on student achievement or on their own professional growth.

\section{Solution \#1: Social Learning and Peer Accountability}

To hold teachers more accountable and increase teacher engagement and ownership throughout the evaluation process, administrators created Professional Learning Communities and focus groups for teachers to identify topics of interest to them. They encouraged teachers to work together to craft SLOs and PGGs that addressed specific areas of need. Within the RIDE evaluation, SLOs are static documents in which teachers engage by themselves, or possibly with their evaluator. By developing a culture in which teachers work together to identify areas of need and evaluate them together, SLOs become living documents in which groups of teachers are engaging together to address real world problems or issues they are facing. Additionally, recognizing the importance of teachers learning from each other, several of the administrators spent time focusing on teachers sharing out ideas from some of their lessons, as well as sharing 
information from different PD workshops and sessions they attended both at the school and on their own.

In these ways, administrators used the tenets of social learning theory, which emphasizes the importance of learning together in a community (Dewey, 1916). Teachers are more likely to share ideas with each other and therefore learn from each other when they are provided with structured opportunities to work together (Garet, et al., 2001; Gossman, 2008). By taking into account the idea that teachers, as adult learners, are motivated to learn when facing a challenging situation or problem that arise in their work, and that it is important for them to have choice in what they are learning, who they are working with, and what they are studying (Knowles, 1973), administrators can provide teachers the framework within which they can impact their own professional growth and student achievement. Allowing teachers to identify and choose the type of PD they engage in, is more likely to benefit their professional practice (Borko, et al., 2002). Encouraging teachers to set goals together with their peers focused on student learning and achievement develops a sense of collective responsibility and (Garet, et al. 2001). Using Professional Learning Communities (PLCs) or focus groups, teachers are allowed to chose topics of interest to them, or identify topics they want to explore and learn about. By providing the structure for teachers to work together, administrators are encouraging teachers to create communities of support, collaboration, mentoring, and collective responsibility (Printy, 2008). This helps teachers to hold each other accountable as well as work collaboratively to develop as professionals. 
In this study, administrators encouraged teachers to work together by team, grade level, or department to identify concerns, develop goals, review achievement toward those goals together, and redefine what needs to happen based on progress being made toward the goals. Although this was a potential solution to helping teachers' craft genuine SLOs or PGGs, administrators noted a challenge to this mechanism for buttressing the evaluation system. Specifically, when the evaluation became cyclical and teachers were not all evaluated in the same year, teachers were no longer working together on the various components of the system at the same time. Administrators noted that this made it difficult for them to fully harness the power of social learning.

\section{Challenge \#2: One Size Does Not Fit All}

With the exception of evaluation frequency, there is no room for differentiation within the RIDE system. Although RIDE set the evaluation cycle for teachers based on their effectiveness rating from the previous year, it did not differentiate the components or expectations of the evaluation itself. Expert teachers are treated the same as novice teachers (Black, 2004) and veteran teachers often find evaluation humiliating because it does not recognize everything that a veteran teacher does when providing instruction in their class (Starratt, 1992).

Adult learning theory states that all teachers develop from novice to expert throughout their career, and that developmental level may change depending on the domain or new idea presented (Dreyfus, 2004). An expert teacher in one area will still need to progress through the stages again when a new concept is being taught, but may do so at a quicker pace than a novice teacher and may need different supports. All teachers need support and guidance as they introduce new concepts into their classroom 
practice, but these supports might look different for different teachers, as each individual will need varying amounts of time and support to move through the stages from novice to expert again (Berliner, 2001).

Expert teachers tend to want to do everything right, so they commit time and energy to the evaluation process that takes away from time they could be focusing on other areas, including sharing their expertise with other teachers and being a resource throughout the school for teachers and administrators alike. Because teachers learn and grow differently and at their own pace, their supports and evaluation should reflect that (Clarke \& Hollingsworth, 2002). With the current evaluation system, administrators do not have the opportunity to differentiate evaluation component requirements for an expert teacher who might need less formalized supports. Conversely, administrators are constrained by the evaluation system and are unable to provide new and struggling teachers with the additional supports that they need.

The administrators in this study expressed interest in developing some level of differentiation in the components of the teacher evaluation. They identified that they would like to spend more time with beginning or struggling teachers and allow more expert teachers the autonomy to do what they needed to do. Administrators also struggled with the challenge of making the evaluation process valuable and impactful for their most highly effective teachers.

\section{Solution \#2(a): Differentiation}

Although there was limited differentiation in the components of the evaluation, administrators interviewed differentiated the PD they offered to their teachers. Because of the evaluation, administrators were in their teachers' classes more frequently, and they 
were better able to identify where teachers were developmentally and provide them with the appropriate, necessary supports (Peno \& Silva Mangiante, 2012). They were able to identify places teachers might need additional support, as well as recognize ways an individual teacher could provide support to other teachers. Many of them used some type of choice in PD activities, ranging from a Bingo card, to playlists, to activities varied based on a pre-assessment of teachers' skills.

Research suggests that all teachers need the support of their colleagues, with whom they can share ideas (Duckworth, 2006). However, that support should look different for teachers at various stages of development. Peno and Silva Mangiante, (2012) created a model to provide mentoring support that is both purposeful and intentional. Their model takes advantage of Vygotsky's Zone of Proximal Development (1978) and intentionally pairs teachers with mentors who can provide them with the scaffolding necessary to help them grow. When administrators understand teachers' developmental level and are given flexibility to differentiate evaluation components, they are better able to support teachers and provide them with purposeful support.

\section{Solution 2(b): Tiered Evaluation}

Although administrators wanted to differentiate based on teacher needs, the RIDE system itself did not incorporate differentiation. Administrators struggled with working inside the parameters provided by the evaluation system and looked for ways to differentiate evaluation components and expectations for teachers based on developmental level and identified areas of need.

There are models of supervision that suggest a tiered approach to evaluation. Black (2004) proposed a tiered system that included tiers for beginning teachers, 
experienced teachers, and struggling teachers with some variation of expectations for teachers on the different tiers. Similarly, Glatthorn (1997) proposed a differentiated system including an intensive model for non-tenured teachers or those who need an improvement plan, a cooperative model for teachers working together, and a self-directed model where teachers set and work towards their own identified goals. Berube \& Dexter's suggestion (2006) includes five different levels of support, including (a) clinical, where teachers and administrators work together through clinical supervision, (b) collegial, where teachers are supporting each other, (c) self-directed, where teachers set and work on their own goals individually, (d) informal, where administrators conduct frequent observations and engage with teachers in discussions about their teaching practice, and (e) inquiry based, where teachers are asking a specific question and work on answering that question.

\section{Challenge \#3: Negativity Toward Evaluation}

Administrators reported that their teachers viewed the evaluation system as punitive and cumbersome. When the educator evaluation system was first designed and developed, RIDE proposed tying certification to it, including providing financial incentives for teachers, as well as dismissing teachers who were rated ineffective for two years in a row (Rhode Island Department of Education, 2010). None of these were ever

established, but having them included in the initial RTTT grant, created apprehension and mistrust in the teachers from the beginning. This fear of the evaluation system being punitive was recognized shortly after implementation. Again, Linda Borg (2013a) discussed the issue in the Providence Journal, citing that teachers feared losing their certification, that they believed the evaluation system was designed to punish them, and 
that they considered Student Learning Objectives as unfair measures of student achievement and their teaching ability.

Evaluation systems perpetuate a hierarchical relationship between teachers and administrators (McBride \& Skau, 1995; Murphy, et al., 2013). This counteracts the development of a positive climate and culture of the school. Research emphasizes the importance of creating a trusting environment and empowering teachers as a way of positively impacting teacher growth and development (Colton \& Sparkes-Langer, 1993; Donaldson, 2013; Starratt, 1992; Sullivan \& Glanz, 2000). While administrators may attempt to focus on the development of a positive culture, evaluation systems are authoritative and shift the focus to identifying teachers' weaknesses, can therefore shut down teacher growth (McBride \& Skau, 1995). Continuing to perpetuate the hierarchical nature developed through evaluations, rather than focusing on supporting teachers and building a culture of collaboration, will show little benefit to teachers' performance in the classroom (Murphy, et al., 2013).

Administrators in this study echoed concerns about teachers shutting down. They noted that teachers felt ashamed or shutdown when they received scores that were not in the effective or highly effective range. Teachers often focused on scores and ratings while not hearing the positive and/or constructive feedback that administrators had to offer. Administrators struggled with ways in which they could conduct the evaluations fairly and provide teachers with honest feedback, while maintaining the positive culture they worked so hard at developing. 


\section{Solution \#3: Positive Culture and Building Trust}

Administrators in this study attempted to address teachers' concerns of highstakes evaluation by supporting teachers through ongoing professional development around the evaluation components, which is recognized as good practice when implementing an evaluation (Goe, et al., 2008). The administrators also attempted to negate some of the teachers' apprehensions by reducing paperwork related to the evaluation, simplifying the process as much as possible, conducting group meetings for all teachers, and using already established systems in their schools as ways to collect data. Administrators attempted to reassure teachers that they were using evaluation in a way that was supportive, and were not using it as a "gotcha" system.

To buttress the power differential inherent in the evaluation system, administrators in this study also focused on developing a positive climate and culture in their schools. They tried to develop an environment of trust, a sense of teacher empowerment, and a shared sense of collective responsibility.

They spent a lot of time building trust with their teachers, encouraging them to take risks, and supporting them when they failed. They provided support not only in pedagogy, but also in the evaluation tool itself. Therefore, they found many teachers willing to put in effort with the evaluation process and new expectations related to classroom practice, because they were comfortable taking risks and trusted their administrators.

Some administrators tried to continue to develop trust throughout the evaluation process by dividing up responsibilities for the evaluation, having one administer evaluate, while another one would be responsible for providing support. They also provided 
additional supports for teachers such as group and individual trainings on the various components of the evaluation, clearly identifying and defining expectations, offering teachers a do-over for a less-than-successful observation, and conducting as many additional observations as necessary. In addition, administrators worked hard at building relationships with their teachers. They recognized the importance of being present throughout the school and in their teachers' classrooms. These supports helped some of the teachers view the evaluation as a way to grow, rather than a punishment.

The importance of building a positive climate in schools cannot be over emphasized. Developing a collaborative culture where teachers take collective responsibility for the overall success of the students at the school is hard work, but the administrators I interviewed recognized that it is well worth the effort. When administrators take the time to focus on developing a shared culture of professionalism, they are more likely to see an impact on teacher professional growth (Donaldson, 2013; McBride \& Skau, 1995; Starratt, 1992).

The importance of building a trusting relationship with their teachers is echoed in the literature on providing quality support and supervision (Colton \& Sparkes-Langer, 1993; McBride \& Skau, 1995; Sullivan \& Glanz, 2000). Trust is seen as the foundation for support in schools. When trust is present teachers are more likely to take risks and try new strategies. They are also more likely to work together and share ideas and problems because they identify the culture of one that is working together as members of a singlecommunity (McBride \& Skau, 1995). The development of a shared sense of collective responsibility is an essential factor in developing teacher self-efficacy and holding teachers accountable for what happens in their classrooms (Printy, 2008). 


\section{Limitations of the Study}

While findings from this study offer insights into how some administrators balance the roles of supervision and evaluation, the study also has limitations. First this study involved a small number of participants. While the six administrators interviewed were representative of all grade levels, there is limited information that can be gathered from only six interviewees. Second, all of the interviewees were from similar, suburban, public schools throughout Rhode Island. Third, those individuals who agreed to be interviewed were interested in making evaluation and supervision work for their teachers. Therefore, they might not be representative of all administrators. Additionally, several participants were involved in graduate work. Their willingness to be involved in research might have prompted them to identify with the benefits of participating in research, as well as the challenges of conducting research. These factors also suggest that the participants in this study may represent a unique subsample of administrators. Fourth, two administrators, at their request, were interviewed together. As a principal and assistant principal pair, they answered questions together often adding to each other's answers. Although they provided detailed information, I cannot know if they would have said something different if they were interviewed separately. Lastly, although I asked for artifacts, not many were provided which meant there was limited data to be used for triangulation. Additionally, although attempts were made to involve the administrators in member checking, only one responded.

\section{Implications}

Although this phenomenological view of administrators attempting to balance evaluation and support of teacher growth is a small sample and cannot be generalized, the 
experiences of these administrators may be helpful to others faced with similar challenges or those who might be considering developing or revising an evaluation system.

The administrators in this study clearly identified the importance of developing a strong, positive, trusting environment in their schools. They recognized that having a trusting environment helped them to implement various new initiatives including the evaluation. Even given the struggles they faced with negativity and the limitations of the evaluation, they recognized that without the supportive culture they have developed, it would be even more difficult. Because of the culture, they were able to increase teacher buy-in to the process. As adult learners, it is important for teachers to feel valued and appreciated. They need to be active learners and participants in the process for change to happen.

Just as developing a strong culture takes work, providing appropriate levels of supports for all teachers also takes work. When attempting to marry an evaluation system with such supports, it is important for administrators to have a high level of flexibility to tailor programs to meet the needs of individual teachers, groups of teachers, and the overall school needs and goals. This flexibility needs to come in the form of carefully selecting the evaluation components that work best for individual teachers, as well as creating professional development opportunities to meet the needs of teachers at various stages of development.

Above all else, the administrators in this study recognized the importance of the human element. They understand that hard situations will occur and it is how you handle them that make all the difference. When you approach your teachers through the lens of a positive, trusting environment and the presumption that they are there to do a good job 
and do their best for kids, then you are starting from a place of positivity rather than negativity. "When you go in through that light teachers can hear that more. Hardly a teacher gets to work and says I really want to do a bad job today. Let me get up so I cannot help kids learn." (Alicia).

Administrators used words such as marry, merge, and dovetail to describe the ways in which they attempted to balance evaluation and supervision. But they also used phrases like, "we can ' $t$ " and "we don' $t$ " when discussing their ability to balance evaluation and support. Even given the myriad of challenges administrators faced, they tried their best to make it all work. They laid a solid foundation of a positive and trusting culture at their schools and then delicately balanced the accountability of the evaluation with the supports of professional development provided for their teachers to help make all students successful.

\section{Suggestions for Future Research}

This study gave us insight into the experiences of six administrators who are attempting to balance evaluation and supervision for their teachers. Future research could investigate how additional principals attempt to balance these roles as well. Using the data collected in this research, a survey could be developed to use with a larger sample of administrators to collect a broader view of administrators' experiences. Another area that this research could be expanded is by asking teachers, either through interviews or surveys, about their experiences related to evaluation to investigate how they are similar to, or differ from, those of administrators. 


\section{Appendix A}

\section{Participant Email}

Dear Rhode Island Principal,

My name is Mary Slattery. I have been the principal at Exeter-West Greenwich Junior High School for the past six years. I am contacting you because I believe you are a principal in a Rhode Island school currently using the RIDE Educator Evaluation tool who is also responsible for supporting your teachers' growth. I would like to invite you to participate in my research study.

I am a Ph.D. in Education candidate at the University of Rhode Island/Rhode Island College. My dissertation research seeks to document the experiences of Rhode Island principals and explore how they balance the dual roles of evaluating and supporting their teachers using the RIDE Educator Evaluation tool. This research has been approved by the University of Rhode Island (URI) and the Institutional Review Board (IRB).

I am looking for principals who have been at their current schools for a minimum of 2 years, who are using the RI educator evaluation system and are responsible for both supporting and evaluating their teachers.

The research will consist of one in-depth interview about your experiences evaluating your teachers using the RI Educator Evaluation tool as well as supporting your teachers professional growth and development. The interview will last approximately 60 minutes. I will be sharing a verbatim transcript and my analysis with you via email to ensure that I have understood what you told me and am accurately portraying your experiences. A follow up interview might be necessary for clarification purposes. Interviews will be held face to face at a location and time of your choice. Total time required for participation is a 60-minute interview and possibly a 15-minute follow up interview if deemed necessary for clarification purposes.

I will also be asking you to provide documents and other examples of ways you have both supported and evaluated your teachers. Sample documents could include, but are not limited to, professional development calendars, activities, notes, outlines or presentations; common planning time schedules, agendas, and/or minutes; other documents that demonstrate your role supporting your teachers; and evaluation documents such as notes from observations, conferences, feedback provided and other documents that demonstrate your role evaluating your teachers

Your part in this study is confidential and voluntary. None of the information will identify you, your school, or your district by name. Pseudonyms will be used in any written analysis.

If you are interested in participating, please complete this questionnaire. Feel free to call me if you would like more information. I look forward to talking to you. 
Best,

Mary Slattery

(401) 569-9584

mfslattery03@gmail.com 


\section{Appendix B}

\section{Participant Questionnaire (Adapted from Google Form)}

\section{Study Questionnaire}

This form is to determine eligibility and obtain contact information for my research study on the roles of principals evaluating and supervising teachers.

1: Name: First and last

2: Phone Number

3: Email address

4: Mailing address: To be used for mailing consent form for research study.

5: School district

6: School name

7: Grades at your school: Check all that apply

Kindergarten

Grade 1

Grade 2

Grade 3

Grade 4

Grade 5

Grade 6

Grade 7

Grade 8

Grade 9

Grade 10

Grade 11

Grade 12

Other

8: By submitting this survey you are consenting to allowing the researcher, Mary Slattery, to contact you in regards to this survey.

Mark only one:

Yes, I consent to further contact regarding this research study.

No, I DO NOT consent to further contact regarding this study.

(If no, end survey.) 
9: Have you been at your current school for at least two years?

Mark only one.

Yes

No

(If no, go to section of form for those not qualifying for interview.)

Qualifications

10: Do you use the RI educator evaluation system at your school?

Mark only one.

Yes

No

(If no, go to section of form for those not qualifying for interview.)

11: Are you responsible for both evaluating teachers at your school and supporting their growth and development?

Mark only one.

Yes

No

(If no, go to section of form for those not qualifying for interview.)

Evaluation System

12: Who implements the evaluation system at your school?

Check all that apply.

Principal

Assistant Principal

Department Chair

Team Leader

Grade Level Leader

Other

Thank you

(For those qualifying for interview)

Thank you for completing this survey. I will be contacting you shortly to set up time for an Interview. If you have any questions feel free to contact me at

MFSlattery03@gmail.com

(For those not qualifying for interview)

Thank you for completing this survey. I am seeking principals that are currently using the RI educator evaluation system, have been at the same school for at least two years, and who both support and evaluate teachers at their school.

If you have any questions feel free to contact me at MFSlattery03@gmail.com 


\section{Appendix C}

\section{Informed Consent Form}

The University of Rhode Island

Department of: Education

Address: Kingston, RI, 02881

"How Principals Balance Dual Roles of Evaluation and Support"

\section{CONSENT FORM FOR RESEARCH}

You have been invited to take part in a research project described below. The researcher will explain the project to you in detail. You should feel free to ask questions. If you have more questions later, Dr. Theresa Deeney, the primary investigator responsible for this study, can be contacted via phone at (401)874-2682 or email tdeeney@uri.edu or primary researcher Mary F. Slattery, at (401) 569-9584 or mfslattery03@gmail.com.

\section{Description of the project:}

Recent reforms have focused on the importance of developing and implementing educator evaluation tools as a way of measuring effective teachers. Teacher evaluation systems are designed to identify effective teachers but are not intended to support teacher development or assist "less than effective" teachers to improve their classroom practices. The job of both evaluating and supporting teachers falls largely to the principal. Through the use of open-ended interviews, this research seeks to explore, study and report out the experiences of Rhode Island principals. The goal of this research is to share the experiences of principals and the challenges they are facing in balancing the dual roles of supporting and evaluating their teachers.

What will be done:

If you decide to take part in this study here is what will happen: You will participate in one 60-minute interview conducted by the researcher. The interviews will be audio taped and later transcribed and analyzed for themes. The transcription and analysis will be shared with you within two weeks so you can check the accuracy of what you said as well as accuracy of analysis.

Risks or discomfort:

I do not anticipate any risks to you by participating in this study.

Benefits of this study:

The only benefit that you may receive by participating in this study is in having your opinion and experiences reported and used to understand the challenges faced by Rhode Island principals in balancing evaluating and supporting teachers in their schools. 


\section{Confidentiality:}

Your part in this study is confidential. None of the information shared will identify you, your school, or district by name.

Decision to quit at any time:

The decision to take part in this study is up to you. You do not have to participate. If you initially decide to take part in the study, you may quit at any time. Whatever you decide will in no way penalize you. If you wish to quit, simply inform Mary Slattery (401) 569-9584 of your decision.

\section{Rights and Complaints:}

If you are not satisfied with the way this study is performed, you may discuss your complaints with Mary Slattery or with Dr. Theresa Deeney via phone (401) 874-2682 or email tdeeney@uri.edu, anonymously, if you choose. In addition, if you have questions about your rights as a research participant, you may contact the office of the Vice President for Research and Economic Development, 70 Lower College Road, Suite 2, University of Rhode Island, Kingston, Rhode Island, telephone: (401) 874-4328.

Your signature on this form means that you understand the information and you agree to participate in this study. You have read the Consent Form. Your questions have been answered.

Signature of Participant

Typed/printed Name

Date
Signature of Researcher

Typed/printed name

Date

You consent to have your interview audiotaped.

Signature of Participant

Typed/printed Name
Signature of Researcher

Typed/printed name

Date

Date

Please sign both consent forms, keeping one for yourself. 


\section{Appendix D}

\section{Interview Protocol}

\section{SUPPORT}

- Describe what professional development looks like at your school.

- What is your role in professional development in your school?

○ Whole school

- Groups

$\circ \quad$ Individual

- Tell me about the types of supports, if any, you provide for teachers.

○ Formal supports

- Informal supports

- How do you differentiate supports for teachers at different levels?

- Novice teachers

- Effective or highly effective teachers

- Ineffective or developing teachers

\section{EVALUATION}

- Tell me about the evaluation system in your school

- How do teachers in your school set professional growth goals for the evaluation system?

- Whole school/groups/ individual?

- Who decides?

- Describe some of the successes, if any, with the evaluation program. 
- Describe some of the challenges, if any, with the evaluation program.

\section{CONFLICT}

- Describe any positive impact the evaluation system has on your ability to support your teachers:

- Novice teachers

- Effective or highly effective teachers

- Ineffective or developing teachers

- Provide example(s)

- Describe the negative impact the evaluation system has on your ability to support your teachers:

- Novice teachers

- Effective or highly effective teachers

- Ineffective or developing teachers

- Provide example(s)

- Describe how the evaluation system has changed the way in which your support teachers

- Novice teachers

- Effective or highly effective teachers

- Ineffective or developing teachers

\section{BALANCING}

- Describe any challenges you have faced balancing the dual roles of evaluator and supporter of your teachers. 
- Describe any benefits you have experiences balancing the dual roles of evaluator and support of your teachers.

\section{CONCLUSION}

- What other information would you like to add?

- Do you have any further comments or questions? 


\section{Bibliography}

Allen, R., \& Casbergue, R. (1997). Evolution of Novice Through Expert Teachers' Recall: Implications for Effective Reflection on Practice. Teaching and teacher Education , 13 (7), 741-755.

Bambrick-Santoyo, P. (2013, October). Rookie Teachers Need Dress Rehearsals Too. Kappan , 72-73.

Berliner, D. (2001). Learning About Learning from Expert Teachers. International Journal of Educational Research, 35, 463-482.

Berliner, D., \& Nichols, S. (2005). The Inevitable Corruption of Indicators and Educators Through High-Stakes Testing. Tempe, AZ: Education Policy Studies Laboratory.

Berube, B., \& Dexter, R. (2006). Supervision, Evaluation and NCLB: Maintaining a Most Highly Qualified Staff. Catalyst for Change , 34 (2), 11-17.

Black, S. (2004, December). Helping Teachers Helps Keep Them Around. American School Board Journal , 46-51.

Borg, L. (2013b, October 11). High Evaluation Ratings for Most R.I. Teachers Problematic. Providence journal .

Borg, L. (2013a, September 13). Study: R.I. Teacher Evaluation Seen as Punitive. Providence Journal . Providence, RI.

Borko, H., Elliot, R., \& Uchiyama, K. (2002). Professional Development: A Key to Kentucky's Educational Reform Effort. Teaching and Teacher Education, 18, 969-987.

Brophy, J. (1986, October). Teacher Influences on Student Achievement. American Psychologist, 1069-1077.

Campbell, R. J., Kyriakides, L., \& Muijs, R. D. (2003 ). Differential Teacher Effectivness: Towards a Model for search and Teacher Appraisal. Oxford Review of Education , 29 (3), 347-362.

Clandinin, D., \& Connely, F. (2000). Narrative Inquiry: Experience and Story in Qualitative Research. San Francisco, CA: John Wiley \& Sons, Inc. 
Clarke, D., \& Hollingsworth, H. (2002). Elaborating a Model of Teacher Professional Growth. Teaching and Teaacher Education , 18, 947-967.

Cleary, M., \& Groer, S. (1994). Inflight Decisions of Expert and Novice Healthy Teachers. Journal of School Health , 64 (3), 110-114.

Colton, A., \& Sparkes-Langer, G. (1993). A Conceptual Framework to Guide the Development of Teacher Reflection and Decision Making. Journal of Teacher Education , 44 (1), 45-54.

Creswell, J. (2009). Research Design. Los Angeles, CA: Sage Publications.

Creswell, J., \& Miller, D. (2000). Determining Validity in Qualitative Inquiry. Theory Into Practice , 39 (3), 124-130.

Daley, B. (1999). Novice to Expert: An Exploration of how Professionals Learn. Adult Educaiton Quarterly , 49 (4), 148-163.

Darling-Hammond, L. (2000). Teacher Quality and Student Achievement: A Review of State Policy Evidence. Education Policy Analysis Archives , 8 (1), 1-44.

Darling-Hammond, L., Amrein-Beardsley, A., Haertel, E., \& Rothstein, J. (2012). Evaluating Teacher Evaluation. Phi Delta Kappan, 93 (6), 8-15.

Dewey, J. (1916). Democracy and Education: An Introduction to the Philosophy of Education. Lexington, KY: BLN Publishing.

Ding, C., \& Sherman, H. (2006). Teaching Effectiveness and Student Achievement: Examining the Relationship. Educational Reserach Quarterly, 29 (4), 40-49.

Donaldson, M. (2013). Principals' Approaches to Cultivating Teacher Effectiveness: Constraints and Opportunities in Hiring, Assigning, Evaluating and Developing Teachers. Educational Administration Quarterly , 49 (5), 838-882.

Dreyfus, S. (2004). The Five-Stage Model of Adult Skill Acquisition. Bulletin of Science, Technology \& Society, 24 (3), 177-181.

Duckworth, E. (2006). The Having of Wonderful Ideas. In E. Duckworth, "The Having of Wonderful Ideas" \& Other Essays on Teaching and Learning (pp. 1-14). Teachers College Press.

Evans, J. (2002). Effective Teachers: An Investigation from the Perspective of Elementary School Students. Action in Teacher Education , 24 (3), 51-62.

Fendler, L. (2003). Teacher Reflection in a Hall of Mirrors: Historical Influences and Political Reverberations. Educational Researcher, 32 (3), 16-25. 
Ferry, N. M., \& Ross-Gordon, J. M. (1998). An Inquiry into Schon's Epistemology of Practice: Exploring Links Between Experiene and Relfective Practice. Adult Education Quarterly , 48 (2), 98-112.

Fraenkel, J., Wallen, N., \& Hyun, H. (1993). How to design and evaluate research in education. New York, NY: McGraw Hill.

Garet, M., Porter, A., Desimone, L., Birman, B., \& Yoon, K. (2001). What Makes Professional Development Effective? Results from a National Sample of Teachers. American Educational Research Journal , 38 (4), 915-945.

Geerinck, I., Masschelein, J., \& Simons, M. (2010, April 23). Teaching and Knowledge: A Necessary Combination? An Elaboration of Forms of Teachers' Reflexivity. Studies in Philosophy \& Education, 379-393.

Glatthorn, A. (1997). Differentiated Supervision. Alexandria, VA: Assocation for Supervision and Curriculum Development.

Goe, L., Bell, C., \& Little, O. (2008). Approaches to Evaluating Teacher Effectiveness: A Research Synthesis. National Comprehensive Center for Teacher Quality .

Gossman, P. (2008, Spring). Teaching Development - Experience and Philosophy (Using the Three Rs). Teacher Education Quarterly, 155-169.

Gurskey, T. (1986, May). Staff Development and the Process of Teacher Change. Educational Researcher, 5-12.

Hazi, H., \& Rucinski, D. (2009). Teacher Evaluation as a Policy Target for Improved Student Learning: A Fifty-State Review of Statute and Regulatory Action since NCLB. Education Policy Analysis Archives , 17 (5), 1-18.

Hofer, B. K., \& Pintrich, P. R. (1997). The Development of Epistemological Theories: Beliefs About Knowledge and Knowing and Their Relation to Learning. Review of Educational Reserach , 67 (1), 88-140.

Jaeger, E. (2013). Teacher Reflection: Supports, Barriers, and Results. Issues in Teacher Education , 22 (1), 89-104.

Jones, M. G., \& Vesilind, E. M. (1996). Putting practice into theory: Changes in the organization of preservice teachers' pedagogical knowledge. American Educational Research Journal , 33 (1), 91-117.

Kim, Y., \& Baylor, A. (2006). A Social-Cognitive Framework for Pedagogical Agents as Learning Companions. Association for Educational Communications and Technology , 54 (6), 569-596. 
King, P. M., \& Kitchener, K. S. (2004). Reflective Judgment: Theory and Reserach on the Development of Epistemic Assumptions Through Adulthood. Educational Psychologist, 39 (1), 5-18.

Knowles, M. (1973). The Adult Learner: A Neglected Species. Houston, TX: Gulf Publishing Company.

Marshall, K. (2012, November). Fine-Tuning Teacher Evaluation. Educational Leadership, 50-53.

Marzano, R. (2012, November). The Two Purposes of Teacher Evaluation. Educational Leadership , 14-19.

McBride, M., \& Skau, K. (1995). Trust, Empowerment, and Reflection: Essentials of Supervision. Journal of Curriculum and Supervision , 10 (3), 262-277.

Merriam, S. (2009). Qualitative Research: A Guide to Design and Implementation. San Francisco, CA: Jossey-Bass.

Mertler, C. (2016). Introduction to Educational Research. Thousand Oaks, CA: Sage Publications.

Moir, E. (2010, Winter). Reflections. Retrieved Nov 22, 2015, from New Teacher Center: http://www.newteachercenter.org/node/1700

Moustakas, C. (1994). Phenomenological Research Methods. Thousand Oaks, CA: Sage Publications.

Munby, H., Russell, T., \& Martin, A. (1997). Teachers' Knowledge and How It Develops. In V. Richardson, Handbook of Research on Teaching (pp. 877-901). New York, NY: Macmillan.

Murphy, J., Hallinger, P., \& Heck, R. (2013). Leading via Teacher Evaluation: The Case of the Missing Clothes? Educational Researcher , 42 (6), 349-354.

National Assessment of Educational Progress (NAEP). (2014). The Nation's Report Card. Retrieved October 9, 2016, from http://www.nationsreportcard.gov/

National Commission on Excellence in Education. (1983, April). A Nation At Risk: Archived Information. Retrieved July 18, 2014, from U.S. Department of Eduation: http://www2ed.gov/pubs/NatAtRisk/risk/html

Patton, M. (2002). Qualitative Research \& Evaluation Methods. Thousand Oaks, CA: Sage Publications, Inc. 
Peno, K., \& Silva Mangiante, E. (2012). The Journey from Novice to Expert: Toward a Purposeful On-going Mentoring Model. In C. Boden, \& K. King, Conversations about Adult Learning in a Complex World. Charlotte, NC: Information Age Publishing.

Printy, S. (2008). Leadership for Teacher Learning: A Community of Practice Perspective. Educational Administrative Quarterly , 44 (2), 187-221.

Rhode Island Department of Education. (2010, May 28). Rhode Island Department of Education. Retrieved July 18, 2014, from Race to the Top Application for Initial Funding: http://media.ride.ri.gov/commissioner/RTTT/Combined-NarrativeFINAL-0527.pdf

Rhode Island Department of Education. (2012). Rhode Island Model: Teacher Evaluation \& Support System. Providence: RIDE.

Rhode Island Department of Education. (2011). RI Beginning Teacher Induction Program. Retrieved Nov 22, 2015, from Rhode Island Department of Education: http://ride.ri.gov/Portals/0/Uploads/Documents/Teachers-and-AdministratorsExcellent-Educators/Induction/Induction-Model-Final.pdf

Rivkin, S., Hanushek, E., \& Kain, J. (2005). Teacher, Schools, and Academic Achievement. Econometrica, 73 (2), 417-458.

Schon, D. (1987). Educating the Reflective Practitioner. San Francisco, CA: Jossey-Bass.

Segall. (2004). Revisiting Pedagogical Content Knowledge: The Pedagogy of Content/ the Content of Pedagogy . Teaching and Teacher Education, 20, 489-504.

Shulman, L. (1986). Those Who Understand: Knowledge Growth in Teaching. American Educational Research Association, 15 (2), 4-14.

Shulman, L., \& Sherin, M. (2004). Fostering Communities of Teachers as Learners: Disciplinary Perspectives. Journal of Curriculum Studies , 36 (2), 135-140.

Smith, M. (2003, January). Communiities of Practice. Retrieved November 28, 2014, from The Encyclopedia of Informal Education:

www.infed.org/biblio/communities_of_practice.htm

Starratt, R. (1992). Perspectives and Imperatives After Supervision. Journal of Curriculum and Supervision, 8 (1), 77-86.

Sullivan, S., \& Glanz, J. (2000). Alternative Approaches to Supervision: Cases from the Field. Journal of Curriculum and Supervision , 15 (3), 212-235. 
Tedesco, J. (1991). Women's Ways of Knowing/ Women's Ways of Composing. Rhetoric Review , 9 (2), 246-256.

U.S. Department of Education. (2001). NCLB Legislation Public Law PL 107-110, the No Child Left Behind Act of 2001: Retrieved July 18, 2014, from U.S. Department of Education: http://www2.ed.gov/policy/elsec/leg/esea02/pg2.html\#sec1119

U.S. Department of Education. (2013, June 7). Race to the Top Fund. Retrieved October 13, 2013, from U.S. Department of Education: http://www2.ed.gov/programs/racetothetop/index.html

U. S. Department of Education. (2001). U. S. Department of Education. Retrieved July 18, 2014, from NCLB Legislation Public Law PL 107-110, the No Child Left Behind Act of 2001: http://www2.ed.gov/policy/elsec/leg/esea02/pg2.html\#sec1119

Vygotsky, L. (1978). Mind in Society. Cambridge, MA: Harvard University Press.

Wise, A. E., Darling-Hammond, L., McLaughlin, M. W., \& Bernstein, H. T. (1985). Teacher Evaluation: A Study of Effective Practices. The Elementary School Journal , 86 (1), 60-121.

Wright, P., Horn, S., \& Sanders, W. (1997). Teacher and Classroom Context Effects on Student Achievement: Implications for Teacher Evaluation. Journal of Personnel Evaluation in Education , 11, 57-67. 